\title{
Space-, time- and spin-resolved photoemission
}

\author{
Gerd Schönhense $^{\mathrm{a}, *}$, Katerina Medjanik ${ }^{\mathrm{a}, \mathrm{b}}$, Hans-Joachim Elmers ${ }^{\mathrm{a}}$ \\ a Institut für Physik, Johannes Gutenberg-Universität, 55128 Mainz, Germany \\ b MAX IV Laboratory, Ole Römers vag 1, 22363 Lund, Sweden
}

\section{A R T I C L E I N F O}

\section{Article history:}

Available online 10 June 2015

\section{Keywords:}

Photoemission

Imaging

PEEM

Time resolved

Spin resolved

ToF momentum microscope

Spin filter

\begin{abstract}
A B S T R A C T
This article reviews photoemission experiments that simultaneously resolve at least two of the following degrees of freedom: space (real and momentum space), time (intrinsic time scale of a fast experiment or time-of-flight) and spin. In the spatiotemporal domain, imaging of fast processes by PEEM gives direct insight into plasmon dynamics or magnetization processes. In the category real space $\mathcal{E}$ spin the novel concept of imaging spin filters is discussed. In the time $\mathcal{E}$ spin chapter we address time-of-flight spin detectors and ultrafast spin processes that are accessible by pump-and-probe techniques. A main part of the paper is devoted to the resolution of momentum-space $\&$ time. This is implemented in form of the time-of-flight momentum microscope, a very recent development of which the first instrument has been in operation since 2014. In an extended outlook chapter, the potential of new developments and of a novel, highly parallelized delay-line type electron detector will be discussed. The combination of all three degrees of freedom $k$-space, time $\mathcal{E}$ 'spin is emerging through the combination of the ToF momentum microscope with imaging spin filter.
\end{abstract}

(c) 2015 Elsevier B.V. All rights reserved.

\section{Introduction}

Launched by pioneering work in Sweden [1], photoelectron spectroscopy (PES) has become a standard tool in many disciplines in physics, chemistry and materials research. After half a century of continuous development, we are still facing a strong need for further improvements of photoemission techniques. The situation is satisfactory in conventional intensity spectroscopy, where the

Abbreviations: 2PPE, two-photon photoemission; ARPES, angular resolved photoemission spectroscopy; BBO, beta-barium borate; BFP, backfocal plane; CCD, charge-coupled device; CDAD, circular dichroism in the angular distribution; CES, constant energy surface; DLD, delay-line detector; EUV, extreme ultraviolet radiation; FEL, free-electron laser; FIB, focused ion beam etching; FoM, figure of merit; FWHM, full width at half maximum; HA, hemispherical analyzer; HAXPES, hard $\mathrm{X}$-ray photoelectron spectroscopy; HHG, high-harmonics-generation; LDAD, linear dichroism in the angular distribution; LEED, low-energy electron diffraction; LEEM, low-energy electron microscopy; MCP, microchannel plate; MRAM, magnetic random access memory; PEEM, photoemission electron microscopy; PES, photoelectron spectroscopy; SBZ, surface Brillouin zone; SNOM, scanning near-field optical microscopy; SPP, surface plasmon polaritons; ToF, time-of-flight; ToFPEEM, time-of-flight photoemission electron microscopy; TR-PEEM, time-resolved photoemission electron microscopy; TR-XTM, time-resolved X-ray transmission microscopy; UHV, ultra-high vacuum; XMCD, X-ray magnetic circular dichroism; XPEEM, X-ray photoemission electron microscopy; XTM, X-ray transmission microscopy; XUV, extreme ultraviolet radiation.

* Corresponding author.

E-mail address: schoenhe@uni-mainz.de (G. Schönhense). high degree of maturity of energy analyzers manifests in impressive figures for energy and angular resolution (of the order of $1 \mathrm{meV}$ and $0.1^{\circ}$, respectively [2,3]). Owing to efficient $2 D$ data acquisition in the $E-\theta$ mode of operation with $\sim 10^{4}$ data points in parallel, state-of-the-art hemispherical analyzers are the workhorses of photoelectron spectroscopy. Throughout the decades we have seen substantial improvements, mainly with respect to better lens optics, magnetic shielding and detectors and to a lesser degree with respect to the analyzers themselves.

Fueled by tremendous advances in the brilliance, energy range and time structure of photon sources, developments are now emerging that go beyond the mainstream method of intensity spectroscopy in the $E-\theta$ mode. New possibilities for increased depth information come from high-brilliance synchrotron sources and FELs in the hard X-ray regime. At the same time, we are facing an increasing need for improved spatial and/or temporal resolution. Pulse lengths in the femtosecond range open the door to a new generation of experiments targeting ultrafast dynamics and short-lived transient states [4]. On the laboratory scale, numerous table-top devices for the production of ultrashort EUV or X-ray pulses [5] are being developed. In particular, free electron lasers (FELs) [6] have emerged as a fascinating research tool to study atoms, molecules and solids with a time resolution of a few femtoseconds and a spatial resolution down to the Å range. One key question in condensed matter physics that may be answered by these novel photoemission experiments is how complex and often 
unexpected phenomena emerge from the competing interactions in a quantum many-body system, leading to remarkable macroscopic phenomena such as high- $T_{C}$ superconductivity, colossal magnetoresistance and metal-insulator transitions. Time-resolved studies after controlled excitation with light pulses out of equilibrium offer the possibility to identify the most relevant relaxation channels and to potentially allow us to gain insight into the driving forces for unexpected ground state properties.

A third degree of freedom which is presently gaining importance is the photoelectron spin. Electron spin polarimetry is no longer the domain of surface magnetism, as the emergence of "Rashba physics" [7] has had a strong impact on spin-resolved PES, in particular in the context of topological insulators [8], with challenging potential applications. In this article we discuss experiments and technical developments and achievements that resolve at least two of these three degrees of freedom. The coordinate space can refer to real or to reciprocal space. The coordinate time can denote the intrinsic timescale of a fast experiment or can refer to a time-offlight (ToF) experiment where the time coordinate is measured and converted into an energy value.

Simultaneous resolution of real space $\mathcal{E}$ time is the domain of time-resolved photoemission electron microscopy (TR-PEEM). The study in this area was initiated by early work at synchrotron sources [9-12], where X-ray circular dichroism is exploited as a magnetic contrast mechanism, and using short-pulse lasers [13-17], where plasmonic structures, near fields and electrical polarization dynamics can be studied on a femtosecond timescale. Within a decade, this method became an established tool, accessible for a wide user community at synchrotrons and in numerous lab experiments worldwide. In Section 2, some selected achievements with synchrotron and laser excitation will be highlighted, demonstrating the state-of-the-art of TR-PEEM.

Spin detection $\mathcal{E}$ spatial resolution (in parallel-imaging mode) is the key feature of multichannel spin detection. This is an emerging technique, not yet commonly available. There are currently three cases of successful application, differing in the possible gain factors: Firstly implementation of a $2 D$ multichannel spin detector in the exit of a hemispherical analyzer exploits the $E-\theta$ mode of operation for spin analysis; parallel detection of the order of $10^{3}$ data points has been reached in a pilot experiment [18] and in the first commercial system [19]. This application is the most demanding of the three imaging spinfilter techniques, due to the large exit field, the energy spread along the dispersive direction and the strong astigmatism at the exit of the hemispherical analyzer. The present status of the ongoing development is reported in Section 3, while the prospects and obstacles are discussed in the outlook section.

A higher degree of parallelization can be reached by implementing a $2 D$ imaging spin filter into the column of an electron microscope as demonstrated both for real-space imaging [20] and imaging of the reciprocal space in a momentum microscope [21-23]. This achievement is the result of intense development at the Max-Planck Institute (MPI) for Microstructure Physics in Halle, Germany. The superior beam quality of the electron microscope is impressively evidenced by the $5 \times 10^{3}$ data points recorded simultaneously. Data points here refer to resolved $\left(k_{x}, k_{y}\right)$ pixels $\left(k_{x}, k_{y}\right.$ : transversal components of the momentum vector). This application is discussed in detail by Suga and Tusche [23].

The last case adds the third dimension to spin detection by implementing time-of-flight based parallel energy acquisition in a momentum microscope. This approach can be expected to yield the highest degree of parallelization in terms of the number of resolved $\left(k_{x}, k_{y}, E_{B}\right)$-voxels ( $E_{B}$ : binding energy). The development of this approach is not yet complete and some problems concerning chromatic aberration of the optics and the energy dependence of the spin asymmetry have yet to be solved. This promising approach is outlined in the outlook section and is discussed for the first time in the present article.

ToF spin detection without spatial resolution was pioneered in the 1990s by Müller et al. using a Mott detector [24]. Recently, this technique has been applied in the form of a very powerful set-up at the American Light Source (ALS), Berkeley [25]. An example demonstrating the performance of this achievement is given in Section 4. The time E spin section also covers the aspect of ultrafast spin detection. Photoelectron spectroscopy work on this issue is still rather sparse due to serious experimental obstacles, e.g. space-charge effects. As examples, we show data from a laboratory experiment with a fs-laser pump-probe with time resolution in the $30 \mathrm{fs}$ range [26] and a very recent benchmark experiment by Fognini et al. [27] at the free electron laser FLASH in Hamburg.

In Section 5 the $k$-space $\mathcal{E}$ time aspect will be discussed. We show results for a novel approach to mapping of the $E$-vs- $k$ spectral function, based on an imaging ToF momentum microscope developed at Univ. of Mainz in cooperation with MPI Halle using the optics described in $[22,23]$. Instead of the dispersive analyzer, a ToF section serves for energy discrimination and parallel energy acquisition. The optics are optimized for maximum resolution of the reciprocal image and facilitates a large zoom range of the analyzed sample region via selectable apertures (several $100 \mu \mathrm{m}$ down to the range of $1 \mu \mathrm{m}$ ) and of the momentum disc imaged simultaneously (diameter several $\AA^{-1}$ down to a fraction of a $\AA^{-1}$ ). Concerning the $3 D$ data acquisition, this instrument is related to ToF-PEEM [28] and the ToF-spectrometer family of instruments [29,30]. However, there are essential differences between the approaches, in particular with regards to the large energy range from the threshold to the HAXPES range (which will be addressed in the outlook section), the planar isochronous surfaces and the possibility to implement an imaging spin filter. In Section 5, we will also discuss a recent example of ultrafast probing of transient electronic states using a high-harmonic source at $h v=22 \mathrm{eV}$ [31]. In Section 6, the present status of these developments is extrapolated into the near future.

\section{Space and time: Imaging of fast processes using PEEM}

\subsection{Imaging magnetic polarization dynamics}

The understanding of the dynamic magnetization response caused by an external excitation is an important precondition for the design of magnetization reversal processes in magnetic storage and sensor devices. Magnetization patterns of interest usually involve domain structures and complex magnetic configurations [32]. The knowledge about the microscopic mechanisms determining the time-evolution of these non-collinear magnetization structures allows for example the improving of the writing speed of the cells in a magnetic random access memory (MRAM) device. To address this issue, the time-dependent behavior of magnetic domains and domain walls must be visualized with a combination of sufficiently high lateral and time resolution. This combination became available by methods based on polarized soft X-rays, namely, time-resolved X-ray photoemission electron microscopy (TR-XPEEM) [10-12,33-36] and time-resolved X-ray transmission microscopy (TR-XTM) [37,38]. The XPEEM permits a surface-sensitive approach to magneto-dynamic phenomena, whereas XTM yields bulk information. Both employ X-ray magnetic circular dichroism (XMCD) as contrast mechanism.

Characteristic sizes of the magnetic domains range up to the order of several micrometers. Therefore, a high time resolution accompanied by a moderate lateral resolution (several $100 \mathrm{~nm}$ ) has first been realized in optical techniques, such as magneto-optical microscopy (Kerr/Faraday microscopy) [39,40] and magneto-optical scanning near-field microscopy (SNOM) [41]. 
In a time-resolved PEEM, however, the lateral resolution may be better by almost an order of magnitude [42]. The time resolution is achieved by a stroboscopic technique where a pulsed illumination source is phase locked to an external magnetic field excitation. The stroboscopic technique limits the observable magnetization dynamics to reversible processes. Non-reversible dynamics can only be observed in steps with intermediate frozen quasi-equilibrium states. On the other hand reversible processes can be studied with a rather high time-resolution, which is in principle only limited by the pulse width of the probe pulses. Using all-optical pump and probe schemes time resolution below $100 \mathrm{fs}$ seems achievable by exploiting the magnetic dichroism in threshold photoemission $[43,44]$.

The highest time-resolution using external field excitation pulses has been achieved so far for single crystalline strip lines [45]. In this experiment the time-resolution was limited equally by the time duration of the probing X-ray pulses convoluted with electronic jitter, which is given e.g. by $20 \mathrm{ps}$ in the low-alpha mode at BESSY, and by the $40 \mathrm{GHz}$ band width of the excitation cabling and electrical feedthroughs corresponding to $25 \mathrm{ps}$.

Fast magnetic field pulses were applied via the Oerstedt field of a strip line passed by fast current pulses. The single crystalline Mo(110) strip lines were produced in the following way. In the first step, a $\mathrm{Mo}(110)$ epitaxial film $(200 \mathrm{~nm})$ was deposited on $\mathrm{Al}_{2} \mathrm{O}_{3}(11-20)$ in UHV by electron-beam evaporation. During Mo evaporation, the temperature of the substrate was kept constant at $1000 \mathrm{~K}$. In the second step, the strip line (coplanar waveguide with a width of $100 \mu \mathrm{m}$ of the central lead) and contacts were patterned by standard photolithography. The sample was again transferred into the UHV chamber and in the third step, the Mo strip line was cleaned by repeated cycles of heating in oxygen atmosphere up to $1300 \mathrm{~K}$, followed by flashing at $1800 \mathrm{~K}$ in UHV until the Auger electron spectroscopy signals of $\mathrm{C}$ and $\mathrm{O}$ originating from the Mo surface were below the detection limit. On this sample a Co layer (20 ML) and a Au capping layer (5 ML) were deposited by molecular beam epitaxy. Epitaxial growth of the Co layer was controlled by low energy electron diffraction (LEED). The Co/Au layer was kept thin enough in order to avoid electrical shorting of the strip line. The final structuring of the Au-passivated platelets on the central lead was performed by focused ion beam etching (FIB). The central lead was etched on a length of $1 \mathrm{~mm}$ to a width of $20 \mu \mathrm{m}$ in order to increase the Oerstedt field. The pulse generator delivered pulse amplitudes of $\sim 7 \mathrm{~V}$ with a rise time of less than $50 \mathrm{ps}$ (Fig. 1 top) at a frequency of $0.5 \mathrm{GHz}$ phase-locked with the synchrotron frequency. An adjustable electronic delay served to set the time of image acquisition with respect to the magnetic field pulse. Further details of the stroboscopic imaging approach performed in the photoemission electron microscope are described in [12,34]. The experiment was carried out using the UE52-SGM beam line at the BESSY II synchrotron radiation source (Berlin).

Due to the small damping in the $\mathrm{Al}_{2} \mathrm{O}_{3}$ substrate the field pulse shows an equally sharp leading and trailing edge, reproducing exactly the output of the pulse generator. The maximum field amplitude amounts to $6 \mathrm{mT}$. Co films grown on $\mathrm{Mo}(110)$ show a uniaxial anisotropy with the easy axis along the Mo [1-10] direction, that in turn is aligned with the strip line axis [46]. The anisotropy constant determined from Kerr magnetometry measured before the final FIB structuring is $K_{p}=0.47 \times 10^{5} \mathrm{~J} / \mathrm{m}^{3}$ corresponding to a saturation field of $\mu_{0} \mathrm{H}=50 \mathrm{mT}$. The deviation from the results measured for Mo capped $\mathrm{Co} / \mathrm{Mo}\left(\begin{array}{ll}1 & 10\end{array}\right)$ films might be attributed to the surface anisotropy induced by the Au capping. The uniaxial anisotropy defines the magnetization direction at equilibrium along the strip line. A homogeneous magnetization state as shown in Fig. 1(a) is typical for an epitaxial film. The dark color of the platelets indicates a magnetization component antiparallel to the incident X-ray beam before the field pulse
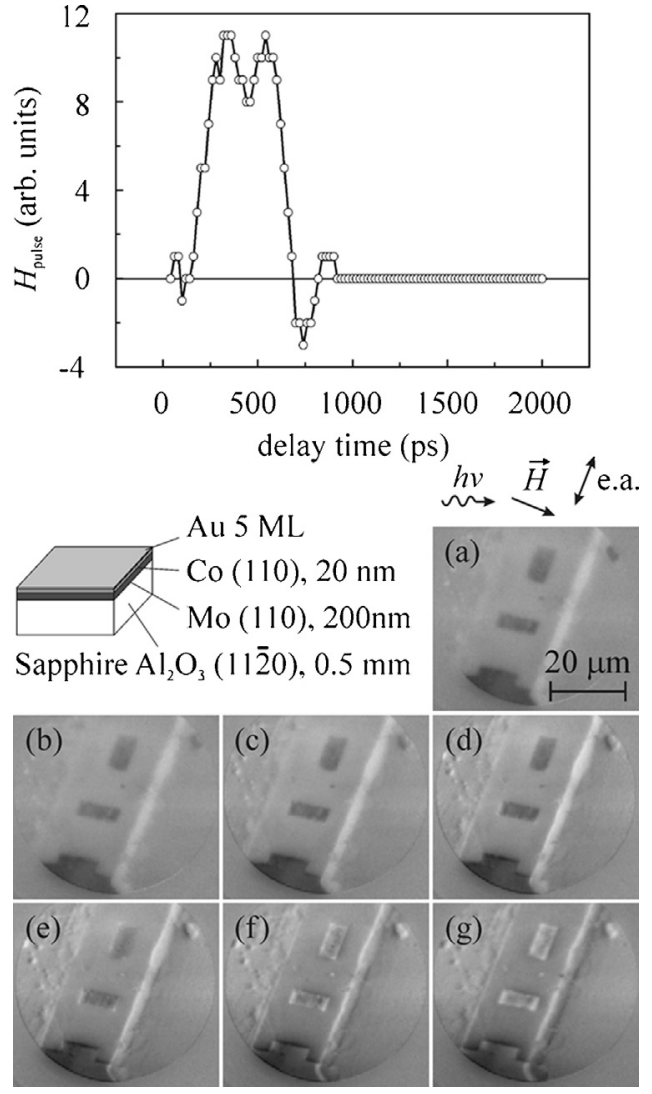

Fig. 1. Time-resolved XMCD-PEEM of epitaxial Co platelets on a single-crystalline $\mathrm{Mo}(110)$ strip line on $\mathrm{Al}_{2} \mathrm{O}_{3}(11-20)$. Top: Temporal profile of the magnetic field pulse $H_{\text {pulse }}(t)$. Bottom: Sequence (a-g) showing the change of magnetization state with time steps of $\Delta t=20 \mathrm{ps}$. The series was taken in the rising edge of the field pulse. Sizes of Co particles on the strip line are $5 \mu \mathrm{m} \times 10 \mu \mathrm{m}$ and $10 \mu \mathrm{m} \times 5 \mu \mathrm{m}$ (from [45]).

arrives. During the start of the field pulse we observe a reversal of the XMCD asymmetry revealing a change of sign of the horizontal magnetization component. Consequently, the magnetization has rotated by at least $45^{\circ}$ in Fig. 1 (g). This unexpected large response might be attributed to a decreased magnetic anisotropy due to the FIB etch process. A larger positive asymmetry (positive horizontal magnetization component) at the edges of the rectangle that are oriented perpendicular to the field direction indicate a stronger dynamic response of the magnetization at the edges where the field enters and leaves the magnetic particle. This is not surprising because the demagnetizing field destabilizes the magnetization component perpendicular to the free edges. Comparing Fig. 1(e) and (f) significant changes of the magnetization structure within $20 \mathrm{ps}$ are resolved, demonstrating the high time-resolution of the experiment.

The second example demonstrates how the combination of time and space resolution can be exploited to understand complex dynamic phenomena in magnetic nanostructures. In magnetic nanostructures with sizes larger than the magnetic exchange length the ferromagnetic order decomposes in domains [47]. The magnetization can of course be saturated parallel to a static external magnetic field. Alternatively, an oscillating microwave field can magnetize the particle perpendicular to the direction of the magnetic field [34]. This is a striking phenomenon because the microwave excitation increases the order in the particle against the common trend of increasing disorder and entropy. In this case the decrease of entropy is attributed to the constant throughput of energy. The external oscillating field is the energy source while the internal damping plays the role of the sink. 


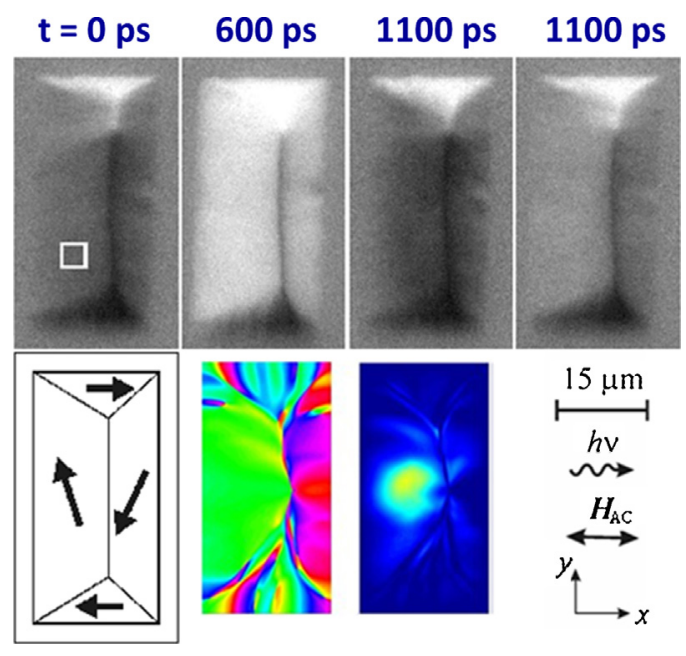

Fig. 2. Top: XMCD images showing the time evolution of the $x$-component of the magnetization (bright areas are magnetized to the right, dark areas to the left, white square indicates largest domain) in a Permalloy platelet ( $16 \mu \mathrm{m}$ width, $32 \mu \mathrm{m}$ length, and $10 \mathrm{~nm}$ thickness, placed on a coplanar waveguide). Bottom (from left to right): Sketches of the corresponding domain pattern, micromagnetic simulation with color-coded magnetization direction, Fourier map indicating the amplitude of the $1 \mathrm{GHz}$ oscillation mode and sketch of the geometry (adapted from [34]).

TR-XPEEM serves to understand this process in detail. In the rectangular platelet shown in Fig. 2, the equilibrium magnetization state is a symmetric flux-closure domain pattern, comprising two equally sized domains separated by a $180^{\circ}$ domain wall. The external field is applied along the short side of the platelet, thus exciting a precessional motion of the magnetization with a frequency of $1 \mathrm{GHz}$ slightly off-resonance. The system reacts by increasing one magnetic domain at the expense of the others, corresponding to a movement of the domain wall to the right. The final state is the uniform magnetization.

Snapshots of the time evolution of the magnetization $\boldsymbol{M}$ in the Permalloy platelet are shown in Fig. 2 ( $\boldsymbol{M}$ being denoted as arrows in the bottom left panel). The domains oriented parallel and antiparallel to the X-ray polarization vector (helicity vector being parallel to $\mathrm{h} v$ ) appear black and white, while the two domains oriented perpendicular to the helicity vector both appear gray. A $180^{\circ}$-Neél wall along the $y$-axis separates the two large domains with $\boldsymbol{M}$ upward (left) and downward (right). In the Néel wall, $\boldsymbol{M}$ is oriented to the left; thus, the Néel wall appears black. The intensity in the two large domains varies periodically, indicating the predominant excitation mode of a precession of the magnetization around the effective field axis directed parallel to the long side of the platelet. The system resembles a driven oscillator, and we observe the dynamical answer of the system to the periodic excitation. The basic mechanism, revealed by both stroboscopic imaging and computer simulation, is the decrease of the resonance frequency in the larger domain. This leads to larger energy dissipation in the magnetic particle, allowing increasing order with decreasing local entropy. The magnetization precession in the large domain mainly controls the energy dissipation in the particle. The system is excited with a significant oscillating field component of $1 \mathrm{GHz}$, i.e., just below the resonance frequency of the free running system of about $1.25 \mathrm{GHz}$. If the domain wall shifts to the right, the effective field determining the precession frequency and, consequently, the resonance frequency will decrease in the left domain and vice versa in the right domain. Therefore, the amplitude of the precession will increase in the left domain and decrease in the right domain as shown in Fig. 3. Since the precession energy is proportional to the square of the amplitude, the total energy has increased. Moreover, the domain with the larger amplitude has grown, which also helps

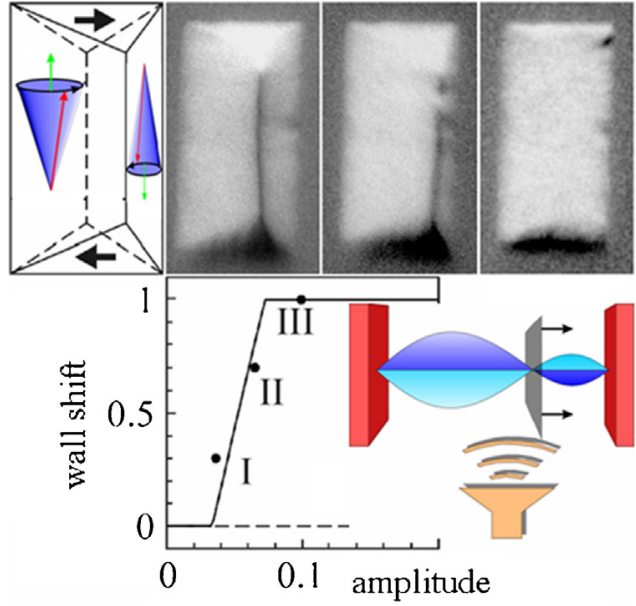

Fig. 3. Top: Sketch and snapshots of magnetic domain patterns at the time $t=0$ excited with increasing microwave amplitudes I, II and III as indicated in the bottom panel. The bottom panel shows comparison of an analytical model and experimentally observed mean shift of the central domain wall. The inset shows an analog mechanical model (adapted from [34]).

to increase the dissipated energy. The stray field energy resulting from the increasing magnetization finally balances the domain wall shift. The process needs an initial small symmetry breaking mechanism, which is attributed to imperfections of the platelet's shape or to vortex motion of the Bloch line as described in [10].

The reversal of the horizontal magnetization component at the upper end of the Néel wall in Fig. 2 indicates a magnetic vortex and is called Bloch line. This highly magnetically charged area emits spin waves upon microwave excitation. The spin waves are magnetostatic backward volume modes propagating parallel to the mean magnetization direction. The spin waves are visualized by plotting line profiles of the $x$-axis magnetization component vs time as grey scale maps (see Fig. 4). The line profiles along the $y$-axis were acquired to the left and to the right of the Bloch line (Fig. 4(a)). Connecting the points of constant phase, e.g. the maxima, directly visualizes the motion of the phase front. The slope represents the phase velocity of the spin wave. While the maxima near $0.7 \mathrm{~ns}$ represent a homogeneous excitation, the maxima near $1.5 \mathrm{~ns}$ indicate a propagating spin wave. On the left side the wave above the Bloch line (positive $y$ value) shows a positive velocity, while the wave on the right side below the Bloch line propagates into the opposite direction. In both cases the spin wave propagates along the mean magnetization direction. The velocity amounts to $15 \mathrm{~km} / \mathrm{s}$, which is large compared to reported values for permalloy structures and on the same order of magnitude as the spin wave velocity observed for CoFeB thin films [48].

\subsection{Imaging electric polarization dynamics}

Photoemission electron microscopy (PEEM) is one of the few methods offering experimental access to the electrical near-field upon significantly enhancing the photoemission yield $[13,14,49]$. Mapping of near-field distributions pushes the achievable space resolution for optical spectroscopy far beyond the diffraction limit. Details of the non-linear electron emission process have been discussed in [50,51]. The dependence on polarization strongly depends on the shape of the nanostructures and can thus be manipulated to obtain special functionalities [52]. The subwavelength resolution can be used in the case of spectroscopy in order to gain insight in spatially extended excitations and in the case of imaging thus enabling a visualization of propagating surface plasmons [15-17]. In the following we discuss prominent examples for both cases. 

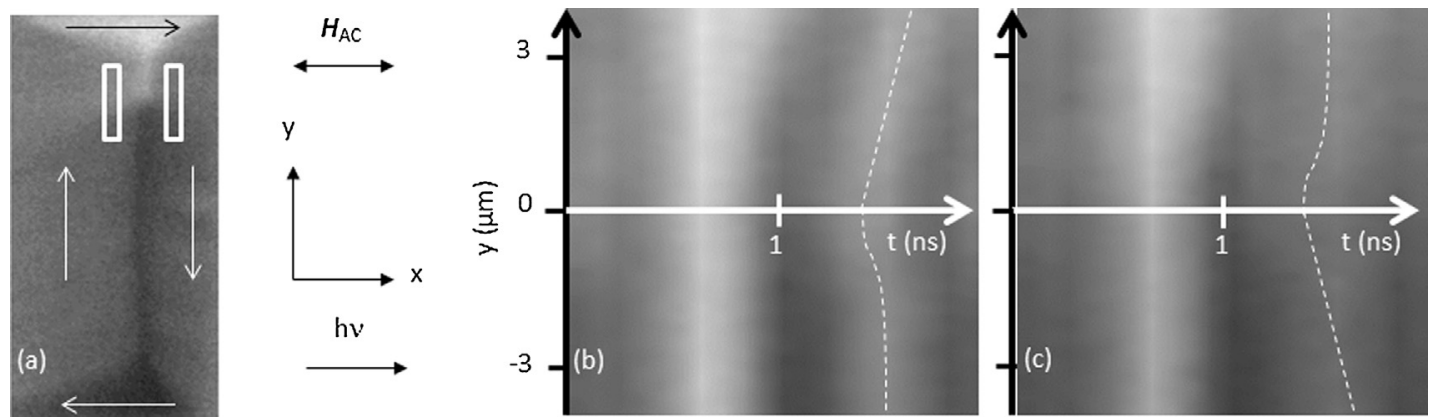

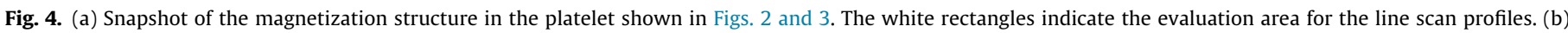

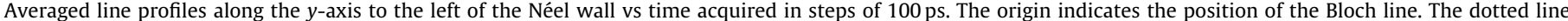

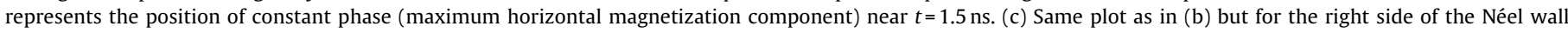
(adapted from [34]).

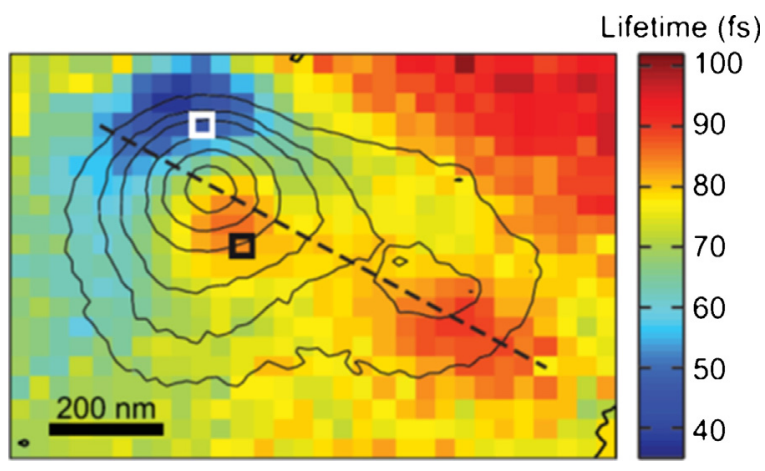

Fig. 5. The coherence lifetime is shown as a function of position. The map indicates the coherence lifetimes (false colors) for a hot spot (photoemission yield in black contour lines). The white and black squares mark locations of particularly long and short coherence times, and the dashed line indicates a cut through the two centers of a coupled localized plasmon (adapted from [56]).

Recently, subdiffraction imaging resolution obtained by reversible fluorophore saturation has attracted scientific attention [53]. This method allows for imaging but makes ultrafast spectroscopy challenging because the transitions need to be saturated. Near-field methods provide another way to achieve subdiffraction resolution [54], and enable time-resolved ultrafast experiments [55].

The first near-field optical spectroscopy using PEEM was introduced in [56]. While in a conventional four-wave mixing experiment, three ingoing optical waves are converted to one outgoing optical wave, the PEEM experiment exploits four-wave mixing employing four ingoing waves and detection by the nonlinear photoemission. The four waves in this experiment consisted of individually pulse shaped waves from a Ti-sapphire femtosecond laser with $1.4 \mathrm{eV}$ photon energy. Local spectra were recorded from a corrugated silver surface where the main advantage of PEEM is the parallel data acquisition allowing for a comparison of different locations within a single experiment. Fig. 5 shows the coherence lifetimes as a function of position for an intensity hot spot on the Ag surface. The main surprise in the experiment is that plasmonic phase coherence of localized excitations persisted for about $100 \mathrm{fs}$ and exhibited coherent beats. The observations are best explained by a model in which coupled oscillators lead to Fano-like resonances in the hybridized dark- and bright-mode response. The bright mode of the plasmonic excitation exhibiting a large dipole moment interacts strongly with the transverse radiation field and enables the effective manipulation of the system. The dark mode interacts not directly with the transverse fields and thus exhibits long lifetimes. This is the reason why nanoscale coherence trapping could be observed.
The fixed glancing angle of incidence of the light in a common PEEM setup makes the interpretation of the time-resolved investigation of propagation of surface plasmon polaritons (SPP) difficult. One problem is caused by the fact that by turning the polarization of the light, not only the in-plane component of the electric field is rotated. Due to the oblique incidence of the laser pulse the rotation of the light polarization also results in an additional field component perpendicular to the surface which influences the measurement by exciting plasmonic modes perpendicular to the surface of the nanostructures. In addition, the difference in the dispersion of the incoming light field and the SPP causes an interference pattern induced by a beating effect. A normal-incident PEEM setup [57] overcomes both problems, since the phase of the probe pulse would have the same value on every point of the investigated structure. A similar setup has been used to observe circular modes in nano-antennas [58].

Such a time-resolved photoemission microscopy experiment, referred to as femtosecond normal-incidence photoemission microscopy has been pioneered by Kahl et al. [57]. It was shown that the change from the commonly used grazing incidence to normal-incidence illumination geometry has a major impact on the achievable contrast and, hence, on the imaging potential of transient local near fields. As an example SPPs excited at the edge of Ag islands are imaged in normal and grazing light incidence geometry. The observed fringe spacing directly resembles the wavelength of the plasmon wave in the case of normal-incidence and thus provides a direct visualization of SPP wave packets propagating across a metal surface.

Fig. 6 shows examples for time-integrated imaging of 2PPE SPPs excited on Ag islands with excitation under different incident angles using a linear PEEM set-up with a small mirror in the back focal plane [59] and a low energy electron microscope used as a PEEM [60]. In Fig. 6(a) and (c), the micrometer fringe spacing (beating pattern) observed under grazing incidence is clearly visible. Under normal incidence, the contrast in Fig. 6(b) and (d) is entirely different. For grazing incidence, the observed periodic brightness modulation exhibits a pattern with a periodicity that depends not only on the used laser wavelength but also on the direction of the laser pulses and the propagation direction of the SPP. The contrast can be described by a Moiré pattern. The Moiré condition yields a fringe spacing depending on the angle between the propagation direction of the SPP and the incident light. This becomes visible e.g. in Fig. 6(a), where a narrow spacing of Moiré fringes occurs at the top and bottom of the $\mathrm{Ag}$ island while a wide spacing starts near the left edge. For normal incidence (Fig. 6(b)-(d)) the fringe spacing becomes identical to the SPP wavelength. Moreover, the propagation direction of the observed pattern is identical to the propagation direction of the SPP. Thus for most investigations of SPP wave packets the normal incidence illumination is advantageous, 

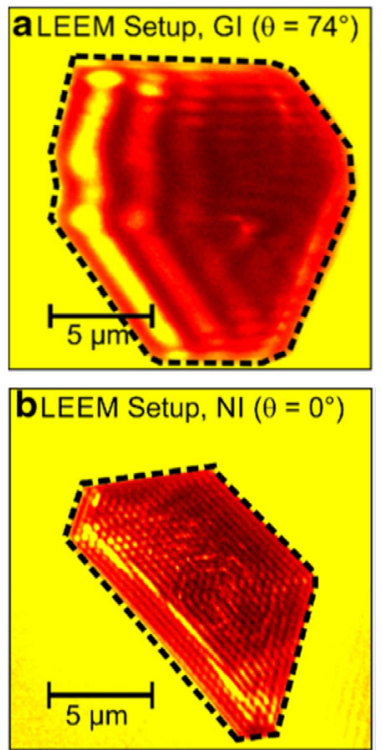
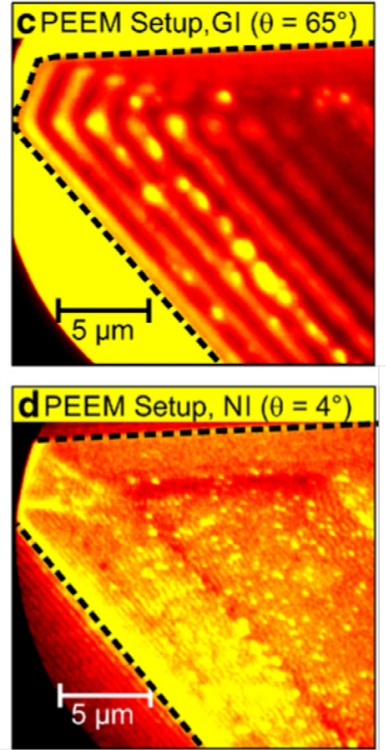

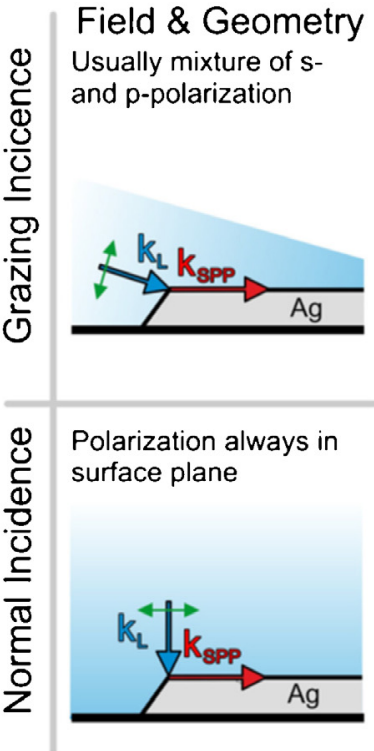

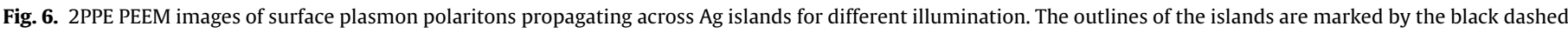

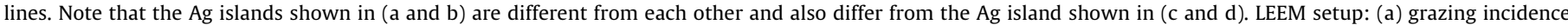

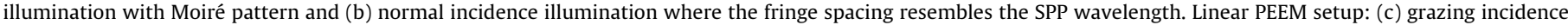

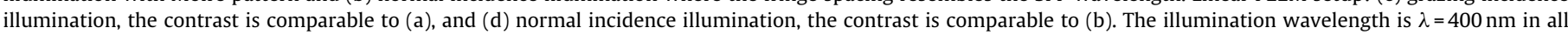

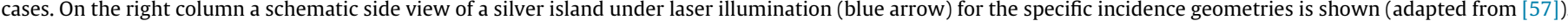

since it provides an easily interpreted high-resolution "snapshot" of the SPP wave. In particular, for time- and phase-resolved measurements, normal incidence illumination provides a direct access to propagating SPP wave packets by means of a cross-correlation signal.

\section{Space and spin: Multichannel spin detection}

Although state-of-the-art spin detectors have reached good overall performance and ability for vectorial analysis $[61,62]$, the basic concept of present spin polarimeters is not compatible with parallel-detecting electron spectrometers. The multichannel concept is based on the idea of preserving a two-dimensional electron distribution in the spin-polarized low-energy electron diffraction process. Recent developments towards multichannel spin detection in combination with a hemispherical analyzer [18] and with an emission electron microscope [20-23] exploit $k_{\|}$-conservation in low-energy electron diffraction, similar to an optical mirror, as illustrated in Fig. 7 for real and reciprocal space. The Ewald spheres and reciprocal lattice points correspond to specular reflection from an $\operatorname{Ir}\left(\begin{array}{lll}0 & 0 & 1\end{array}\right)$-surface at an incidence angle of $45^{\circ}$ and scattering energies of $39 \mathrm{eV}$ and $10 \mathrm{eV}$. With $\operatorname{Ir}(001)$ [63] and $\operatorname{Ir}(001)-\mathrm{Au}(1 \times 1)$ [64] spin-filter surfaces with high figure of merit and long lifetime in UHV have been found. For high-Z materials the diffraction process is highly spin selective, usable maxima of the spin asymmetry function reach $80 \%$ [64].

Implementation of such a spinfilter into the exit of a hemispherical analyzer constitutes multichannel spin detection. The basic principle is sketched in Fig. 8, schematically simplified by omitting all electron lenses. In brief, electrons from a large part of the exit field of the hemispherical analyzer are diffracted as a parallel beam from the spinfilter surface, retaining their lateral position information. The spin-filtered image is then recorded by a $2 D$ image detector, here either a delay-line detector (DLD) for
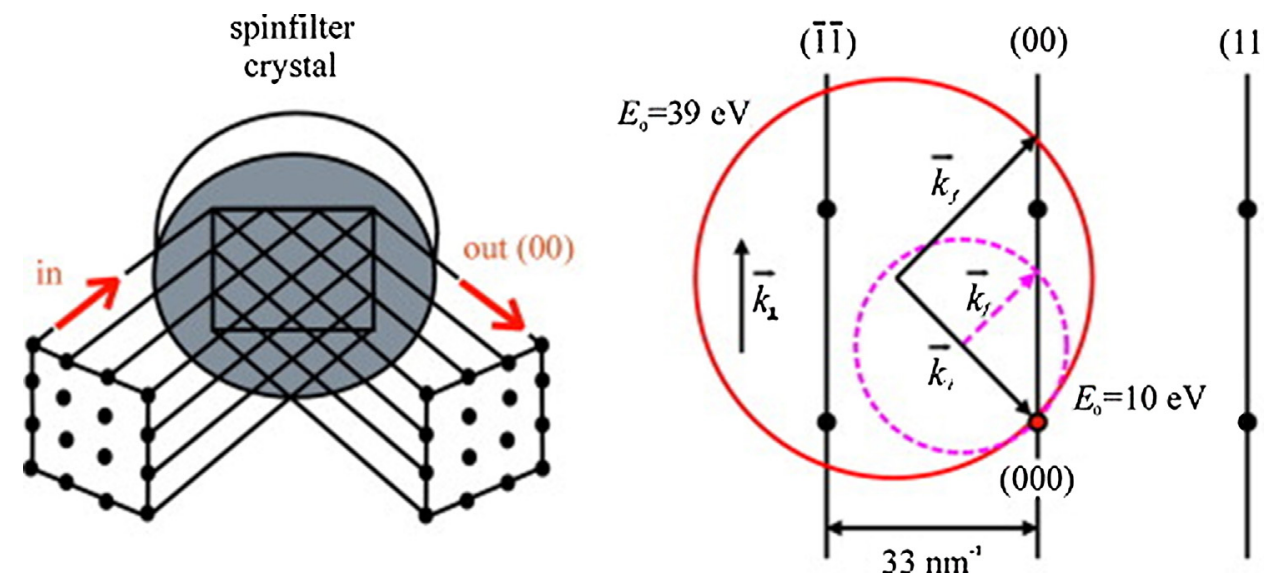

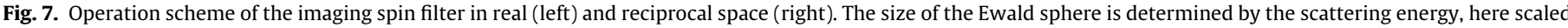

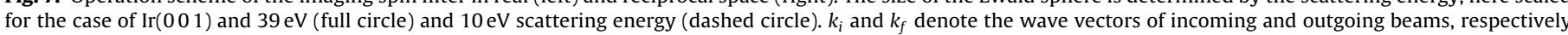
(from [63]). 


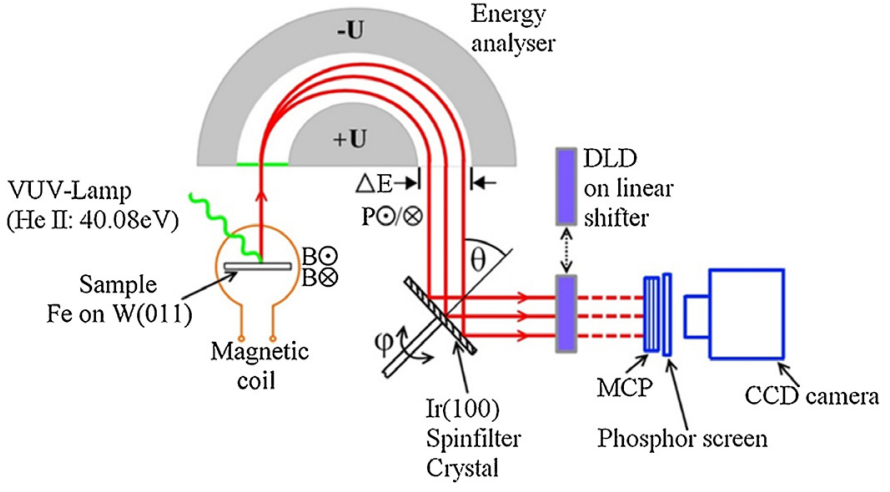

Fig. 8. Schematic section of a multichannel spin filter in the exit of a hemispherical analyzer. For sake of simplicity, all electron lenses are omitted. The $2 D$ electron distribution in the exit field is imaged via specular reflection at the spinfilter surface to the image detector, either a delay-line detector (DLD) or a multichannel plate (MCP) - phosphor screen - CCD camera combination (from [63]).

single-electron counting or an MCP-screen-CCD camera unit for optical detection. This setup is used for the study of thin ferromagnetic films $[18,63,65,66]$, where the scattering asymmetry can be easily reversed by switching the magnetization direction. A first commercial prototype is equipped with a spin-rotator lens [67] in order to reverse the spin asymmetry in cases like Rashba systems, where the initial polarization cannot be switched. A further possibility is to use a ferromagnetic spin filter [68], based on exchange scattering. A very successful strategy for asymmetry reversal is used in the spin-filtered momentum microscope developed at the MPI Halle. It exploits the fact that in several cases maxima with different signs of asymmetry occur within small intervals of the scattering energy. Changing between the two energies then leads to a "switching" of the asymmetry function. E.g. for the $\operatorname{Ir}(001)$ $\mathrm{Au}(1 \times 1)$ spinfilter a reversal from $-65 \%$ to $+57 \%$ is facilitated by changing scattering energy from 10.25 to $11.5 \mathrm{eV}$ [21].

A hemispherical analyzer can image a certain kinetic energy $(E)$ interval (typically $10 \%$ of the pass energy) and polar emission angle $(\theta)$ range (up to $\pm 15^{\circ}$ ) to the exit field. In total, $\sim 10^{4}(E, \theta)$ data points can be recorded in parallel [2,3]. The large field of view, the chromatic aberration and the strong astigmatism of the beam at the exit of the hemispherical analyzer pose a large challenge to the formation of a parallel beam on the spin filter crystal. In the outlook section we will discuss the obstacles and possible solutions in more detail. The electron-optical constraints caused a reduction of the number of simultaneously resolved $(E, \theta)$ data points to about $N=10^{3}$ in the first prototype [18].

Given this number of data points, the total performance depends on the spin asymmetry and reflectivity of the spinfilter crystal, being a function of scattering energy and scattering angle. The relevant quantity for the statistical performance of a polarimeter is its figure of merit [69]

FoM $_{\text {single }}=\frac{S^{2} I}{I_{0}}$,

with $S$ being the asymmetry function (Sherman function) and $I / I_{0}$ the reflectivity (ratio of scattered vs incoming intensity). The index "single" denotes that this definition holds for a single analyzed beam. In case of $\mathrm{N}$ resolved beams (each data point corresponds to a thin pencil beam), the total figure of merit (denoted by the index $2 D$ ) is obtained by numbering up:

$\mathrm{FoM}_{2 \mathrm{D}}=N\left\langle\mathrm{FoM}_{\text {single }}\right\rangle$,

where $\left\langle\mathrm{FoM}_{\text {single }}\right\rangle$ denotes the single-channel FoM, averaged over the used energy and angular range.
In a multichannel spin polarimeter the polarization is determined pixel-by-pixel from the difference in scattering intensities when reversing the asymmetry. For a geometry like in Fig. 7 there are various ways to reverse the asymmetry: by reversing the sample magnetization (if ferromagnetic samples are studied) or the photon helicity (in optical spin orientation), by rotation of the spinfilter-detector unit by $180^{\circ}$ about the incoming beam, by azimuthal rotation $\varphi$ (Fig. 8), by a magnetic lens acting as spin rotator, by switching the magnetization of a ferromagnetic spin filter, or by switching between two scattering energies with opposite sign of asymmetry (cf. Section 6.2). For each data point (pixel i,j) the spin polarization is determined as:

$P_{i j}=A_{i j} \frac{1}{S_{i j}}=\frac{I_{i j}^{+}-I_{i j}^{-}}{I_{i j}^{+}+I_{i j}^{-}} \frac{1}{S_{i j}}$,

where $S_{i j}$ is the Sherman function (apparatus function) and $A_{i j}$ the measured asymmetry derived from $I_{i j}{ }^{+}$and $I_{i j}{ }^{-}$, which denote the intensities in pixel $(i, j)$ measured before and after reversal of the asymmetry. Note that Eq. (3) is a generalization of the singlechannel definition [69]. When the technique of energy change between two working points is used for asymmetry reversal, the equation for the polarization is more complicated because the reflectivity changes when changing the working point. For this case we refer to [70], where this case is treated in detail. For the normal-incidence geometry of a multichannel Mott detector [71] it is possible to simultaneously detect scattered electrons under different azimuthal angles, with strongly tilted image plane.

Besides the detector performance, its implementation into certain electron-optical systems may cause further constraints (cf. Section 6.2). As a general rule, the "optimum multichannel spin polarimeter" should have a high $\left\langle\mathrm{FoM}_{\text {single }}\right\rangle$ in a maximum energy and angular range. A large energy range is desirable, e.g. behind a hemispherical analyzer or in the ToF application (cf. Sections 4.2 and 6.2). A large angular range enhances the tolerance for angular divergence, i.e. a maximum phase space interval of the analyzed beam. A third criterion, important for practical work, is the lifetime of the spinfilter surface. An outstanding system in this respect is the Au-passivated $\operatorname{Ir}\left(\begin{array}{ll}0 & 01\end{array}\right)$-surface as studied by Kirschner et al. [64], characterized by a lifetime in UHV of several months. In the Halle-type of momentum microscope with dispersive energy filter this type of spin-filtering electron mirror presently yields $5 \times 10^{3}$ $\left(k_{x}, k_{y}\right)$ data points in parallel [21-23]. In this instrument, sequential acquisition of $k_{\|}$-distributions at many energies yields the complete spin-resolved valence band structure. Targeting long-term stability, a theoretical study of multichannel Mott scattering at $40 \mathrm{keV}$ has been performed recently [71]; it will be addressed in the outlook section.

On the quest to maximum performance, asymmetry and reflectivity maps have been measured for four systems in the relevant energy and angular range, clean [63] and Au-passivated $\operatorname{Ir}(001)$ [64] and $W(001)$ [72], exploiting spin-orbit asymmetry, and $\mathrm{Fe}(0001)-\mathrm{O}(1 \times 1)$ using exchange asymmetry [68]. The latter system is characterized by an extremely low scattering energy around $3 \mathrm{eV}$ and has not yet been used in a multichannel spin polarimeter. Here we report results for $\mathrm{W}(001)$ in an extended angular and energy range. Diffraction from this surface benefits from a larger coherence length of the reflected beam due to smaller mosaicity or mosaic spread of the $\mathrm{W}(001)$ surface in comparison to the $\operatorname{Ir}(001)$ surface. This is advantageous for resolution of a high number of data points.

Fig. 9 shows the landscape of asymmetry (Sherman map) and figure of merit in the plane of scattering angle vs scattering energy ( $(a, b)$ experimental, $(c, d)$ theoretical). In all previous studies employing the $W(001)$ imaging spin filter $[18,20,65,66,73]$ the scattering angle was fixed at $45^{\circ}$ (circle in $(a, b)$ ). At this angle the 

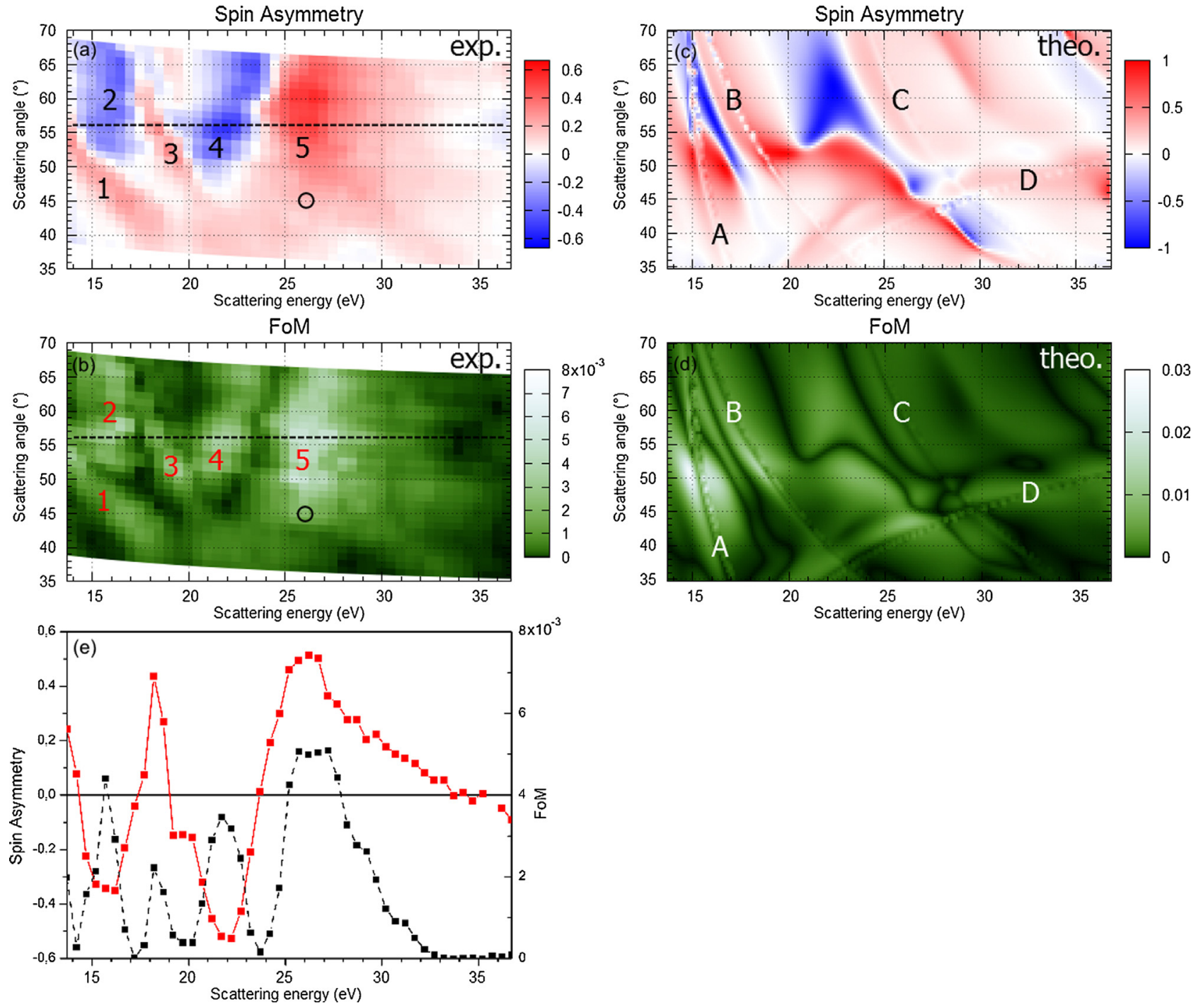

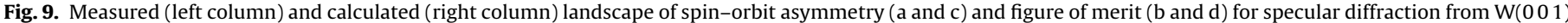

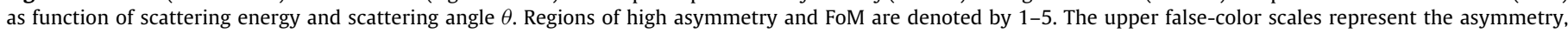

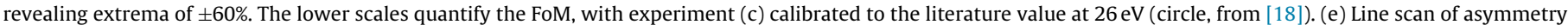
(full) and FoM (dashed) at $56^{\circ}$ scattering angle (after [72]). Figure courtesy D. Kutnyakhov.

asymmetry shows a maximum of $43 \%$ and a reflectivity of $1.2 \%$ (at $26 \mathrm{eV}$ ), resulting in $\mathrm{FoM}_{\text {single }}=2.2 \times 10^{-3}$. Close inspection of Fig. 9 reveals that asymmetry and FoM substantially increase towards higher scattering angles. The profile of the asymmetry function which is unipolar at $45^{\circ}$ becomes bipolar above $50^{\circ}$ as shown in line-scan (e). The positive maximum of region 5 is $+66 \%$ at $26.5 \mathrm{eV}$ and the negative extremum of region 4 is $-57 \%$ at $22 \mathrm{eV}$. At this scattering angle the asymmetry can be switched between $-53 \%$ and $+51 \%$ within an energy interval of $4 \mathrm{eV}$. Moreover, the regions of high asymmetry and FoM have a useful energy width of $3 \mathrm{eV}$.

Despite the fact that the $\mathrm{W}(001)$ crystal needs to be flashed about once every hour in good UHV, its spinfilter performance bears several advantages, e.g. easy surface preparation, no reconstruction of the clean surface and large asymmetry. The ab-initio calculation (by $\mathrm{H}$. Ebert et al.) shows good qualitative agreement with the experimental asymmetries and FoM patterns. The sharp lines labelled A, B and C in (c,d) denote surface-barrier induced resonances of the reflected intensity associated with the emergence threshold of non-specular LEED beams, D denotes a bulk-diffraction feature. In the experiment they are not resolved because of the limited transfer width of the experimental set-up which in turn is due to the electron optics, the electron source and possible misalignment of the $\mathrm{W}(100)$ surface. The dispersion of the resonances originates from multiple scattering, which becomes important at low kinetic energy of the reflected electrons. A kinematic model considering only single scattering events fails to describe the dispersion correctly. For a detailed discussion of surface-barrier resonances, we refer to [21].

The gain in data acquisition speed is crucial for spin-resolved spectroscopy of samples that are radiation-sensitive (minimum dose) or chemically reactive (minimum acquisition time). Recently, the multichannel spin detector made it possible to study Heusler compounds whose high surface reactivity is prohibitive for conventional single-channel spin detectors. An example from the group of M. Jourdan is shown in Fig. 10. $\mathrm{Co}_{2} \mathrm{MnSi}$ Heusler thin films were grown by means of molecular beam epitaxy on an $\mathrm{MgO}\left(\begin{array}{ll}1 & 0\end{array}\right)$ substrate; film quality was controlled by reflection high energy electron diffraction. Fresh samples were immediately transferred to the spectrometer chamber, equipped with a He discharge lamp $(h v=21.2 \mathrm{eV})$ and a hemispherical energy analyzer with multichannel spin filter (W(001) type with $45^{\circ}$ scattering angle). The measurement was performed at room temperature. A large spin 


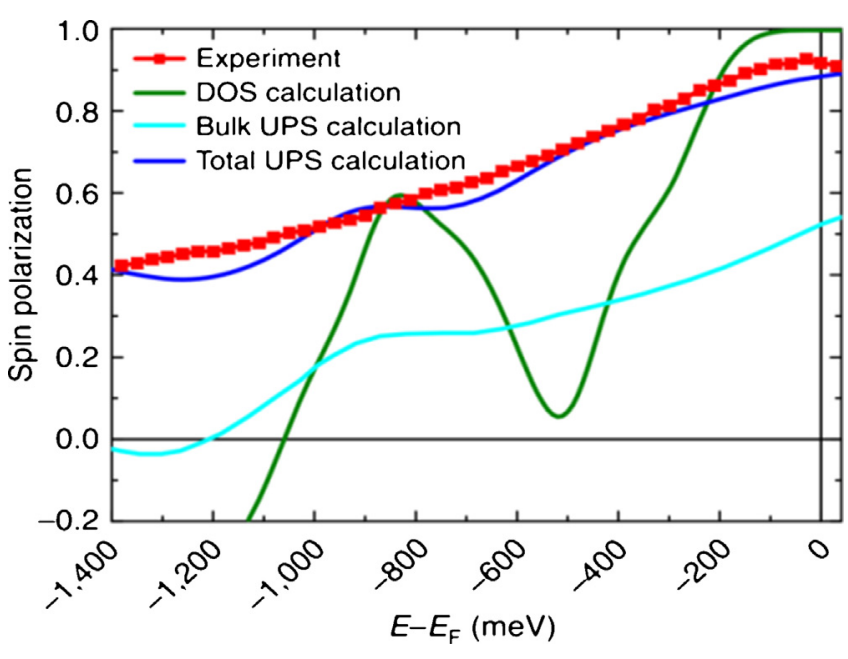

Fig. 10. Spin polarization of photoelectrons close to the Fermi edge of an epitaxial film of the Heusler compound $\mathrm{Co}_{2} \mathrm{MnSi}$ measured with the multichannel spin detector (squares). For comparison, theoretical calculations are included: purely DOS-derived spin polarization, UPS assuming bulk states and UPS including additional surface-state contributions (from [65]).

polarization of 93\% was measured at the Fermi energy, proving the half metallicity of this Heusler compound [65]. The related compound of $\mathrm{CO}_{2} \mathrm{MnGa}$ was studied in the same setup and a polarization of only $50 \%$ was found, excluding half-metallic behavior [73a]. Such experiments are important for materials design for spintronics devices and for sensitive tests of theoretical calculations. The theoretical curves were obtained using the SPR-KKR package with Perdew-Burke-Ernzerhof functional and dynamical mean field theory [73b]. Comparison with the calculated curves in Fig. 10 reveals that there is perfect agreement with experiment only if the surface contribution is properly taken into account in the photoemission code.

Heusler compounds are a perfect testing ground for the multichannel spin detector because the increased performance facilitates very fast measurements. Proving half metallicity with a simple laboratory photon source is hardly possible with a conventional single-channel spin detector due to the fast contamination of the reactive Heusler surface. The non-linearity of the polarization formula (Eq. (3)) leads to a rapid drop of the spin polarization due to contaminations. If only $10 \%$ electrons with the wrong spin orientation (due to contamination or diminishing of surface magnetism) would be admixed, the threshold value of $93 \%$ would already be reduced to $74 \%$. The hemispherical analyzer in this experiment (Leybold-type spectrometer) is not capable of high angular resolution, so the band dispersion was not accessible.

\section{Time and spin}

\subsection{Ultrafast spin processes}

Ultrafast demagnetization of a ferromagnet observed by heating the electron gas with femtosecond laser pulses [74] has initiated intense research activities. Experiments on the transition-metal ferromagnets cobalt, iron, and nickel have unambiguously established typical demagnetization times between 100 and $300 \mathrm{fs}$ after ultrafast optical excitation [74-77]. Therefore, the demagnetization time is much shorter than the typical relaxation between electron and lattice temperature. The Elliott-Yafet [78] spin scattering process involving the spin-orbit interaction has been considered the most important mechanism. More generally, electron-phonon scattering was widely considered to be essential for the demagnetization process. Contrarily, superdiffusive spin transport has

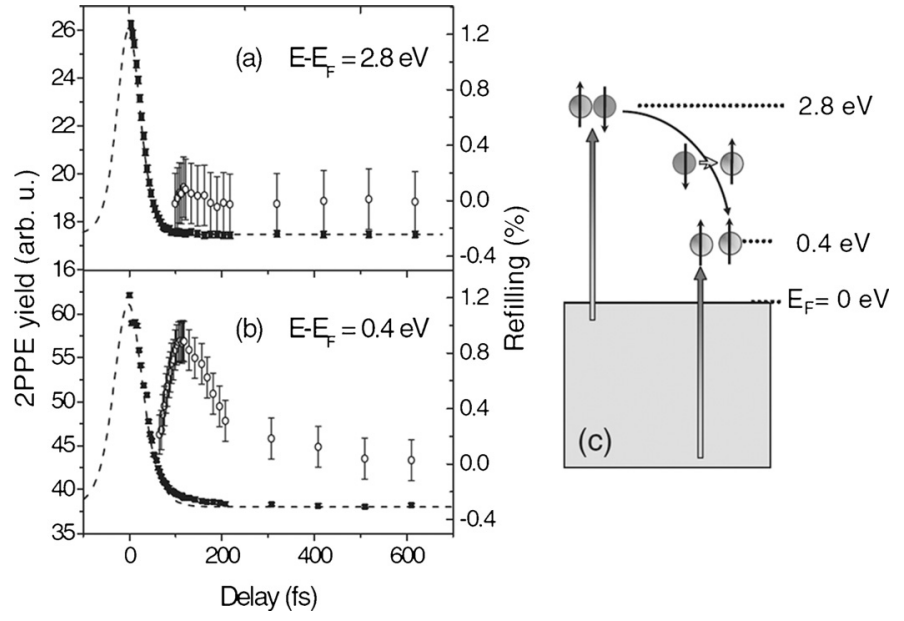

Fig. 11. $2 P P E$ yield at $h v=3.1 \mathrm{eV}$ from an intermediate state with an energy of (a) 2.8 and (b) $0.4 \mathrm{eV}$ as a function of the delay time between the pump and the probe pulse. The fit of the experimental curves with a rate-equation model is plotted with dashed lines. On the right scale the refilling percentage is shown. (c) Schematic picture of a possible spin-dependent refilling process of the intermediate state at $0.4 \mathrm{eV}$ including the Elliott-Yafet spin-flip process (from [26]).

been considered as an alternative mechanism [79-81]. While the explanation of ultrafast demagnetization is an ongoing research topic [82], time- and spin-resolved photoemission spectroscopy may provide an excellent experimental tool to clarify the different scattering mechanisms responsible for demagnetization.

Spin- and time-resolved photoemission measures the dynamics of single electron spin-flip processes, which are responsible for the observed ultrafast demagnetization times. Besides the ElliottYafet spin-flip scattering with impurities or phonons [78], the main mechanisms that flip the spin of an excited electron in a ferromagnet are Stoner excitation and inelastic scattering with magnon excitation. In particular excitation energies just above the Fermi level have to be considered. In this energy range spin-wave scattering clearly dominates Stoner excitation processes [83]. Using spin-resolved 2PPE spectroscopy the role of Elliott-Yafet scattering with respect to magnon scattering can be addressed.

In the experiment reported in [26] femtosecond pulses with photon energy of $3.1 \mathrm{eV}$ (fluence $3 \mu \mathrm{J} / \mathrm{cm}^{2}$ ) are used. The photon beam is divided into two equally intense, collinear and orthogonally polarized beams with an adjustable time delay. The s-polarized pump pulse excites electrons from the ground state into an unoccupied intermediate state. The delayed $p$-polarized probe pulse then excites the electrons above the vacuum level. The photoemitted electrons are analyzed in normal emission geometry using an electrostatic electron analyzer. The sample is a $10 \mathrm{~nm}$ thick epitaxial $\mathrm{Co} / \mathrm{Cu}(100)$ film that is remanently magnetized in two opposite magnetization directions. The spin-polarization is determined by a $\mathrm{W}(100)$-type spin detector. The recorded quantity is the number of excited electrons at a defined kinetic energy as a function of the time delay between the pump and the probe pulse. Fig. 11 shows the delay-time dependent intensity of two intermediate states with energies of $2.8 \mathrm{eV}$ and $0.4 \mathrm{eV}$ above $E_{F}$, respectively. In case the 2PPE signal is governed exclusively by an exponential population decay of the intermediate state, the experimental data can be fitted by a rate-equation model. Whereas the data at $2.8 \mathrm{eV}$ intermediate energy (Fig. 11(a)) are almost perfectly described by this model, the data at $0.4 \mathrm{eV}$ (Fig. 11(b)) reveal significant deviations. This deviation is explained by a refilling of the intermediate state by secondary electrons at temporal delays between 50 and 200 fs (see Fig. 12). The most relevant refilling mechanism is the scattering of an optically excited electron from a higher excited state with an occupied state below $E_{F}$. 


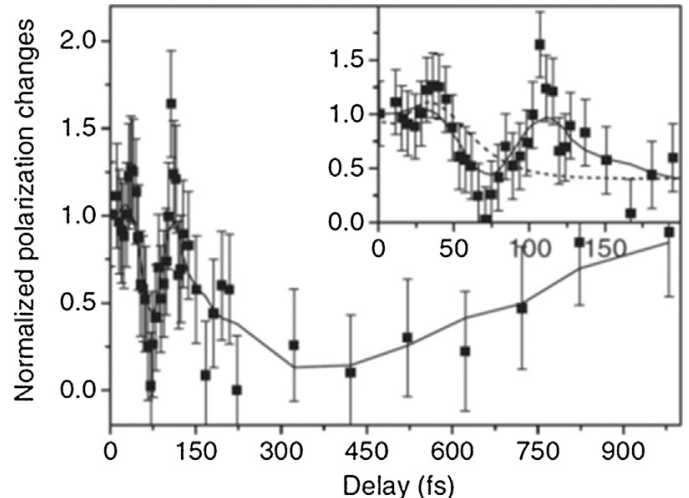

Fig. 12. Time dependence of the spin polarization of electrons in the intermediate state at $0.4 \mathrm{eV}$ above $E_{F}$. Inset: Zoomed picture of the same curve for time delays between 0 and $200 \mathrm{fs}$ and result of the numerical model discussed in the text (dashed line) (from [26]).

Fig. 12 shows the dependence of the in-plane polarization of electrons in the intermediate state at $0.4 \mathrm{eV}$ above $E_{F}$. It is expected that the dynamical single-particle occupation can be described again by a rate equation including cascade electronic scattering events. The result of the simulation for delays up to $200 \mathrm{fs}$ is shown in the inset (dashed line), together with the measured data. Clearly, good agreement between experiment and calculations is achieved only for the first $100 \mathrm{fs}$. The spin polarization first increases from its value at zero delay to a maximum close to $30 \mathrm{fs}$ which is due to the longer lifetime of majority electrons compared to the lifetime of minority electrons. This means that minority electrons depopulate the intermediate state at a faster rate than majority electrons, leading to a transient increase in the spin polarization (spin-filter effect). Between 30 and $75 \mathrm{fs}$, the spin-filter effect starts to become irrelevant, and the polarization decreases to the background value given by the spin polarization excited by the pump and probe pulses separately. The successive increase of the spin polarization in the experiment, with a maximum around $120 \mathrm{fs}$, is not reproduced by the simulation because the simulation does not consider spin-flip processes. The intermediate state at $0.4 \mathrm{eV}$ above $E_{F}$ is refilled by secondary electrons originating from energetically higher-lying minority electrons which have a higher probability than majority electrons to scatter inelastically with a magnon and thereby flip their spin, turning into majority electrons and, thus, causing an increase in the measured spin polarization (see Fig. 12). Therefore, it was concluded that even if microscopically spin-wave excitation plays an important role in the ultrafast spin dependent electron dynamics, the ultrafast demagnetization of the studied thin Co film is mainly due to Elliott-Yafet type spin-flip processes.

The direct observation of ultrafast spin-related changes of occupied valence states remains an experimental challenge. Ultraviolet or X-ray pulses cause electrons to be emitted directly in one step from the sample into vacuum. The spin polarization is detected by a spin polarimeter. Laser-based femtosecond light sources reach a photon energy of $6 \mathrm{eV}$ and the photoemission experiment can probe only the top $1 \mathrm{eV}$ of the valence band [84]. X-ray pulses with pulse lengths of $100 \mathrm{fs}$ from synchrotron [77] or high-harmonic [85,86] sources have a limited intensity inhibiting to-date a spin-resolved photoemission experiment. A novel approach is to use soft X-ray pulses from a free electron laser (FEL). First results in this direction using femtosecond pulses with a photon energy of $182 \mathrm{eV}$ are reported in [27]. The principle of such an experiment is shown in Fig. 13. An epitaxial Fe(1 10$) / \mathrm{W}(110)$ film serves as the magnetic sample. The Fe film has a magnetic in-plane easy axis along the [1-10] direction and yields a remanent single domain state. The
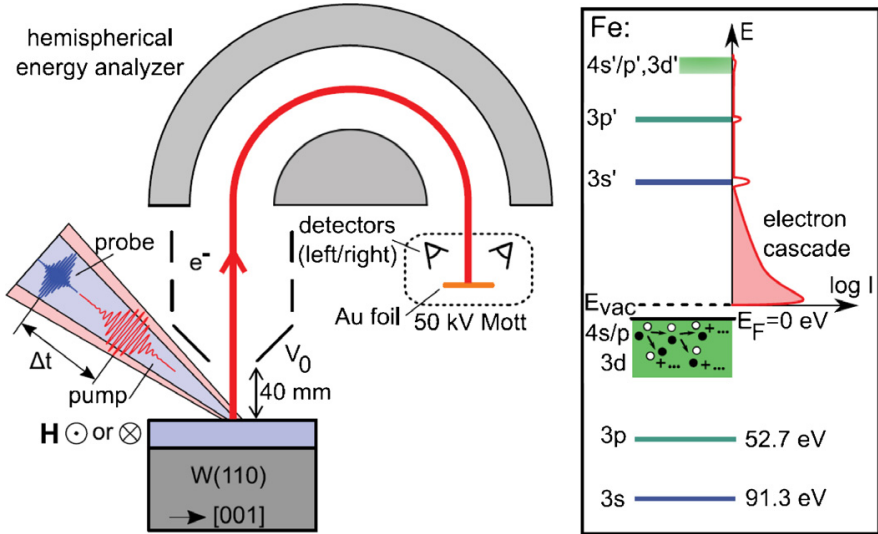

Fig. 13. Sketch of the spin-resolved experiment at the free electron laser FLASH, Hamburg. The iron film is excited by an $800 \mathrm{~nm}$ pump pulse. The subsequent FEL probe pulse generates an electron cascade which passes through a hemispherical energy analyzer. The spin polarization is measured with a Mott detector. An electric field of $2.5 \mathrm{kV} / \mathrm{m}$ is applied between the sample and the first lens element to accelerate the electrons towards the electron optics. The right panel shows the energy levels involved and the generation of the electron cascade with the electron yield denoted as I (from [27]).

change of the magnetization is measured by a pump-probe technique where the sample is demagnetized by an $800 \mathrm{~nm}$ laser pulse and probed by the $182 \mathrm{eV}$ FEL pulse. Photoelectrons are created by excitation with the FEL and their spin is measured by a Mott spin detector.

In the experiment of [27] the time-dependent spin polarization of secondary electrons is studied. The intensity of secondary electrons leaving the sample surface is an order of magnitude larger than electrons from the valence band and is therefore easier to detect. Secondary electrons originate from everywhere in the valence band and thus their spin polarization is an average measure of the actual spin polarization of the whole valence band. The spin filtering effect, i.e. minority electrons are scattered more effectively than majority electrons, further increases the detected spin polarization. The authors of [27] argue that the spin polarization of the secondary electrons represents the "total" magnetization of the sample, even in the pumped state.

Although the peak intensity of the FEL is orders of magnitude larger than any other source, the experiment is still time-consuming. In order to understand the limitations we discuss the parameters of the experiment in detail. The experiment has been performed at the PG2 beamline [87] of the free electron laser in Hamburg (FLASH). The ultrafast demagnetization is excited by a Ti-sapphire laser with $1.4 \mathrm{eV}$ photon energy and $120 \mathrm{fs}$ pulse width. Photoelectrons are emitted by $50 \mathrm{fs}$ probe pulses of an energy of $182 \mathrm{eV}$. The FEL pulse energy on the sample is fluctuating in the range of 6-300 nJ per pulse. Both lasers are $p$-polarized and impinge collinearly, under $45^{\circ}$ onto the sample. The spot-size of the pump beam $\left(0.07 \mathrm{~mm}^{2}\right)$ is larger than the FEL spot-size $\left(0.02 \mathrm{~mm}^{2}\right)$. The resulting incident pump energy density is $12 \mathrm{~mJ} / \mathrm{cm}^{2}$. The relative arrival time of pump and probe pulses is monitored with a streak camera and an electro optical beam arrival time monitor for the electrons in the FEL. This way the measurement can be corrected for drifts and the inherent time jitter of the FEL. The FEL is operated in burst mode where the machine delivers bursts of pulses at a rate of $10 \mathrm{~Hz}$. Each burst consists of 300 pulses separated by $1 \mu \mathrm{s}$. On every second FEL pulse, the film is excited by the pump laser. The un-pumped events provide a measurement of the full magnetization as a reference. For normalization, the sample's magnetic orientation is reversed between bursts by magnetic field pulses. The $3000 \mathrm{X}$-ray pulses in one second may excite just a few electrons per pulse in order to avoid the influence of space charge in the electron 

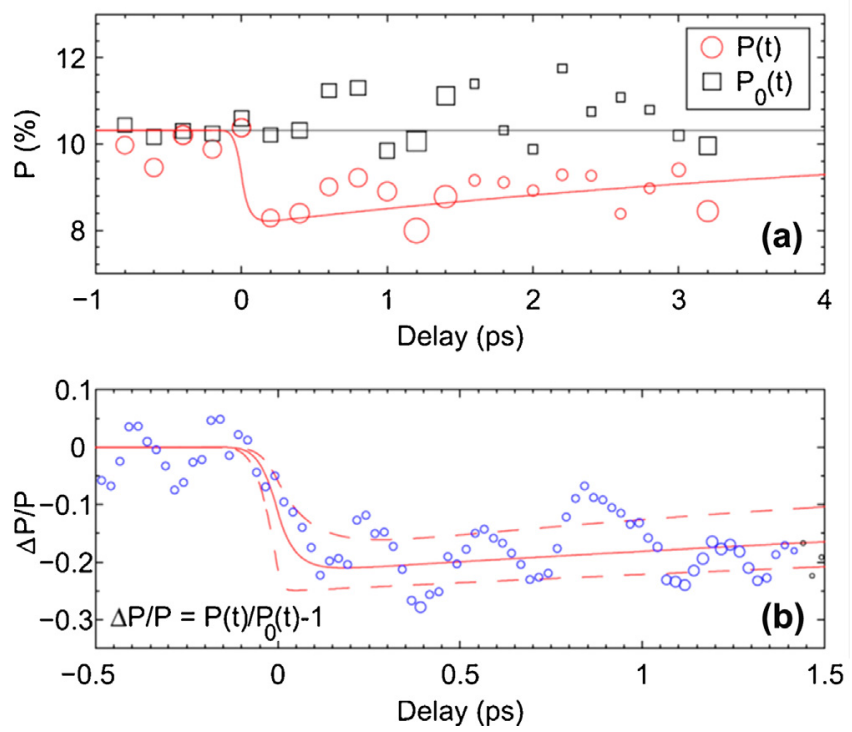

Fig. 14. (a) Polarization as a function of pump-probe delay for the pumped (red) and un-pumped (black) data points. The data have been binned to $200 \mathrm{fs}$. The average polarization is shown for the un-pumped sample (black line), and the fit from (b) is shown for the pumped sample (red line). The diameter of the data points represents their weight being proportional to the number of recorded FEL pulses. Notice that more FEL pulses have been recorded for the range of -0.5 to 1.5 ps resulting in less noise in this region. (b) The relative magnetization loss $\Delta P / P_{0}$ is smoothed. The apparent oscillations are caused by noise and not by the filtering process. The red curve is a fit to the measured values and the red dashed lines represent the standard deviation boundaries (from [27]).

analyzer optics [88]. The transmission of the electron analyzer was increased by a high pass energy of $100 \mathrm{eV}$ and a larger entrance slit thus measuring an energy band of $5 \mathrm{eV}$. Given the low figure of merit for the Mott detector, the experiment then still requires several hours of data acquisition.

Fig. 14(a) shows the polarization of the pumped (red) $P(t)$ and un-pumped sample $P_{0}(t)$ (black). The data have been binned to a temporal resolution of $200 \mathrm{fs}$. Panel (b) shows the laser induced magnetization loss $\Delta P / P_{0}$ as a function of the pump-probe delay. The result of the fit to an exponential demagnetization and recovery scenario leads to an exponential decay constant of $45 \mathrm{fs}$ and the minimum of the demagnetization lies at $180 \mathrm{fs}$. Hence, the experiment confirms the time scale of ultrafast demagnetization and rules out a transfer of spin to angular momentum.

As the incident intensity cannot be easily increased because of the space charge effect in combination with the limited pulse rate of the FEL, future developments must involve improvements at the detector. This may include both a higher single channel figure-of-merit of the spin detector beyond the performance of the classical Mott detector, the implementation of multichannel detection schemes as described in Section 3 and, finally, a multichannel detector as discussed in Section 6.3.

\subsection{Spin detectors with time-of-flight energy dispersion}

The technique of combining a spin detector with a time-offlight spectrometer was pioneered by N. Müller et al. exploiting the time structure of synchrotron radiation [24]. This setup used a Mott detector in combination with time-resolved detection behind a low-energy drift tube. Its energy resolution was about $300 \mathrm{meV}$. In fact this instrument was the first (1D-) multichannel spin detector with approximately 800 time points being resolved simultaneously, thereby compensating for the low inherent single-channel figure of merit $\left(\mathrm{FoM}_{\text {single }}=4 \times 10^{-5}\right)$ of the Mott detector operated at $45 \mathrm{keV}$ scattering energy. The development combined the know-how on spin detection from the Bielefeld group with the excellent ToF expertise of U. Becker et al. [89]. This instrument was very successful in the spin analysis of Auger electrons in gas-phase experiments. Applying the definition of the multichannel figure of merit from Section 3 we arrive at $\mathrm{FoM}_{1 \mathrm{D}}=N \times \mathrm{FoM}_{\text {single }}=0.03$.

The same idea was taken on more recently in a development at the ALS (Lawrence Berkeley National Lab, USA) by C. Jozwiak et al. [90], employing an exchange polarimeter for spin-resolved ARPES on solid samples. The analyzer combines the efficiency of low-energy exchange scattering spin detection (e.g. [91]) with parallel energy recording via ToF detection. Extremely powerful is the combination of this spin-ToF analyzer with a cavity-dumped Ti-sapphire laser [25]; a schematic drawing of this instrument is shown in Fig. 15. Spin polarimetry is based on low-energy exchange scattering from a ferromagnetic thin film of Co. Two ToF-branches allow for rapid change between spin-integral and spin-resolved measurements. The lens system additionally features a $90^{\circ}$ bandpass filter, thus removing unwanted parts of the photoelectron distribution. It allows the ToF technique to be performed at low electron drift energy and high energy resolution within a wide range of experimental parameters. Using this instrument surprising spin-polarization effects have been observed in the photoemission from topological insulators [92], providing insight into the physics of these fascinating materials.

Owing to its small Rashba spin splitting along with almost 100\% spin polarization, the Shockley surface state of $\mathrm{Au}(111)$ is a very good test case providing information about energy and momentum resolution of a spin-resolving analyzer. Fig. 16 shows a measurement taken with the laser setup of Fig. 15. The spin up and spin down partial spectra are marked in blue and red colors, respectively; the total intensity is the black curve. The measurement was done at $20 \mathrm{~K}$, acquisition time was only $3 \mathrm{~min}$ for this spectrum. A fit of the Fermi edge revealed an instrumental energy resolution of $\sim 14 \mathrm{meV}$. The angular resolution is $\pm 1^{\circ}$ which translates into a total momentum resolution of $0.02 \AA^{-1}$. The measured photocurrent was $\sim 150 \mathrm{pA}$, which proves the high quality of the fourth harmonic conversion. Using the parameters stated in [25] one can estimate for this instrument $\mathrm{FoM}_{1 \mathrm{D}}=N \times \mathrm{FoM}_{\text {single }}=0.125$.

\section{5. $k$-Space and time}

\subsection{Time-of-flight momentum microscopy}

In this section we address a novel way of $k$-resolved photoelectron spectroscopy aiming at a maximum degree of parallelization in data acquisition. Time-of-flight momentum microscopy was developed at the University of Mainz in cooperation with the MPI for Microstructure Physics in Halle. This concept will be most important for time-resolved studies of transient states and for spinresolved spectroscopy. The underlying idea makes use of a basic concept of microscopy: in imaging optical systems the reciprocal image represents the distribution of the transversal momentum components (in mathematical language it is also termed Fourier image). Owing to $k_{\|}$-conservation in the photoemission process, the reciprocal image formed in the backfocal plane of a cathode lens directly shows the surface-projected band structure inside the crystal. The basics of the electron optics of cathode-lens type microscopes are discussed in detail by E. Bauer (e.g. in [93]). Detection of the reciprocal image with maximum resolution is the essence of momentum microscopy as pioneered in J. Kirschner's group at the MPI Halle (e.g. [94,95]). The intriguing advantage is that this method gives access to the $k_{\|}$-distribution in the full half-space above the sample surface. Given sufficiently high photon energy, 


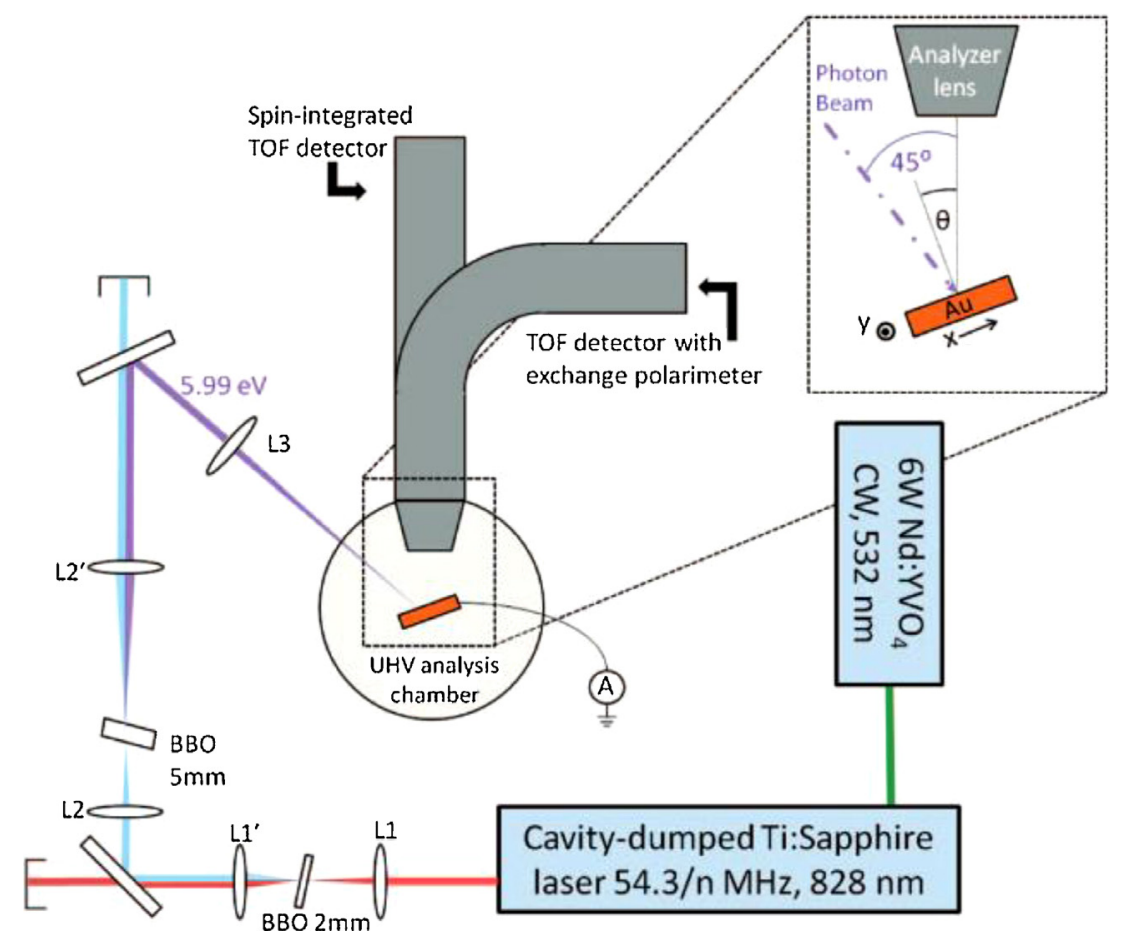

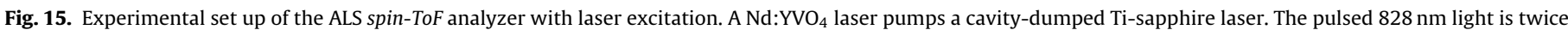

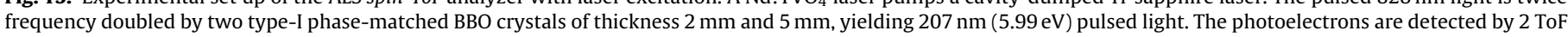
branches, one for spin-integral and one for spin-resolved detection (from [25]).

the half space corresponds to a large momentum range exceeding the first Brillouin zone.

In the instrument described here, an imaging ToF section is implemented for parallel energy acquisition instead of a dispersive analyzer. The detection concept is similar to that of the ToF-PEEM developed at the University of Mainz [28,96]; however, the lens system is optimized for best $k$-resolution. With respect to the $3 D$ data acquisition there are analogies to the ToF spectrometer family of instruments [29,30]. Differences concern the linear $k_{\|}$scale, the large momentum range and high momentum resolution, the large energy range from the threshold to the HAXPES regime, the planar isochronous surfaces in the ToF section and the possibility to implement an imaging spin filter as will be discussed in Section 6.2. Concerning resolution, a benchmark has been set by the instrument at the MPI Halle, where a momentum resolution of $<5 \times 10^{-3} \AA^{-1}$ along with an energy resolution of $12 \mathrm{meV}$ has been reached with the same optical column as used in the ToF instrument [22,23].

The time-of-flight momentum microscope increases the degree of parallelization by replacing the dispersive (i.e. bandpass-type) analyzer by parallel energy detection via ToF. This constitutes a $3 D$-method for $k$-space mapping with great efficiency. The heart of the experiment is the $3 D\left(k_{x}, k_{y}, t\right)$-resolving DLD [96-98]. The ToF method of energy discrimination bears the advantage that many energy surfaces are acquired simultaneously. It requires pulsed photon sources (e.g. synchrotron or FEL radiation, lasers, or highharmonic sources). Alternatively, an electron-optical chopper can be employed together with cw excitation.

Fig. 17 shows a schematic cross section of the ToF $k$-microscope with trajectories for $100 \mathrm{eV}$ start energy and a drift energy of $30 \mathrm{eV}$ in the ToF section. The first momentum image is located in the backfocal plane (BFP) of the objective lens. A size-selectable and adjustable field aperture is located in the first Gaussian image plane. It defines the source spot on the sample surface in a wide range from a few $100 \mu \mathrm{m}$ down to the region of $<1 \mu \mathrm{m}$, independent of the focusing quality of the photon source. The selected source spot in (a)

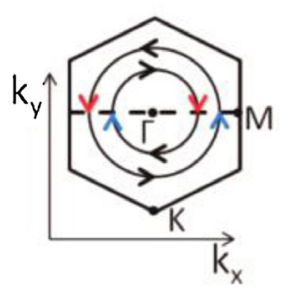

(b)

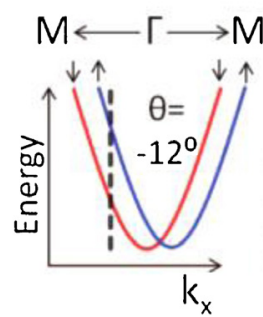

(c)

Acquisition time:

\section{3 minutes}

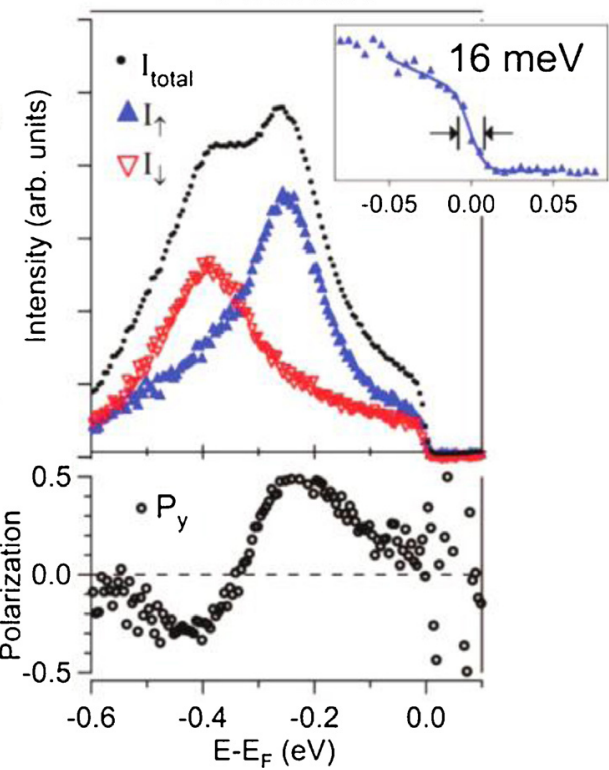

Fig. 16. Shockley surface state of $\mathrm{Au}(111)$ along $\Gamma-M$, measured with the ALS spin-ToF analyzer. (a) Fermi surface diagram showing the spin-orbit split Shockley surface state around $\Gamma$, with its marked Rashba-type spin texture denoted by arrows. (b) Spin-up (blue) and spin-down band (red) in the energy-momentum section along $\Gamma-M$. (c) Spin-integrated and spin-resolved photoelectron spectra, again color coded taken at fixed emission angle $\left(\theta=-12^{\circ}\right)$, denoted by the vertical dotted line in (b). The corresponding spin polarization profile is shown in the lower panel, the inset top right shows the detail close to the Fermi edge (from [25]). 


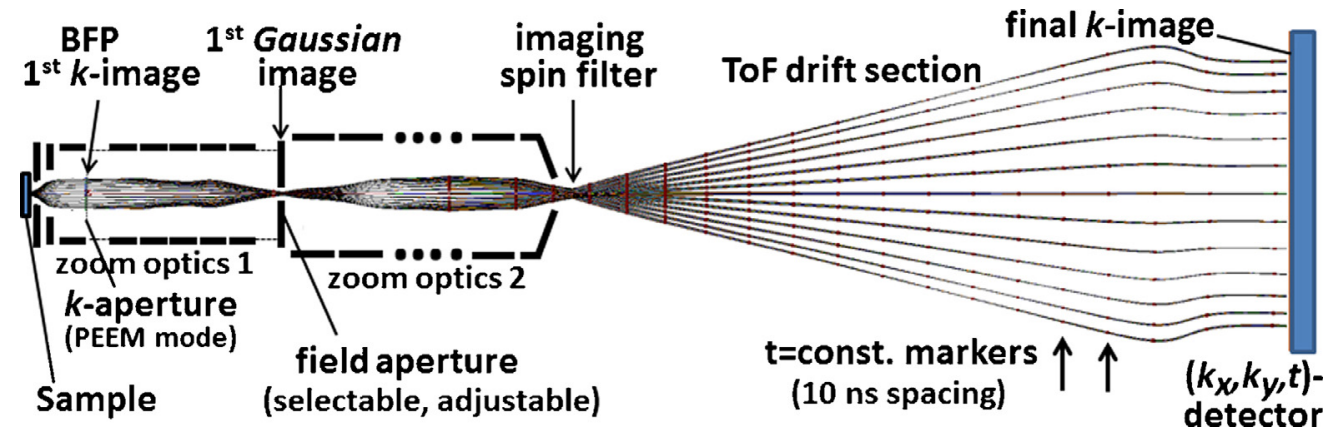

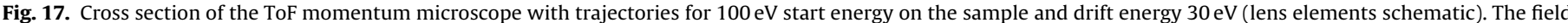

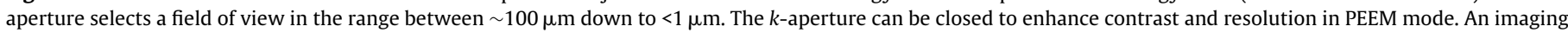
spin filter can be inserted as indicated. Note the planar isochronous contours in the ToF section, marked by dots. Radial coordinate is stretched by $10: 1$.

the Gaussian image acts in the same way as the usual contrast aperture, placed in a reciprocal image plane of a conventional real-space photoelectron microscope. For sufficiently high photon flux density on the sample, a field of view down to the sub-micrometer range can be selected, at the expense of a smaller accessible momentum disc. Electron-optical selection of a small source spot is advantageous for inhomogeneous samples, especially because in this instrument a full-field PEEM image can be inspected before putting the selection aperture to the desired place.

A zoom lens transforms the electron beam to the desired drift energy in the imaging ToF spectrometer. Photoelectron momentum maps (i.e. the $E$-vs- $k$ spectral functions) are taken simultaneously in an energy interval of several $\mathrm{eV}$ width, limited by the chromatic aberration of the instrument. For a length of the drift section of $900 \mathrm{~mm}$ and a time resolution of the DLD of $150 \mathrm{ps}$, the energy dispersion is $\Delta E / \mathrm{meV}=0.255\left(E_{d} / \mathrm{eV}\right)^{3 / 2}$ [99]. Drift energies $E_{d}$ between $30 \mathrm{eV}$ and $4 \mathrm{eV}$ thus lead to theoretical energy resolutions of $\Delta E=40 \mathrm{meV}$ down to $2 \mathrm{meV}$, respectively. Presently a resolution of $19 \mathrm{meV}$ has been achieved, along with a momentum resolution of $0.01 \AA^{-1}$. The DLD has an active area of $40 \mathrm{~mm}$ diameter and a spatial resolution of $80 \mu \mathrm{m}$, resolving 200,000 image points (more than can be transmitted by the electron optics). The maximum integral count rate is 8 Mcps.

The zoom optics can also be used to focus a Gaussian image onto the DLD for spectroscopic ToF-PEEM imaging. In this mode a $k$ aperture is moved into the BFP, acting as normal contrast aperture for resolution optimization. Both apertures are driven by piezomotors with absolute position readout. The crossover behind the zoom optics is the place for an optional imaging spin filter, cf. Section 6.2.

A special property of the ToF momentum microscope is the small distortion of the isochronous surfaces. The dots at each trajectory denote time markers with $10 \mathrm{~ns}$ spacing, which are located on (almost) planar surfaces as visible until the end of the drift section. In other words, there is essentially no correlation between the transversal momentum and the kinetic energy. This is an outstanding feature of the present instrument, different from other spectroscopic ToF devices. There are two reasons for this: (i) the cathode lens is a strong immersion lens causing a virtual image of the source at apparently much higher start energy of $\sim 20 \mathrm{keV}$ (depending on the extractor potential) and with a much smaller cone of effective emission angles; (ii) in the subsequent electronmicroscope column there is no significant beam deterioration (small filling factors of lenses, low aberrations). The radial coordinate in Fig. 17 is $10 \times$ stretched, i.e. in an isogonic plot all rays would run very close to the axis except for the linearly diverging beam in the drift section.

The principle of data acquisition is illustrated in Fig. 18(a). All counting events in the $E-k_{\|}$paraboloid confined by the constant energy surface at the Fermi energy $E_{F}$ and the photoemission
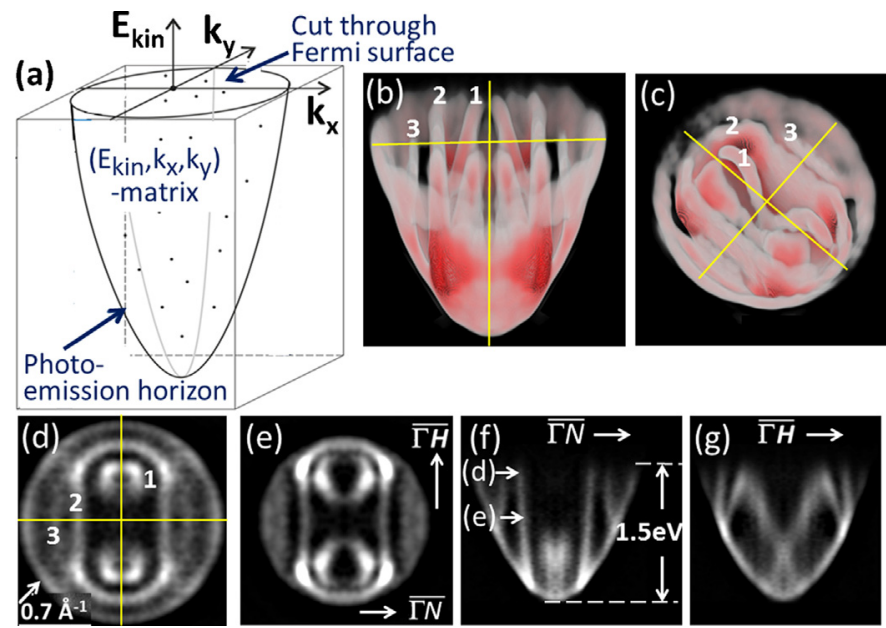

Fig. 18. $3 D$ data acquisition principle of a ToF $k$-microscope: (a) schematic view of the $\left(E_{\mathrm{kin}}, k_{x}, k_{y}\right)$ data matrix confined by the parabolic photoemission horizon and the Fermi energy. (b) and (c), perspective views of the as-measured surface band structure (spectral function $E_{B}$ vs $\left(k_{x}, k_{y}\right)$ ) of an oxygen-covered $\mathrm{Mo}(110)$ surface taken using fs-laser radiation. Bottom row: $\left(k_{x}, k_{y}\right)$-sections close to the Fermi energy (d) and $0.65 \mathrm{eV}$ below $E_{F}(\mathrm{e})$. (f) and $(\mathrm{g}),\left(E_{B}, k_{\|}\right)$-sections along two high-symmetry directions. Data were taken with 2PPE excitation at $h v=3.3 \mathrm{eV}$ (2nd harmonic of a Ti-sapphire laser oscillator); acquisition time $8 \mathrm{~min}$.

horizon (condition $k_{\perp}=0$, means $\theta=90^{\circ}$ ) are detected at fixed setting of the $k$-microscope. The binding energy (with respect to $E_{F}$ ) is determined as in classical photoemission $\left(E_{B}=h v-E_{\text {kin }}-\Phi ; \Phi\right.$ work function) utilizing the relation $E_{\text {kin }}=0.5 m_{e}(l / \tau)^{2}\left(l, \tau\right.$ and $m_{e}$ being the length of the drift section, the time of flight and the electron mass, respectively). The DLD accumulates counting events in the $\left(k_{x}, k_{y}, E_{B}\right)$-voxels of the data matrix. For visualization either the full 3D object is displayed (as in Figs. 18((b) and (c)) and 19((a)-(c))), or sections can be cut in any plane (as in Figs. 18(d-g), Fig. 20 and Fig. 21).

For the first experiments a frequency-doubled Ti-sapphire laser was used for $2 \mathrm{PPE}$ excitation at $2 h v=5.8-6.6 \mathrm{eV}$. The laser oscillator runs at $80 \mathrm{MHz}$, so the period is rather small $(12.5 \mathrm{~ns})$. The width of the data matrix in Fig. 18 is $1.5 \mathrm{eV}$. With the time resolution of 150 ps of the DLD a maximum of 80 energy points can be resolved. Mapping a complete data matrix with good statistics requires only few minutes of acquisition time. The example in Fig. 18((b) and (c)) shows two perspective views of the surface band structure for an oxygen-covered $\mathrm{Mo}(110)$ surface (prepared by heating the clean $\mathrm{Mo}(110)$-surface in $10^{-7}$ mbar of oxygen for $20 \mathrm{~min}$ ). The spatial plots under different perspectives show the raw $\left(k_{x}, k_{y}, \tau\right)$ data without any treatment (except setting of the image contrast), retaining the linear time axis. The time scale translates into an 

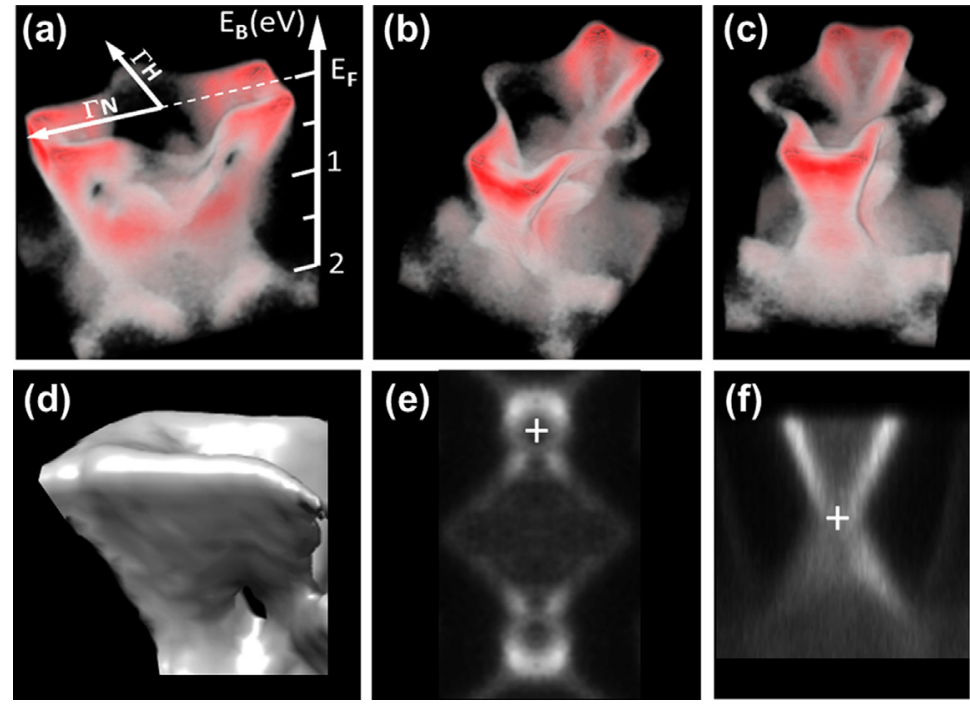

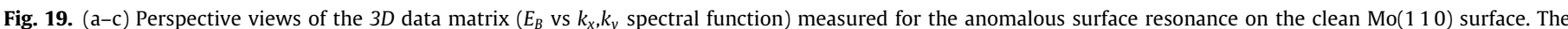

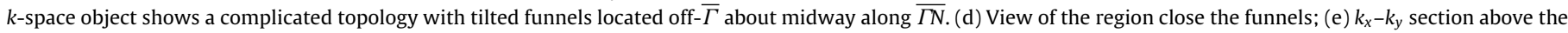

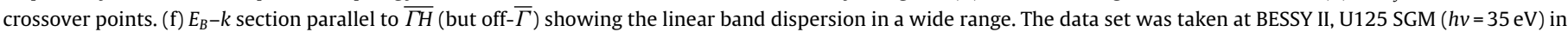
single-bunch mode at a bunch period of $800 \mathrm{~ns}$ (after [100]).

energy scale according to the relation $E_{\text {kin }} / \mathrm{eV}=[1567 \mathrm{~ns} / \tau]^{2}$ (valid for $900 \mathrm{~mm}$ drift section of the present instrument). The $E_{B}\left(k_{x}, k_{y}\right)$ plot reveal three bands with a special dispersion behavior; especially band 1 is tilted in $k_{x}$ and $k_{y}$ direction and does not run in one of the high-symmetry planes in $k$-space. The linear dichroism asymmetries are eliminated in these plots (see below). Except for a crossing point at about $1 \mathrm{eV}$ below $\mathrm{E}_{\mathrm{F}}$, none of the bands runs through the $\bar{\Gamma}$-point as is visible in the momentum discs (d,e). The sections in the canonical high-symmetry directions (f,g) cannot reveal the correct $3 D$ structure. The advantage of rapid $3 D$ data acquisition is clearly evident in this example.

The energy- and $k$-resolution were determined as $19 \mathrm{meV}$ and $10^{-2} \AA^{-1}$, respectively (both a factor of 2 larger than the theoretical values). For the Ti-sapphire experiment (energy interval $1.5 \mathrm{eV}$, diameter of observed $k_{\|}$-disc $1.4 \AA^{-1}$ ), we arrive at a number of several $10^{3}$ resolved $k$-points in the topmost momentum disc and about 75 energy intervals yielding $\sim 10^{5}\left(k_{x}, k_{y}, E_{B}\right)$-voxels acquired simultaneously in the " $k$-space object" shown in Fig. 18. In the first experiment with synchrotron radiation (next section) the $k_{\|}$-range was increased to $3.4 \AA^{-1}$ diameter, yielding $>10^{5}$ voxels. Low sample temperature and better noise elimination will drive the energy resolution to $<10 \mathrm{meV}$ in an interval of $1.5 \mathrm{eV}$ width (limited by the chromatic aberration of the electron optics). This suggests a potential of $\sim 10^{6}$ voxels, being much higher than the values reached in $E-\theta$ mode of a conventional electron analyzer. The advantages will be of particular importance for experiments at low cross section (e.g. in the HAXPES range), for time-resolved studies and for reactive or radiation-sensitive samples.

Let us consider the required time structure of the photon source for the case of $10 \mathrm{meV}$ resolution in more detail: Given the energydispersion value stated above, $10 \mathrm{meV}$ resolution requires a drift energy of $12 \mathrm{eV}$, the DLD resolution is $150 \mathrm{ps}$ and let us assume a width of the transmitted spectrum of $6 \mathrm{eV}$. Then an "ideal" period would be in the range of $200-500 \mathrm{~ns}(2-5 \mathrm{MHz}$ pulse rate). The electron optics allows for effective suppression of slow electrons. Due to the space charge the total number of acceptable counts per pulse is limited, so acquisition time for a $3 D$ data matrix is proportional to the pulse rate. The DLD allows count rates of up to $8 \mathrm{Mcps}$, on average $<1$ electron per photon pulse. A strategy for the correction of part of the space-charge interaction is discussed in Section 6.1. When an imaging spin filter is implemented and operated at the low-energy working point (scattering energy around $11 \mathrm{eV}$ ) it acts

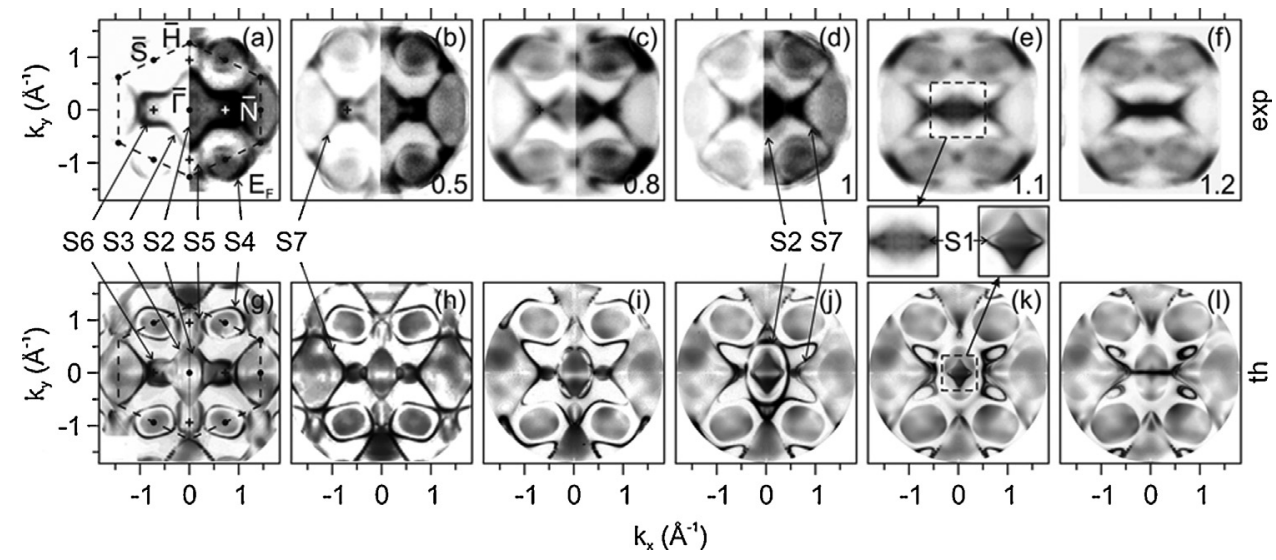

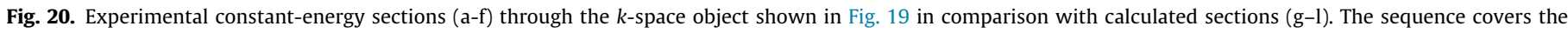

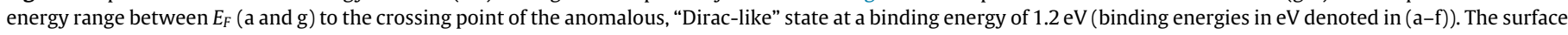
states and resonances are denoted by S1-S7, (a-d) are shown with two different contrast settings, the details of (e) and (k) show the center region (after [100]). 

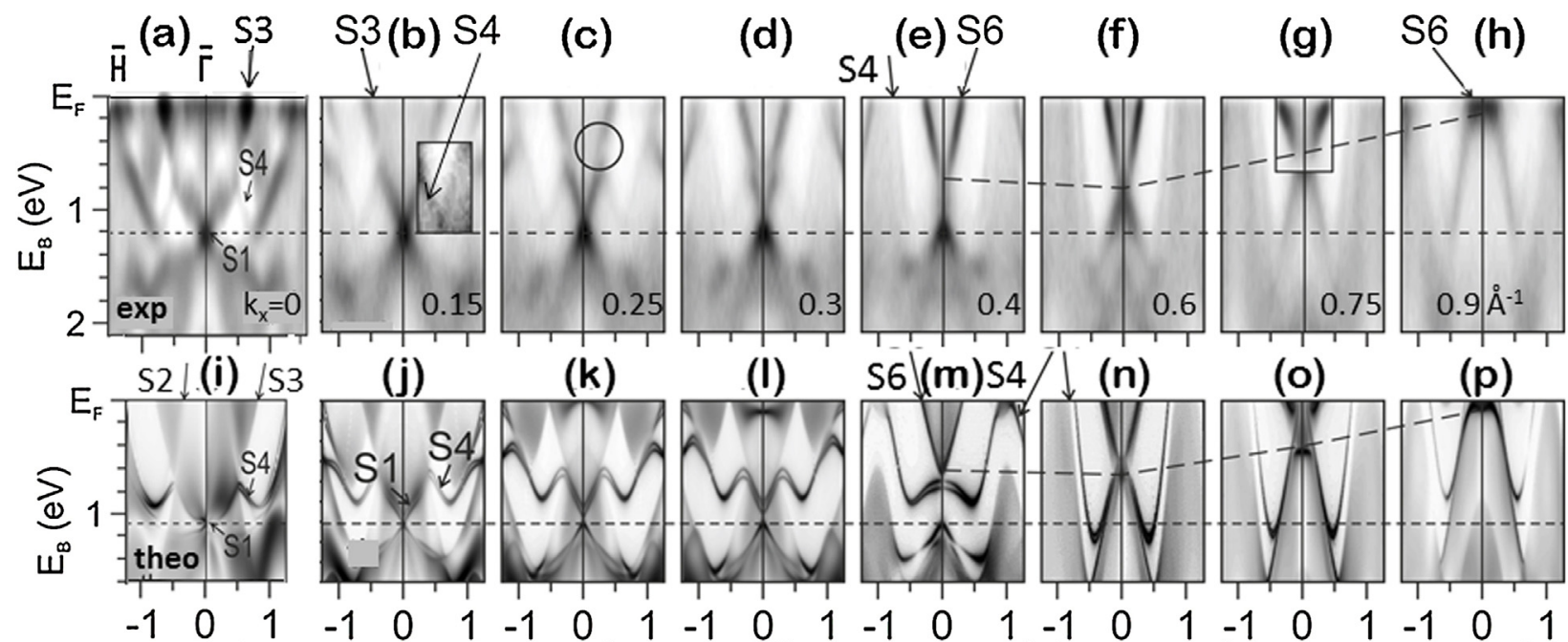

(o)
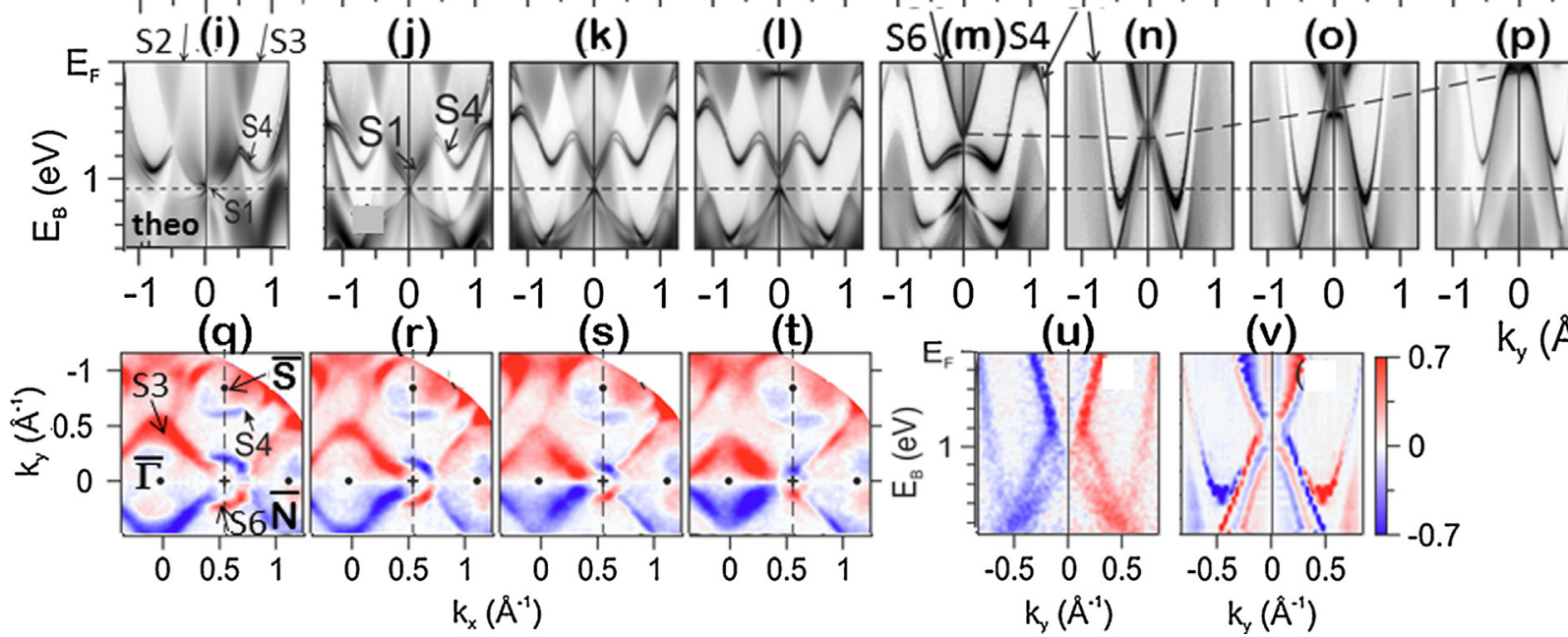

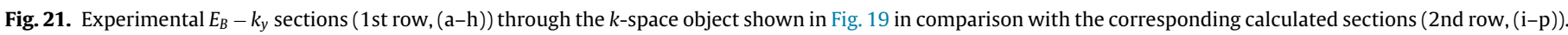

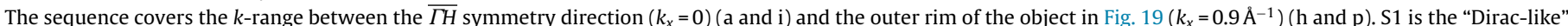

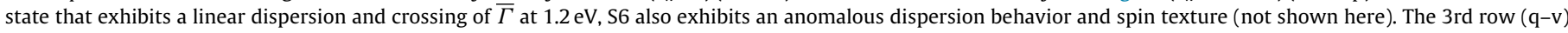

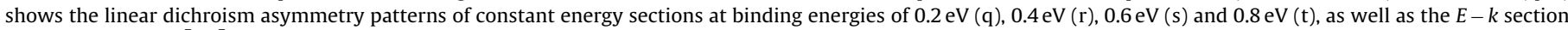
along the direction $\bar{S}-\bar{S}(\mathrm{u})$ compared to theory (v). The circle in (c) denotes a hybridization gap (after [100]).

as additional high-pass filter; if it is operated at the $40 \mathrm{eV}$ working point, the secondary electrons from the spinfilter crystal are cut off by the low ToF energy.

Since the ToF $k$-microscope is an electron microscope with small intermediate images in real space and $k$-space and zoom optics in between, it is straightforward to implement an electron-optical bunch selector. This can be used as "pulse-picker" for laser and synchrotron excitation where it e.g. transmits the hybrid bunch and blanks all others. This solution makes the microscope versatile because it can be used in multi-bunch operation (with full radiation load, however, because the selector acts on the electrons and not on the photons). The possibility of triggering an electronic pulse generator with the synchrotron bunch marker at a small jitter in the $10 \mathrm{ps}$ regime was proven previously [34]. Presently, strategies of chopping the continuous signal from a cw source (by a deflector/aperture assembly upstream of the ToF section) are tested.

In conclusion, ToF $k$-microscopy provides a direct look into the $E$-vs- $k_{||}$spectral function on a linear $k_{||}$-scale with very high energy and momentum resolution in a range exceeding the first surface Brillouin zone. Parallel energy acquisition of several $\mathrm{eV}$ is facilitated by implementation of an imaging ToF spectrometer. The $3 D$ data acquisition causes minimum radiation damage to delicate samples, because all electrons from the source spot (with size and position being defined by a field aperture in the column) are detected in parallel with a transmission factor of 1 .

\subsection{D mapping of the electronic structure}

One of the first systems studied with the ToF $k$-microscope was the Mo(110)-surface [100], which has a surface band structure similar to that of W(1 1 0) [101]. For W(1 1 0), an anomalous surface resonance in a spin-orbit induced partial band gap was recently discovered by Miyamoto et al. [102,103]. It is characterized by a band crossing and linear dispersion in a large energy range, resembling the Dirac state of a topological insulator. The study was motivated by the question whether the similarity of Mo and W extends to the existence of the anomalous surface resonance. Since the spin-orbit interaction in Mo is 5 times smaller than in $\mathrm{W}$, the existence of this special state on the Mo(110) surface was an open question [104].

Measurements using synchrotron radiation were performed in single-bunch mode at BESSY at the beamline U125-2 SGM [105] at a photon energy of $35 \mathrm{eV}$. Given the work function of $\mathrm{Mo}(110)$ of $4.95 \mathrm{eV}$ [106] this results in a kinetic-energy range of $30 \mathrm{eV}$ width with the lowest $15 \mathrm{eV}$ being cut off by the transfer lens to the ToF section. Typical photon flux at $35 \mathrm{eV}$ was of the order of $10^{10}$ photons per second for the single-bunch conditions of $<10 \mathrm{~mA}$. The photon spot was $150 \times 80 \mu \mathrm{m}^{2}$ in size, the actual source size $(80 \mu \mathrm{m}$ dia. for the measurements shown in this section) was defined electron optically by the field aperture, $\mathrm{cf}$. Fig. 17 . The photon beam was linearly $p$-polarized in horizontal direction (the E-vector forming an angle of $22^{\circ}$ with the surface normal) and the azimuth of photon impact could be varied via sample rotation.

Fig. 19((a)-(d)) shows perspective views of the $3 D$ data matrix acquired by the ToF $k$-microscope for the anomalous surface resonance on $\operatorname{Mo}(110)$. The surface states and resonances show up as a pronounced " $k$-space object" in the form of a characteristic pattern of an elongated rectangular profile with diamond-shaped central part. The rectangle contracts with increasing binding energy and its outer contour shows a linear dispersion. Compared with a typical two-dimensional Dirac state with a conical shape, the surface resonance in Fig. 19 appears strongly warped by the 2-fold symmetry of the Mo(1 10$)$-surface. Close inspection of the $3 D$-object reveals 
several interesting details of the topology: the linearly-dispersing outermost boundaries of the $k$-object in views (b,c,d), the holes along the $\overline{\Gamma \mathrm{H}}$ direction (a,d), the narrow elongated waist (c), the two funnels (marked by + ) midway along $\overline{\Gamma \mathrm{N}}$ in constant-energy section (e) and section (f) and the onset of bulk bands at the lower end of the displayed energy region appearing as diffuse structures at the lower end of the band gap, $E_{B}<1.4 \mathrm{eV}$. In order to see the inner structure, sections can be cut in all planes. The $k_{x}-k_{y}$ section (e) was cut above the crossover points (at $E_{B}=0.77 \mathrm{eV}$ ) providing a view into the funnels. The $E_{B}-k$ section in (f) has been extracted in a plane Á off- $\bar{\Gamma}$, parallel to the $A \overline{\Gamma H}$ direction. It displays the linear dispersion of the surface resonance over a remarkably large energy range of $800 \mathrm{meV}$. Fig. 19 shows the raw data as measured by the delay-line detector without any data treatment, except setting the contrast in the display program and a symmetrization that eliminates the linear dichroism (see below).

Acquisition took $20 \mathrm{~min}$ for this data set (at the non-ideal bunch period of $800 \mathrm{~ns}$ ). The diameter of the observed momentum disc was set to $3.4 \AA^{-1}$ at an estimated resolution of $0.02 \AA^{-1}$. This results in $\sim 10^{4}$ resolved $k$-points in one momentum disc. Given the rather low energy resolution during the first beamtime (about $80 \mathrm{meV}$ ) along with a well-focused energy interval of $1.5 \mathrm{eV}$ (limited by chromatic aberration of the electron optics), the data set of Fig. 19 resolves some $10^{5}$ data voxels. For the present resolution of $<20 \mathrm{meV}$ this value increases to the order of $10^{6}$ voxels. These are acquired simultaneously without energy sweep, change of focusing or movement of angles.

In order to compare the data with a calculation (cf. [100]), we cut sections through the $3 D$ data matrix. Fig. 20 shows the comparison of experimental (1st row) and theoretical (2nd row) constant energy sections between the Fermi energy $(\mathrm{a}, \mathrm{g})$ and the pronounced crossover line along $\overline{\Gamma N}$ at a binding energy of $E_{B}=1.2 \mathrm{eV}(\mathrm{f}, \mathrm{l})$. The surface Brillouin zone (SBZ) is marked by the dashed line in (a,g) and high-symmetry points as well as surface states and resonances S1-S7 are denoted. There is good overall agreement between experiment and theory. The oval feature around the $\bar{S}$-point, the eye-catching diamond-shaped pattern centered at $\bar{\Gamma}$ and the adjacent rectangular (horseshoe-shaped) features have been observed before [101]. They are denoted as S3 and S6, respectively. These bands have a different symmetry character, as evident from measurement of the linear dichroism, see panels $(q-t)$ in Fig. 21 and [100]. The surface resonance enclosing symmetry point $\bar{N}$ and the two bands running towards the $\bar{H}$-point are labeled as S5 and S7, respectively. Being surface resonances these states derive from bulk bands. The oval narrow bands S4 and S2 surrounding the $\bar{S}$-points and the $\bar{\Gamma}$ - point (see (d,j) in Fig. 20) are surface states; they disappear rapidly upon residual gas adsorption. The most interesting state is the anomalous band S1 that strongly resembles a Diracstate in a topological insulator. It shows a crossover line at $1.2 \mathrm{eV}$ (f,l) and has an elliptical constant-energy section slightly above the crossover (cf. details of $(\mathrm{e}, \mathrm{k})$ ).

The band dispersions can be visualized by cutting $E_{B}$-vs- $k$ sections along any orientation in $k$-space, along high-symmetry directions but also off- $\bar{\Gamma}$. For $\operatorname{Mo}\left(\begin{array}{lll}1 & 10\end{array}\right)$ such sections revealed a similar topology of the anomalous band S1, also termed "Diraclike" similar as observed for W(110) [102]: a flat band along the $\overline{\Gamma N}$-direction and a band crossing with linear dispersion along $\overline{\Gamma H}$ and $\overline{\Gamma S}$ [100]. Fig. 21 shows a sequence of experimental (a-h) and theoretical sections (i-p) in the $\overline{\Gamma H}$ symmetry plane (a,i) and parallel to that plane with increasing distance from the $\bar{\Gamma}$-point between $k_{x}=0.15 \AA^{-1}(\mathrm{~b}, \mathrm{j})$ and $0.9 \AA^{-1}(\mathrm{~h}, \mathrm{p})$.

At the $\bar{\Gamma}$-point the band gap is only $120 \mathrm{meV}$ wide, hence the "Dirac-like" band hybridizes with bulk states in the paraboloidshaped bulk continuum confined by S3 (a,i). However, as little as $0.15 \AA^{-1}$ away from $\bar{\Gamma}(\mathrm{b}, \mathrm{j})$ the bulk continuum is retracted, the gap is larger and S1 clearly exhibits its linear dispersion until
$0.3 \AA^{-1}(\mathrm{~d}, \mathrm{l})$. At $0.4 \AA^{-1}$ (e,m) S1 disappeared and the horseshoeshaped band $S 6$ appears as an intense $\mathrm{V}$-shaped feature close to $E_{F}$. S6 exhibits a linear dispersion and crossing point, too, which is located at $E_{B}=0.77 \mathrm{eV}(\mathrm{f}, \mathrm{n})$. Sections (h,p) run along the outer rim of the horse-shoe, cf. Fig. 19((b)-(d)). S6 is completely located in the bulk band gap (white area in (e-g)) and appears as a tilted funnel in Fig. 19((a) and (d)). The linear dispersion of the walls of this funnel and an unusual spin texture revealed by the calculation (not shown here) give evidence of the anomalous nature of this state, with the crossing point being located off- $\bar{\Gamma}$. The agreement with theory is generally very good, except for the relative intensities. In particular, band S4 appears very intense in theory $(\mathrm{i}-\mathrm{n})$, whereas it is rather weak (though significant) in experiment $(\mathrm{a}-\mathrm{c})$.

The panels in the bottom row of Fig. 21 show the linear dichroism in the angular distribution of photoelectrons (LDAD) as discussed (together with the related circular dichroism CDAD) in [107-109]. The LDAD is derived from the intensity differences in the two half planes (here $k_{y}>0$ and $k_{y}<0$ ) as described in [100]. The LDAD asymmetry values in the $k_{x}-k_{y}$ maps $\left((\mathrm{q}-\mathrm{t})\right.$ for $E_{B}=0.2$, $0.4,0.6$ and $0.8 \mathrm{eV}$, respectively) and the $E_{B}-k_{y}$ sections $(\mathrm{u}, \mathrm{v})$ are color coded as defined by the scale bar in (v). The asymmetry shows a rich bipolar structure in the sections and reaches values as high as $\pm 70 \%$. In particular it reveals that surface bands S3 and S6 have different symmetry. Without going into detail we mention that the LDAD (like CDAD) gives information about the non-relativistic single-group symmetries (orbital character) of the band states. Hence, these non-magnetic dichroism quantities complement spin-resolved measurements that give access to the relativistic double-group symmetries. There is good overall agreement between experiment and theory in intensity and dichroism, with some significant differences in details, cf. [100].

Diagrams like Figs. 20 and 21, i.e. $k_{x}-k_{y}$ or $E_{B}-k_{\|}$sections are the typical representations of electronic band structures. In the new experimental approach of ToF $k$-microscopy they are generated from the full $3 D$ matrix by just extracting $2 D$ sub-stacks. The examples in Figs. 18 and 19 demonstrate that the $3 D$ acquisition of the $E_{B}$-vs- $k$ spectral function provides the maximum possible information content. The perspective $3 D$ views exhibit tilted band features that are not visible in the conventional sections in highsymmetry planes. In classical spectroscopy such an analysis would require mosaic-like sequences of $E-\theta$ scans as discussed in Section 3 (note that the momentum discs in Fig. 20 correspond to a solid angle cone of $66^{\circ}$ ). All results in Figs. 19-21 have been obtained in a single accumulation of $20 \mathrm{~min}$ with fixed settings.

As a test case for a material with high current interest we have chosen the topological insulator $\mathrm{Bi}_{2} \mathrm{Te}_{3}$, because detailed data with conventional high-resolution ARPES spectrometers exist [110,111]. This material was cleaved in UHV in order to obtain a clean and well-defined surface. Fig. 22 shows a collection of sections through several planes in the vicinity of the Dirac cone. Note that Fig. 22 shows the as-measured results without any data treatment or symmetrization. The measurement using Ti-sapphire laser excitation (2PPE excitation at $h v=3.3 \mathrm{eV}$ ) reveals the Dirac cone, visible as a $\mathrm{v}$-shaped feature on top of panel (a), confined by the parabolic photoemission horizon. The sixfold warping of the Dirac cone and the structure below the crossover can be clearly discerned in the sections in panel (b). The perspective $3 D$ view (c) of the same data matrix reveals the details of the states below the crossover point (the width of the cone itself is overemphasized in (c)). Owing to the high repetition rate of the Ti-sapphire oscillator this $3 D$ data matrix has been taken in $4 \mathrm{~min}$ of total acquisition time with the field aperture selecting a source spot of only $25 \mu \mathrm{m}$ dia. The period of the laser oscillator is $12.5 \mathrm{~ns}$. Given the time resolution of $150 \mathrm{ps}$ of the DLD, 80 energy slices can be resolved at maximum in this mode. 
(a)

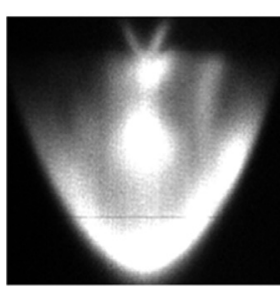

(b)

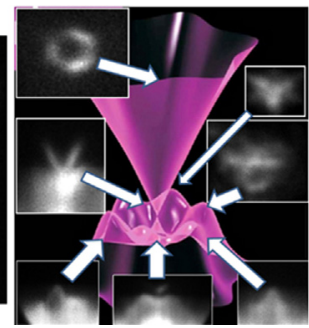

(c)

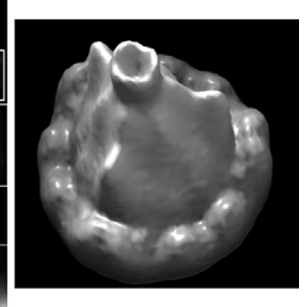

Fig. 22. Result of a first measurement on a sample of the topological insulator $\mathrm{Bi}_{2} \mathrm{Te}_{3}$ taken with 2PPE excitation of a Ti-sapphire oscillator ( $\mathrm{h} v=3.3 \mathrm{eV}, 80 \mathrm{MHz})$. Details of the Dirac state (topological surface state) are resolved in the $E_{B}\left(k_{x}\right)$-representation (a) and several detail sections in (b). The center figure in (b) shows a calculated Dirac state. The perspective $3 D$ view (c) of the same data matrix shows details of the states below the crossover. The figure displays the raw data taken at room temperature in a run of 4 min of acquisition time. (Result from a cooperation with R. Claessen and M. Scholz, Univ. of Wuerzburg, Germany; sample courtesy L.V. Yashina, Moscow State University.).

\subsection{Ultrafast processes in the electronic band structure}

Angular resolved photoemission spectroscopy (ARPES) combined with an ultrafast time resolution is the ideal tool to directly extract dynamical information from the band structure. With effective temporal resolutions of a few $10 \mathrm{fs}$, a pump probe ARPES set-up offers a new perspective on complex materials because the method resolves electron dynamics at or close to the fundamental time scales of electronic processes. The direct dynamical information can be used to temporally discriminate complex phases and identify the dominant degrees of freedom, order parameters, or interactions [112]. Materials in which electron-electron and electron-lattice correlations are strong exhibit some of the most intriguing phenomena in solid state physics, including (hightemperature) superconductivity, metal-insulator transitions, and ordering phenomena involving charge, orbital, spin, and lattice degrees of freedom. The non-equilibrium dynamics of these materials thus provide a very interesting and promising research field.

Time-resolved ARPES has up to now been performed by using laser-based X-ray sources [31]. The pump pulse is typically an infrared $(800 \mathrm{~nm})$ pulse from a Ti-sapphire laser as described in the previous sections. The probe pulses are generated in two steps. The fundamental beam first passes a $200 \mu \mathrm{m}$ thick beta-barium borate (BBO) crystal. The resulting second harmonic is then focused into an argon-filled high-harmonic-generation (HHG) source. The XUV radiation is focused with a gold-coated toroidal mirror to a diameter of about $170 \mu \mathrm{m}$ (FWHM) at the sample position. In the experiments described here the 7 th harmonic $(22.1 \mathrm{eV})$ of the $400 \mathrm{~nm}$ beam was used for the time-resolved measurements. The energy spread of the XUV radiation amounts to $170 \mathrm{meV}$. Typical integration times for spectra are below $10 \mathrm{~s}$. The improvement in comparison to previously used high-harmonic X-ray sources is due to a combination of higher photon conversion efficiency at shorter wavelengths, elimination of monochromatizing optical elements, and less reabsorption in the gas due to a lower phasematching pressure. The photon flux is $10^{11}$ photons/harmonic/ second [31].

A recently published study [113] of time-resolved ARPES investigated the Peierls-Mott insulator $1 T-\mathrm{TaS}_{2}$ and $1 T-\mathrm{TaSe}_{2}$. In these materials the simultaneous occurrence of a charge-density wave and a Mott transition at a critical temperature (180-220 K for $1 T$ $\mathrm{TaS}_{2}$ and $470 \mathrm{~K}$ for $1 T-\mathrm{TaSe}_{2}$ ) indicates a strong relation between the two phenomena. Time- and angle-resolved extreme ultraviolet photoemission spectroscopy is used to determine the momentumdependent electronic structure dynamics on the sub-300 fs time scale, see Fig. 23. Similar studies for the case of $1 T-\mathrm{TiSe}_{2}$ have been reported in $[31,114]$.

The extracted spectroscopic order parameters of the charge density wave and the Mott transition display a global two-time-scale dynamics indicating a quasi-instantaneous loss of the electronic order (less than $30 \mathrm{fs}$ ) and a subsequent coherent suppression of the lattice distortion on a time scale related to the frequency of the charge-density-wave amplitude mode (200-300 fs). After one half-cycle of coherent amplitude-mode vibration, a crossover state between insulator and metal with partially filled-in and partially closed Mott and Peierls gaps is reached. From the spectroscopic results the authors conclude that the bottleneck of the photo-induced melting process of the Peierls-Mott state is the suppression of the characteristic periodic lattice distortion and the fastest way to get through this bottleneck is coherent lattice relaxation along the coordinate of its amplitude mode. The period of the amplitude-mode oscillation thus defines the speed limit of the melting process.

\section{Summary and outlook on current developments}

In this section we try to extrapolate some of the current developments in the field of space-, time- and spin-resolved photoemission into the future. The novel approach of momentum microscopy will be extended into the soft and hard X-ray range, for which we will show results of simulations for different geometries. This method opens up a path towards correction of the deterministic part of space-charge interaction, as verified in a first pilot experiment. Next, we summarize the achievements in $2 \mathrm{D}$ multichannel spin detection in combination with a hemispherical analyser run in the conventional $E-\theta$ acquisition mode (cf. Section 3). We place emphasis on the technical obstacles, in particular those connected with the inherent properties of the electron optics of hemispherical analyzers. The present status is compared with recent results obtained by a momentum microscope using a dispersive analyser, developed at the MPI for Microstructure Physics in Halle. Taking the concept of parallelization further, we discuss the possibility of $3 D$ multichannel spin detection by implementing an imaging spin filter into the ToF momentum microscope introduced in Section 5. Along with the development of high-brilliance light sources in the lab and at large-scale facilities, the electron detector itself can act as the bottleneck in high-throughput experiments. In the last part, the present state and emerging achievements in highly parallelized $3 D$ electron detection are discussed.

\subsection{Momentum microscopy towards higher energies}

Fig. 24 shows calculated trajectories for photoelectrons in a large range of start energies: $5 \mathrm{keV}$ (a), $100 \mathrm{eV}$ (b) and $2 \mathrm{eV}$ (c). For (b) and (c) the full half space was accepted, whereas for (a) the emission cone was reduced to $\pm 45^{\circ}$. In the $5 \mathrm{keV}$ case, as is relevant for bulk-sensitive hard X-ray photoemission (HAXPES), a momentum disc of $25 \AA^{-1}$ radius is visible in the backfocal plane (considerably larger than the acceptance range of conventional HAXPES spectrometers). The challenges of $k$-resolved measurements in the hard X-ray range are discussed by Gray et al. [115]. In the simulated optics, the momentum resolution in the reciprocal image at $5 \mathrm{keV}$ start energy is very high, owing to the optimized cathode lens. $100 \mathrm{eV}$ start energy (b) is at the lower end of the highly interesting soft X-ray range. At this energy, the photoemission horizon (corresponding to the full half space) is $5 \AA^{-1}$, which still comprises several Brillouin zones. Finally, $2 \mathrm{eV}$ start energy (c) corresponds to laser photoemission with typical photon energies of $h v=6 \mathrm{eV}$ (fourth harmonic of a Ti-sapphire laser) or $7 \mathrm{eV}$ (e.g. the third harmonic of a $\mathrm{Nd}: \mathrm{YVO}_{4}(355 \mathrm{~nm})$ 

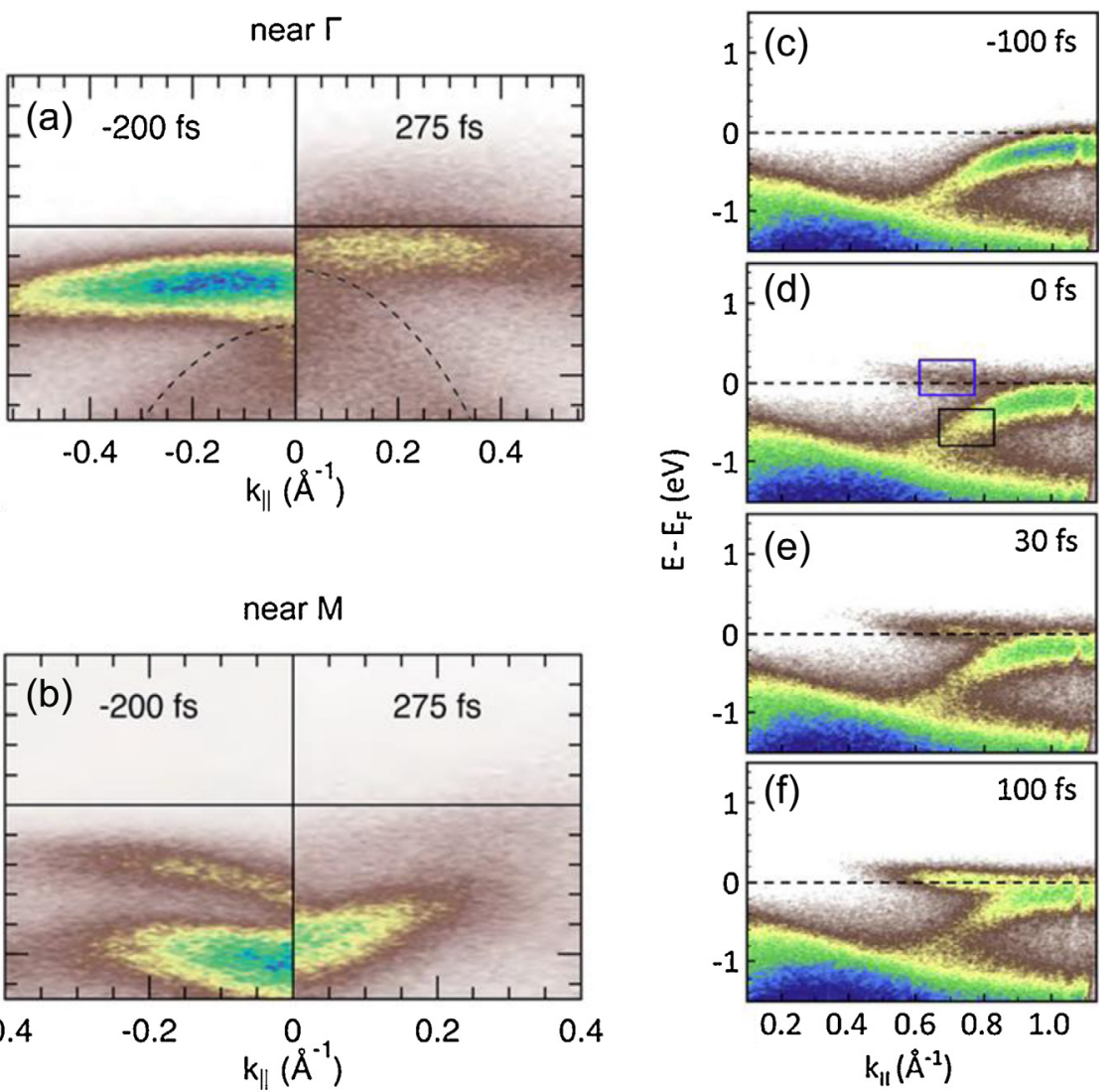

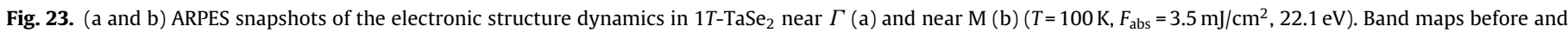

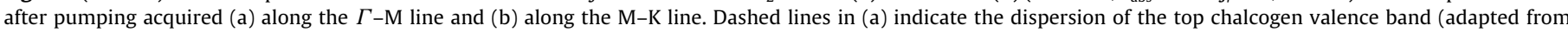

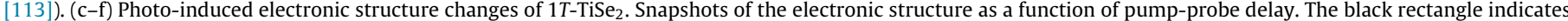

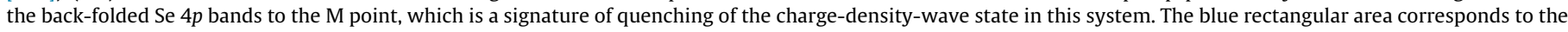
Ti $3 d$ band (adapted from [31]).

frequency-doubled by a KBBF-crystal). For energies this close to the threshold the horizon is as small as $0.7 \AA^{-1}$ radius (examples in Figs. 18 and 22). The simulations (http://simion.com) were done assuming the correct lens geometries (lens elements in
Fig. 24 are schematic). Despite the large energy range, all three trajectory patterns look similar, but panels (b) and (c) have been radially stretched by factors of 5 and 36 with respect to (a), respectively.

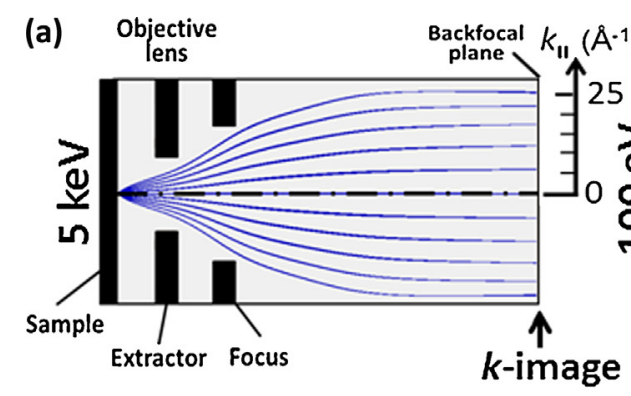

(d)

\section{$k$-image}

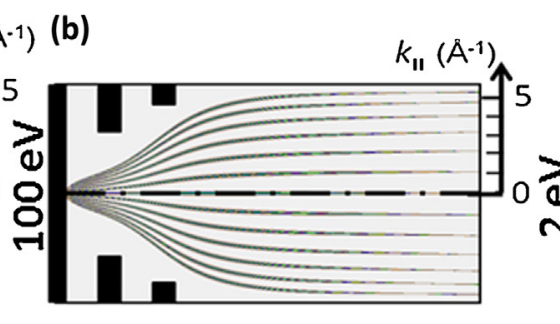

(c)

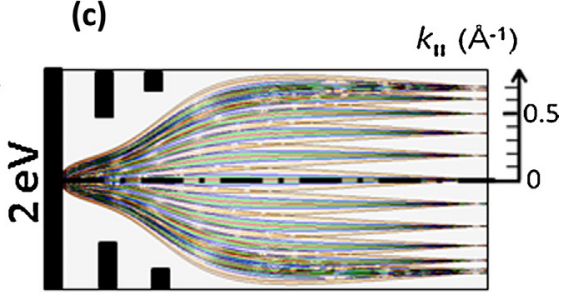

Field aperture

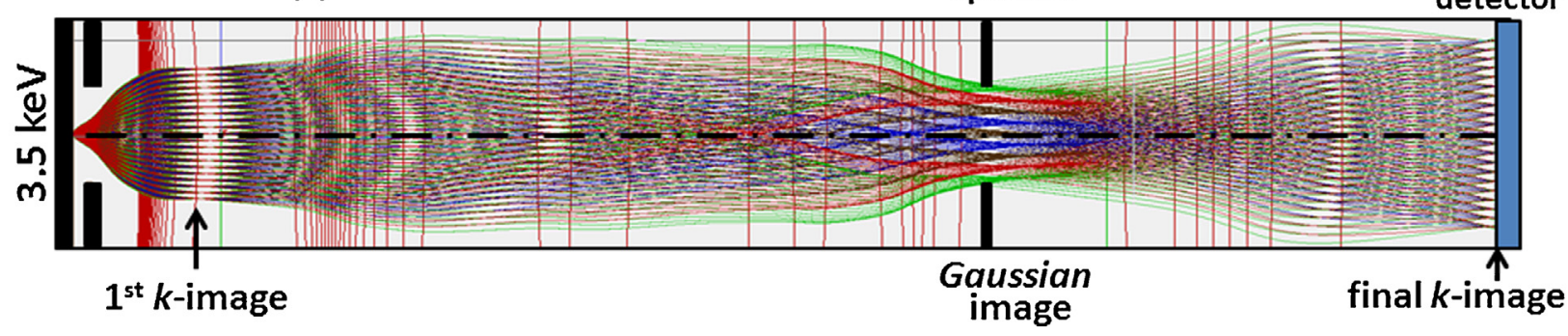

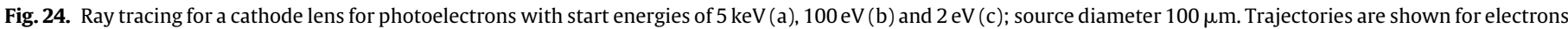

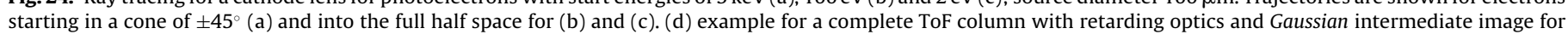
start energy $3.5 \mathrm{keV}$, source spot $400 \mu \mathrm{m}$, drift energy $80 \mathrm{eV}$, final $k$-image at the detector: disc of diameter $10.2 \AA^{-1}$ at high $k$-resolution and low field curvature. 
(a)

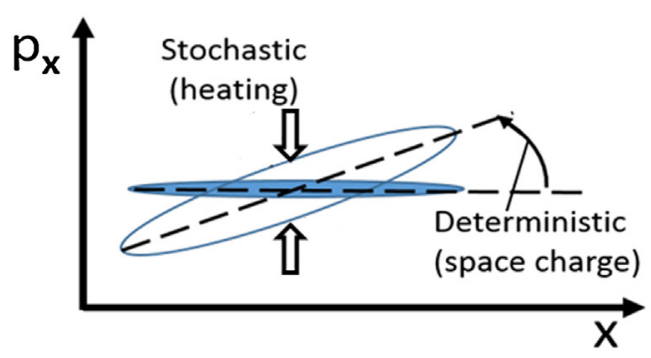

(b)

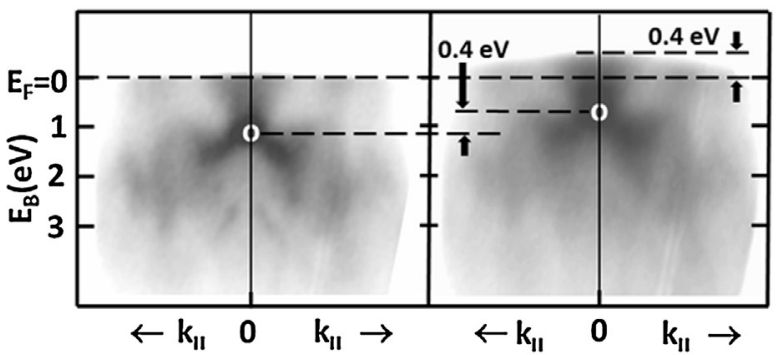

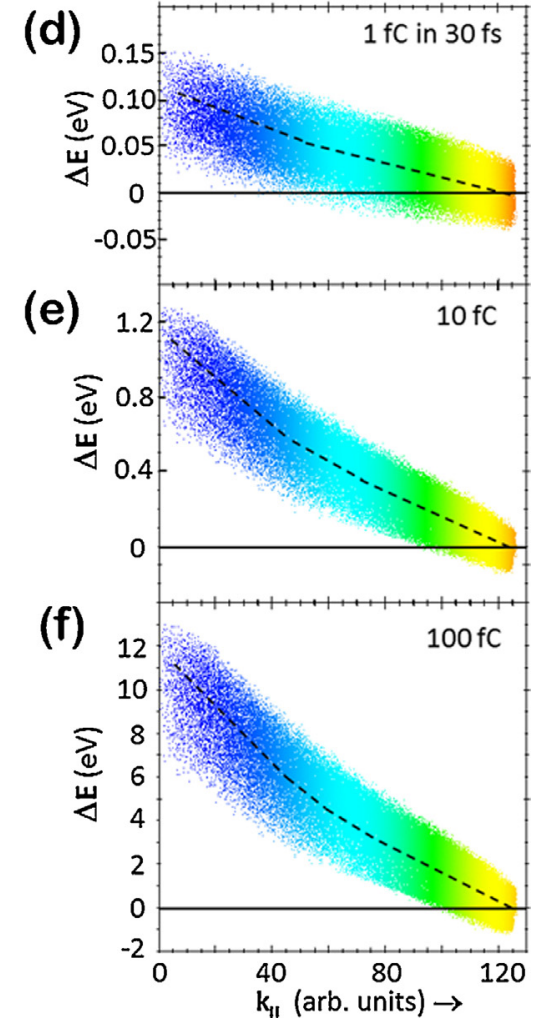

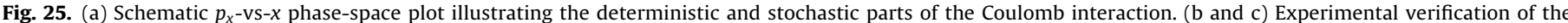

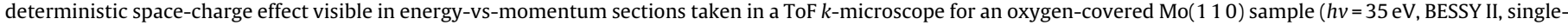

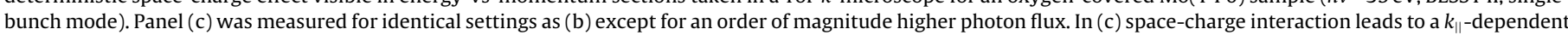

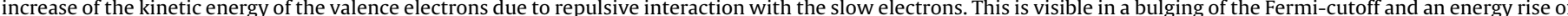

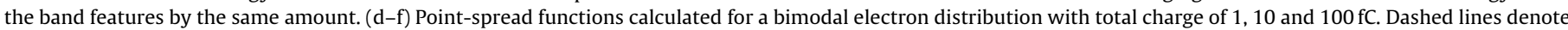
curves of best fit, defining a new $k_{\|}$-dependent energy scale (following [116]).

Panel (d) shows a sample simulation for a complete ToF column with retarding optics and Gaussian intermediate image. Here the start energy was $3.5 \mathrm{keV}$ and the drift energy was $80 \mathrm{eV}$ (as appropriate for HAXPES). The electron-optical system was optimized for high start energies and is different from Fig. 17. A field aperture in the intermediate image can be used to confine the source spot on the sample. A special property of this optical arrangement is that the source region can be very large while the $k$-resolution remains high. The example in Fig. 24(d) was simulated for a source region of $400 \mu \mathrm{m}$ diameter. With the same objective lens and a modified operation mode of the column, the simulation revealed a $k$-resolution of $0.014 \AA^{-1}$ (corresponding to $0.027^{\circ}$ angular resolution at $3.5 \mathrm{keV}$ start energy) for as much as $2 \mathrm{~mm}$ accepted source area! For homogeneous samples, a large source area is advantageous because it strongly reduces radiation damage and space-charge interaction. For small or inhomogeneous samples, the source area can be confined by the field aperture in the Gaussian image as described in Section 5.1.

High-brilliance sources like synchrotrons, free electron lasers or table-top high-harmonic sources pave the way to electron spectroscopy experiments targeting ultrafast dynamics and short-lived transient states. Electron bunches released on a sub-picosecond timescale experience strong Coulomb interactions between emitted electrons resulting in large energy shifts and severe broadening of photoemission signals. The Coulomb interaction contains a deterministic part (usually termed space-charge interaction) and a stochastic part (leading to "heating" of the beam). The interaction of a given electron with the average charge density of all surrounding electrons leads to a rotation of the electron distribution in $6 D$ phase space, as illustrated for one space coordinate $(x)$ and the conjugate momentum coordinate $\left(p_{x}\right)$ in Fig. 25(a). This rotation reflects the fact that an electron at the leading edge of the cloud is accelerated ( $p_{x}$ increases) by the repulsive interaction with all other electrons. Conversely, an electron at the trailing edge experiences an overall deceleration ( $\mathrm{p}_{\mathrm{x}}$ is reduced). This is the deterministic aspect. However, in addition, individual electron-electron scattering processes lead to stochastic (irreversible) heating of the electron ensemble, manifesting itself in a vertical broadening of the distribution. Momentum microscopy gives direct access to the three momentum coordinates, opening a path for a correction of the deterministic part of the Coulomb interaction (space-charge interaction) [116]. Further details on the space-charge problem in FEL experiments are discussed in [117].

In a first experiment with a ToF $k$-microscope using synchrotron radiation at BESSY, the rotation in phase space became directly visible as shown in Fig. 25(b and c). The two energy-vs-momentum sections have been taken at photon fluxes differing by about one order of magnitude. The pattern at lower flux (b) exhibits a sharp, straight cutoff at maximum energy, corresponding to the Fermi edge $E_{F}$. At the higher photon flux (c), a marked deformation of the Fermi cutoff is evident. The energy shift reaches its maximum of $0.4 \mathrm{eV}$ on-axis $\left(k_{\|}=0\right)$ and drops off to both sides with increasing $k_{\|}$. The band features close to $k_{\|}=0$ are lifted in energy by the same amount, the high-intensity point at $k_{\|}=0$ is marked by a circle. In addition, all features are smeared out in panel (c) due to the e-e scattering processes. The parameters of these measurements were: photon energy $35 \mathrm{eV}$, single bunch $(1.25 \mathrm{MHz}$ repetition frequency), photon flux about $10^{10}$ photons/s. The patterns represent the surface state of a Mo(110) sample with oxygen overlayer.

Simulations using the GPT code $[118,119]$ revealed that under realistic conditions, an order of magnitude gain in resolution can be achieved when the space-charge shift is properly corrected for 

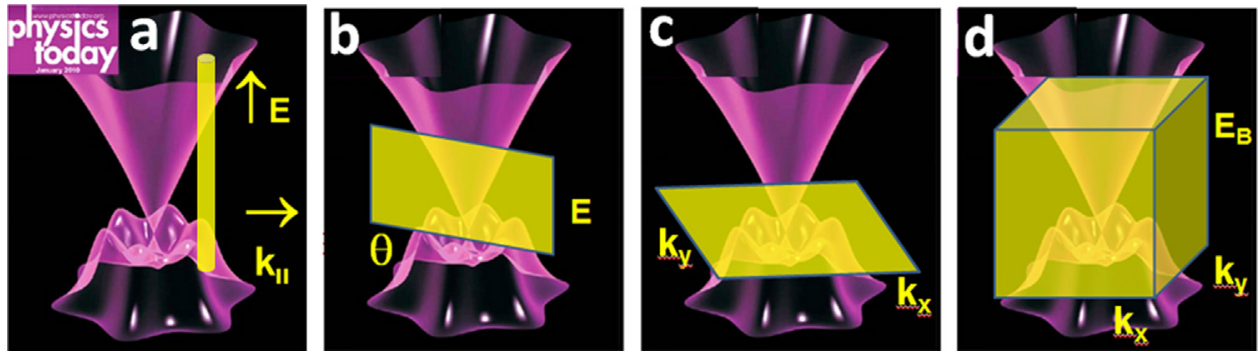

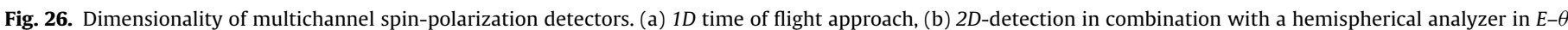

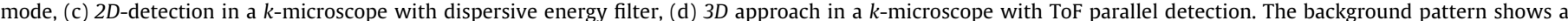
calculated topological surface state in $E_{B}\left(k_{x}, k_{y}\right)$ representation (from Physics Today, vol. 63, issue 1, January 2010).

[116]. The simulation Fig. 25((d)-(f)) assumes a bimodal distribution, with $10 \%$ electrons at a kinetic energy of $5 \mathrm{keV}$ (representing electrons from the valence band) and 90\% at low energy (representing the large signal of secondary electrons). The random distribution was launched within a short time from a small spot (Gaussian temporal and spatial beam profiles of widths $30 \mathrm{fs}$ and $100 \mu \mathrm{m}$ FWHM). The strong immersion field of the cathode lens causes a spatial confinement of the slow electrons into a thin pencil beam travelling along the optical axis. The interaction of the large number of secondary electrons travelling on axis with the rapidly expanding momentum-image disc of the fast electrons is much weaker at large $k_{\|}$than in the central region. This leads to a tilted point spread distribution in the $\Delta E\left(k_{\|}\right)$representation. The broadening of the point distribution in perpendicular direction reflects the stochastic processes. Simulated results for total charges of 1,10 and $100 \mathrm{pC}$ are shown in Fig. 25((d)-(f)); where only the fast electrons are shown. As expected, the size of the energy shift increases linearly with the total charge, released within $30 \mathrm{fs}$. The dashed lines denote best-fits of the point-spread functions and can be used for a renormalization of the energy scales. Since a substantial part of the Coulomb interaction is deterministic, it can be partially corrected for in a momentum microscope (for details, see [116]). In common ARPES or ToF spectrometers, the secondary electrons fly along with the fast electrons in all directions. Therefore, the rapid radial separation of the momentum discs of slow and fast electrons does not occur and the space-charge interaction is about the same in all emission directions. Only a temporal separation due to the different velocities exists.

\subsection{Future of multichannel spin detection: Prospects and obstacles}

Multichannel spin detection exploits the fact that in lowenergy electron diffraction, $k_{\|}$is conserved, as is the case for an optical mirror. With $\operatorname{Ir}(001)$ [63] and $\operatorname{Ir}(001)-\mathrm{Au}(1 \times 1)$ [64], spinfilter surfaces with high figure of merit and long lifetime in UHV have been found. For high- $Z$ materials, the diffraction process is highly spin selective, where usable maxima of the spin asymmetry function reach $80 \%$ [64]. The dimensionality of multichannel spin detection methods, spanning from the $1 D$ approach of the ToF instruments (Section 4.2) to the $3 D$ approach (see below) is sketched in Fig. 26. In this section we will critically examine the conventional $E-\theta$ acquisition mode as discussed in Section 3 , addressing the technical obstacles and possible solutions. This approach (panel (b)) is compared to the spin-filtered momentum microscope using a dispersive analyzer as developed at the MPI for Microstructure Physics in Halle (c). A challenging future prospect is the possibility of $3 D$ multichannel spin detection (d) via implementing an imaging spin filter into the ToF $k$-microscope discussed in Section 5.1.

Multichannel spin detection in combination with a conventional ARPES analyzer, pioneered by Kolbe et al. $[18,65,120]$, is still under development. From the electron-optical point of view, this is the most demanding case of use because the exit field of an ARPES analyzer operated in $E-\theta$ mode is very large (as large as $40 \times 20 \mathrm{~mm}^{2}$ for state-of-the-art hemispherical analyzers (HA)). Converting the electron beam from such a large field into a parallel beam on the surface of the spinfilter crystal (as schematically sketched in Figs. 7 and 8) is practically impossible, so that the existing prototypes use reduced fields of $20 \times 20 \mathrm{~mm}^{2}$ or less. Moreover, the beam profile in the exit field of a HA is strongly astigmatic, as the divergence in the angular-dispersing plane is much larger than in the energy-dispersive plane. Another serious obstacle is the chromatic aberration caused by the energy spread (typically $10 \%$ of the pass energy) across the exit field. There is potential for improvement, especially by optimizing the properties of the entrance lens of the HA. The lenses of the present generation of instruments are optimized for large source area and high resolution in the exit plane where the image detector $(\mathrm{MCP} /$ screen combination or delay-line detector) is normally located. Optimization during half a century has led to excellent energy and angle resolution of the order of $1 \mathrm{meV}$ and $0.1^{\circ}$ (for small angular ranges) [2,3]. However, the improvements came at the cost of considerable beam tilt and strong astigmatism in the exit plane. Both of these facts are less relevant for common spin-integral spectroscopy because the image detector is placed in the exit plane. However, they strongly hamper multichannel spin detection. Hence, an optimized instrument has to account for proper conditions at the entrance side of the HA. Two further factors, discussed in detail in [21], concern the surface Debye temperature, which is important since the spin detector should be operated at room temperature. Further, the mosaic spread of the spinfilter crystal sets a limit to the achievable point resolution. The surface Debye temperature of the $\operatorname{Ir}\left(\begin{array}{ll}0 & 01) \\ - & \mathrm{Au}\end{array}\right.$ system is surprisingly high and the mosaicity only becomes visible at a large number of imaged data points [21]. The limits set by the specific electron-optical properties are more serious at the present stage of development.

The first commercial instrument is shown in Fig. 27. It employs a magnetic spin rotator lens between analyzer exit and spinfilter for asymmetry reversal and for the selection of both transversal spin components. The spinfilter crystal is mounted on a 5-axes manipulator to precisely align for specular reflection along the center of the upper (vertical) lens system. The crystal can be retracted in order to give way for spin-integral measurements in the horizontal branch. Delay-line detectors at the end of both branches record single counting events with low background $\left(<1 \mathrm{cps} / \mathrm{cm}^{2}\right)$ and high dynamic range $\left(10^{6}\right)$

In the instrument shown in Fig. 27, asymmetry reversal is facilitated by a magnetic lens. However, there are many possibilities of asymmetry reversal and most of them have not yet been used or tested. The easiest cases are those for which the spin polarization of the electrons can be reversed, for example (i) by reversing the sample magnetization (as in $[18,65]$ ) or (ii) by reversing the helicity of circularly polarized light in optical spin orientation (see, 


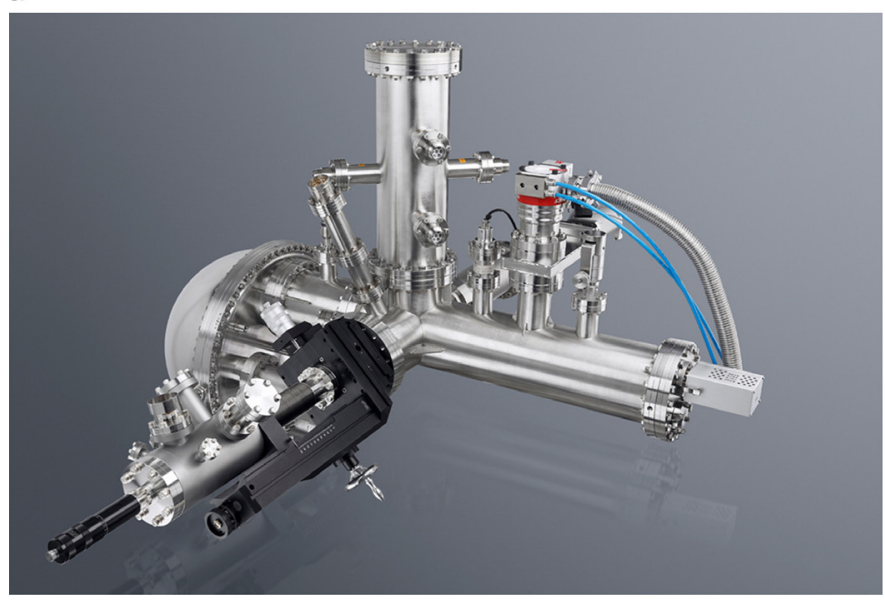

b

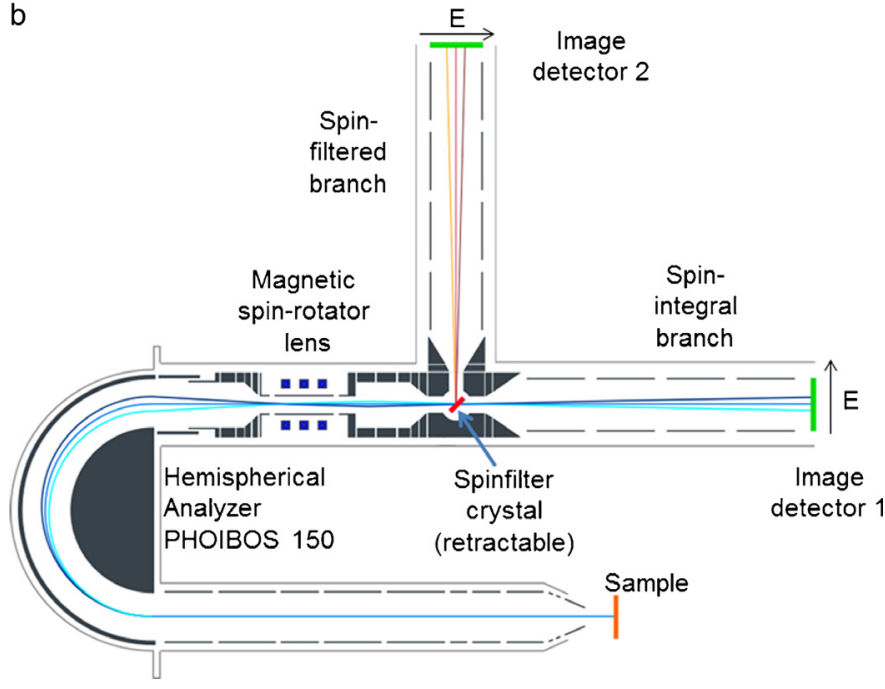

Fig. 27. First commercial multichannel spin polarimeter in conjunction with a hemispherical energy analyzer. This instrument comprises a magnetic spin rotator lens [67] for asymmetry reversal and for selection of both transversal spin components (figures courtesy SPECS GmbH).

e.g. [121]). In these cases, the spinfilter can be operated unipolar, like $\mathrm{W}(001)$ at $26 \mathrm{eV} / 45^{\circ}$, or clean or Au-passivated $\operatorname{Ir}(001)$ around $40 \mathrm{eV}$. However, for Rashba-type spin structures, the primary polarization cannot be reversed, so that asymmetry reversal must be done with the spinfilter in one of the following ways: (iii) by use of a bipolar working point like $\operatorname{Ir}(001)-\mathrm{Au}[21]$ at $10.25 / 11.5 \mathrm{eV}$ and $45^{\circ}$ (with a usable energy interval less than $1 \mathrm{eV}$ ) or W(0 01 ) at $22 / 26 \mathrm{eV}$ and $56^{\circ}$ (large usable interval of several eV, cf. Fig. 9(e)), (iv) spin rotation by a magnetic lens (see Fig. 27) or full 3D spin manipulation [122a], (v) rotation of the spinfilter-detector assembly about the axis of the incoming electron beam (a prototype is under construction for a spin-HAXPES experiment), (vi) exploitation of asymmetry change by azimuthal rotation of the spinfilter crystal about its surface normal * (also giving access to the longitudinal spin component [122b]), (vii) reversing the magnetization direction of an exchangetype spinfilter like a Co-film [25] or oxygen-passivated $\mathrm{Fe}(001)$ surface [68] or finally (viii) utilizing mirror symmetries in the spinfiltered pattern (analogously to the determination of dichroism asymmetries in [100]). This last possibility might be useful for the momentum microscope approaches, Fig. 26((c) and (d)).

The problems associated with the large exit field of the analyzer are strongly reduced at high energies, in the domain of Mott scattering. This possibility has been recently discussed by Strocov et al. [71]. An intriguing advantage of the instruments based on Mott scattering (for their recent realization see, for example, [123a,123b]) is their long-term stability, making it possible to work with such instruments for several years (as one of the authors did $[121,124])$, without maintenance. The future will show whether this advantage can compensate the rather low spin-orbit asymmetry of 0.22 and, in turn, the small single-channel figure of merit of Mott scattering. Detailed simulations indicate that Mott scattering seems to be an interesting alternative approach. The iMott design of Strocov et al. [71] uses imaging-type electron optics, accepting inherently divergent electron rays from the analyzer focal plane and those scattered at the Au target. The expected gain in efficiency is about $10^{4}$, the same order of magnitude as found for the $\mathrm{W}(001)$ based prototype by Kolbe et al. [18]. The efficiency-gain factor $G$ defined in [71] is equivalent to the definition of the multichannel figure of merit in $[18,20,21]$.

Spin-filtered momentum microscopy using a dispersive analyzer is discussed in the article by Suga and Tusche [23]. Here we compare the performance of the conventional $E-\theta$ mode (Section 3 ) with the spin-filtered $k$-microscope. The latter acquires a spin-filtered momentum image with a size exceeding the full Brillouin zone at the selected energy. An energy resolution of $12 \mathrm{meV}$ along with a $k$-resolution of $<5 \times 10^{-3} \AA^{-1}$ has been achieved. The low-energy working point of Au-passivated $\operatorname{Ir}(001)$ allows asymmetry reversal with the following parameters [21]: scattering energy $10.25 \mathrm{eV}(11.5 \mathrm{eV})$, reflectivity $1.3 \%$ (2.3\%), spin asymmetry $-65 \%(+57 \%)$, yielding a figure of merit of $5.5 \times 10^{-3}$ $\left(7.4 \times 10^{-3}\right)$. The high-energy working point around $40 \mathrm{eV}$ (with a usable energy interval of several $\mathrm{eV}$ ) has a polarization maximum of about $80 \%$ [64] and a figure of merit of $1.2 \times 10^{-3}$. The corresponding two-dimensional figure of merit at $10.25 \mathrm{eV}$ is given by $\mathrm{FoM}_{2 \mathrm{D}}=N \times \mathrm{FoM}_{\text {single }}=37$ (with $N=5000$ simultaneously acquired data points, corresponding to 80 resolved points along the image diagonal). $\mathrm{N}$ is the number of resolved $\left(k_{x}, k_{y}\right)$ data points; the resolution of the image detector is an order of magnitude higher.

According to the definition that the FoM is the statistically relevant quantity [69] and counting the resolvable channels, we can directly compare this result with the value of $\mathrm{FoM}_{2 \mathrm{D}}=1.7$ found for the setup of Kolbe et al. [18] in $E-\theta$ mode, cf. Section 3, with

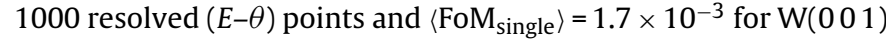
at the working point of $\theta=45^{\circ} / 26 \mathrm{eV}$ scattering energy. Extrapolating these figures to the higher FoM $\mathrm{M}_{\text {single }}$ of the Ir-Au spinfilter $\left(7.4 \times 10^{-3}\right)$ and a modern $\mathrm{HA}$ with a resolution in the $10 \mathrm{meV}$ range (yielding > 100 resolved energy points), we arrive at a similar figure of merit as reached by the MPI Halle system. In both cases, there is potential for improvement, as mentioned before. Simulations revealed that an energy resolution in the $1 \mathrm{meV}$ range can be reached with improved entrance optics.

Finally, we will briefly discuss the emerging technique of ToF momentum microscopy with imaging spin filter, simultaneously resolving $k$-space, time and spin. This approach is being developed by the University of Mainz group in cooperation with the MPI in Halle. The electron-optical column is identical to the momentum microscope described in [22,23], except that it implements a low-energy drift tube and a DLD for parallel detection of many energy slices via the time of flight. It adds the third dimension to multichannel spin-resolved data acquisition as illustrated in Fig. 26(d). The schematic layout of this instrument is shown in Fig. 28. The spin-filtered version of the ToF $k$-microscope comprises two identical ToF branches with two DLDs, one for taking spinintegral measurements in the straight-through direction and one under twice the scattering angle for spin-filtered measurements. The spinfilter crystal is retractable, so that fast change between spin-integral and spin-filtered detection is possible. The number of energy slices acquired depends on the energy resolution of the ToF unit in relation to the usable width of the energy interval. The latter is limited primarily by the chromatic aberration of the 


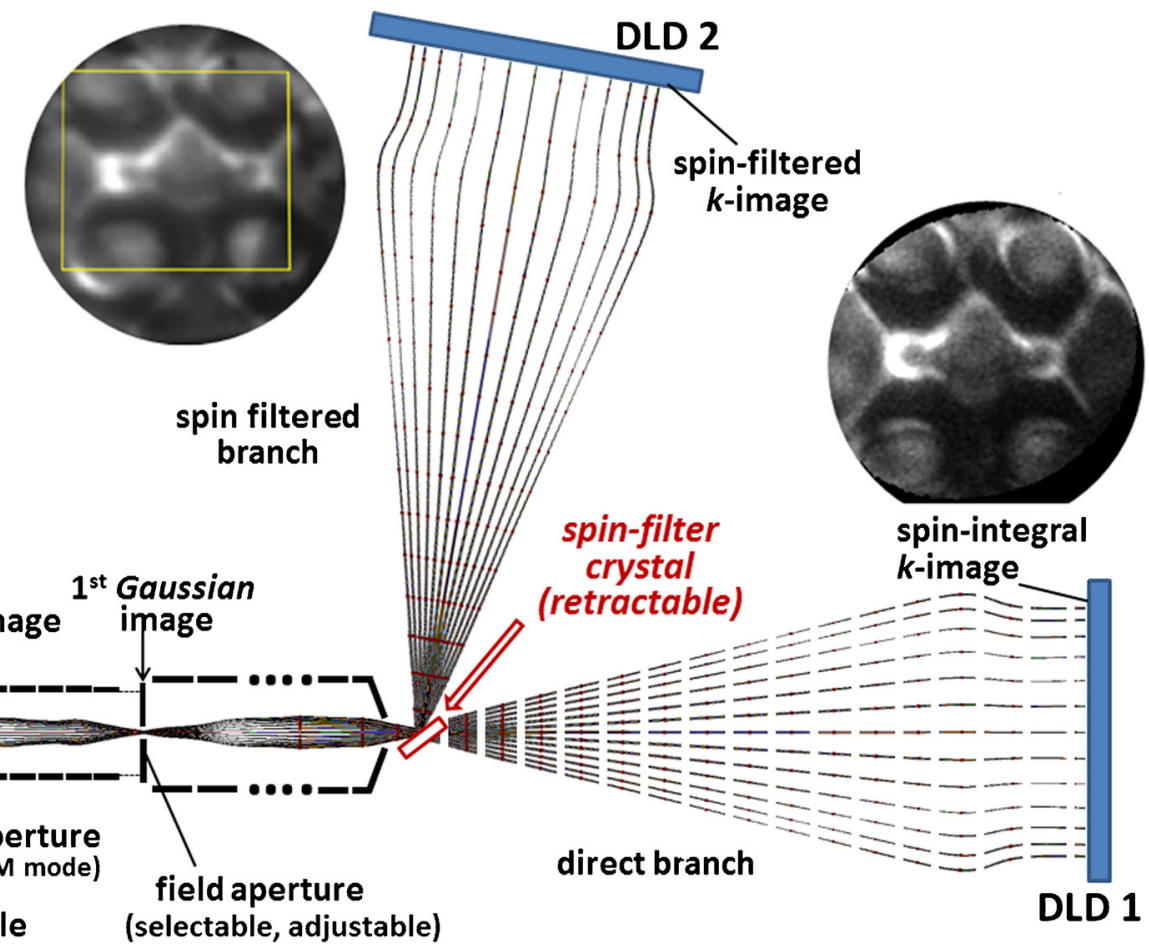

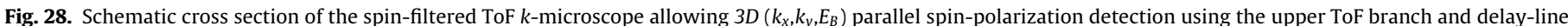

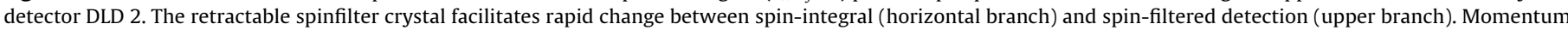

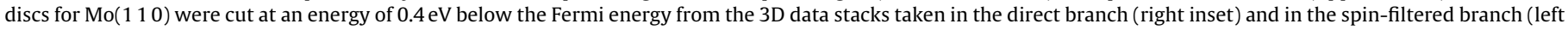
inset). Details of these surface bands are discussed in Section 5.2.

electron-optical system. In the experiments shown in Section 5.2 , an energy window of $1-2 \mathrm{eV}$ could be imaged with $\sim 10^{5}$ $\left(k_{x}, k_{y}, E_{B}\right)$ data voxels acquired simultaneously. The parallel energy acquisition enters into the multichannel figure of merit as $\mathrm{FoM}_{3 \mathrm{D}}=N_{E} \times N_{k} \times$ FoM $\mathrm{M}_{\text {single }}$, with $N_{E}$ and $N_{k}$ denoting the number of resolved energy slices and $k$-points. Although the first spin-filtered image sequences with good quality have been obtained (see lefthand inset of Fig. 28), it is not clear yet how many $\left(k_{x}, k_{y}, E_{B}\right)$ data voxels can be transferred by the spin filter.

For the ToF $k$-microscope, a further limitation on the usable energy interval can originate from the characteristics of the spin filter itself. As discussed in Section 3, the working point around $40 \mathrm{eV}$ for Ir and Ir-Au is characterized by a large usable energy interval of several $\mathrm{eV}$, imposing no further limit on the energy range. However, the bipolar asymmetry profile at the $10.25 / 11.5 \mathrm{eV}$ working point with high spinfilter performance [21] is rather narrow. This working point is thus particularly useful for ToF $k$-microscopy with ultimate energy resolution. In conclusion, this new approach has the potential for a very high multichannel FoM. It requires new strategies for dealing with the energy-dependence of the spin asymmetry and the chromatic aberration of the electronoptical system. In the future, the usable energy interval could be expanded by dynamic aberration correction as discussed in [125]. The method will prove its advantages in low-intensity experiments (like HAXPES), in time-resolved experiments, for reactive or radiation-sensitive (organic) samples and in high-resolution applications, where the number of resolved time slices can be very large.

\subsection{Advances in highly-parallel 3D electron detection}

Considering the development of high-brilliance light sources in the lab and at large-scale facilities, the electron detector itself may limit the overall performance of a high-throughput experiment. Here we will discuss the status and emerging achievements in highly-parallelized electron detection using the DLD principle. The preceding sections have shown that in future photoemission experiments $3 D\left(k_{x}, k_{y}, E_{B}\right)$ parallel data acquisition comes with striking advantages, e.g. in terms of the large number of simultaneously acquired voxels, the single-particle detection principle with a dynamic range of $10^{6}$ and its true real-time capability. The latter is particularly important in coincidence experiments [126]. Similar arguments hold for high-throughput ultrafast photon detection [127].

The DLD technique has improved substantially within the last few years. Time resolution (peak FWHM) is typically below 150 ps (time-slice resolution $13.7 \mathrm{ps}$ ) and the spatial resolution is below $80 \mu \mathrm{m}$ (pixel resolutions $12 \mu \mathrm{m}$ to $48 \mu \mathrm{m}$, depending on anode size). Maximum random count rates are 5 to $8 \mathrm{Mcps}$. In the near future, the time peak FWHM will improve towards 50 ps (peak reproducibility $5 \mathrm{ps}$ ). Lithographically fabricated delay-line meanders replaced the former wire-wrap technique, leading to an enhanced reproducibility for batch production and more flexibility in impedance matching.

The operation principle of DLDs is discussed in detail in [96,97]. A DLD requires a multichannelplate (MCP) intensifier, generating fast charge pulses of typically $10^{6}-10^{7}$ electrons. When crossing the delayline anode, each charge pulse induces an electrical pulse on two crossed meanders. The architecture of a DLD is based on the readout of time differences between the two ends of a delay line (yielding the position) and the time distance of the centers of gravity of the signals with respect to an external clock (trigger pulse of a laser or bunch marker of a synchrotron source) for the arrival time of the event. A pilot ToF-PEEM experiment was performed in cooperation with the group of H. Schmidt-Böcking (Univ. of Frankfurt) [96]. New anode developments have started at the Univ. of Mainz and have since been continued and commercialized by the spin-off Surface Concept [98]. The discrimination of more than two simultaneous events is practically impossible, 
(a)

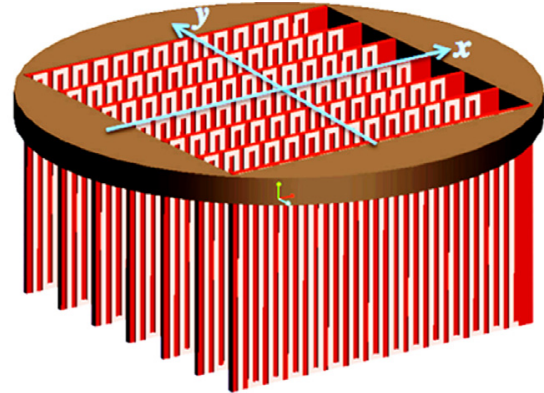

(b)

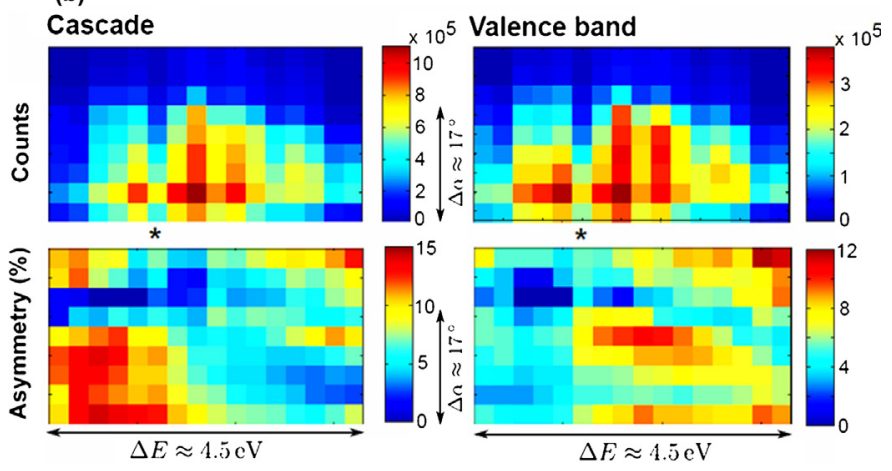

Fig. 29. (a) Schematic view of a multi-segment DLD with the delay-line meanders extending perpendicular to the image plane. (b) First results taken with the 16segment DLD prototype at the free electron laser FLASH at Desy, Hamburg, with the multichannel spin polarimeter shown in Fig. 8. Intensity (upper row) and spin asymmetry (lower row) are shown for the region of the secondary-electron cascade (left) and the valence band region (right). The hemispherical analyzer was run in the $E-\theta$ mode with simultaneous acquisition of energy and angle intervals of $4.5 \mathrm{eV}$ and $17^{\circ}$, respectively (from [130]).

which is also the case for the hexanode [128] or similar designs. The straightforward solution to this problem is parallelization of many detector segments [129]. DLD multi-anode designs have reached the stage of series production for double or quad layouts (with burst rates above $100 \mathrm{Mcps}$ and permanent randomly measured 40 Mcps). Recently, a 16-segment solution was developed and used in an experiment at the free-electron laser FLASH [130]. Striving for even higher integration, a 128-segment DLD is under development for the SQS photon spectrometer at the European XFEL [127] and a 256-segment solution will be used by CFEL, Hamburg.

The architecture of a multi-segment DLD (stacked separated $1 D$ delay lines) makes use of the third dimension as shown in Fig. 29(a). The meanders extend perpendicular to the image plane, unlike in the standard DLD [96]. The edges of the meanders serve as 1dimensional antennas; the spatial resolution along one meander is given by the precision of the center of gravity determination of the induced electric pulse groups to the ends of each individual delayline segment. The resolution in x-direction can be many hundred pixels as in regular DLDs, while in y-direction it is given by the number of $1 D$ delay line segments, presently up to 256 .

For the 256-segment DLD, the multi-hit capability will exceed 100 events per pulse, with each $1 D$ delay line being capable to resolve double hits. The maximum permanent random count rate will be up to 100 Mcps, being limited by the physics of the MCPs and, partly, by the realistic long term average data transfer speed. Carrying its individual time coordinate on a 150 ps time scale, each event can be correlated with the corresponding microbunch of the bunch train of an FEL. The limits for high countrate operation are set by the limitations of the available MCPs. A single MCP pore needs at least a few $100 \mu$ s to recharge. When the events cluster in space and/or time, the count-rate limits will be lower, depending on the density of MCP pores in the exposed areas. A DLD anode operation requires a minimum MCP amplification of about $10^{6}$. Partially discharged pores may deliver insufficient charge in pulses at high local hit rates. For extremely high intensities, the lifetime of MCPs may be an issue: roughly, a single pore of the back MCP in a DLD design can fire a maximum of $10^{14}$ electrons in its lifetime. This translates to only $10^{8}$ incoming electrons per pore in total at an amplification of $10^{6}$. The readout system has a few bottlenecks as well: the local memory capacity of the current readout design may be limited to store a few 100 million hit event data sets before transfer. The data stream can go to the PC by Ethernet, PCle or USB3.0 (giving a long-time average transfer data rate of about 60-80 million hit event data sets per second when 4 bytes are transferred per hit).

Fig. 29(b) shows the result of a measurement taken with the 16-segment DLD prototype at the free electron laser FLASH in Hamburg. FLASH delivers bunch trains of up to 800 microbunches (in the present experiment 350 ) at $1 \mu$ s spacing at a repetition rate of $10 \mathrm{~Hz}$, for a total of only 3500 photon pulses per second. Such conditions call for parallelization on the detector side. This detector can accept up to about 60 multihits per microbunch that are all registered as individual events including their time coordinates, thus assigning each event to a certain microbunch [130]. This is crucial for time-dependent experiments because the microbunches come with a significant jitter due to the SASE principle [131]. Each event is labelled by its individual pump-probe delay, because, in parallel, the "time flag" of each individual microbunch is recorded. The data of Fig. 29(b) were recorded behind the imaging spin filter. Intensity as well as spin asymmetry are shown for the region of the secondary cascade electrons (left column) and for the valence range (right column). Despite the poor statistics, this pilot study demonstrated that multi-hit spin analysis is possible in principle. However, due to the small number of photon pulses and due to severe space-charge problems [88] the noise level in the data is very large.

\section{Note added in proof}

Recently it was shown for the $\operatorname{Ir}(001)$ spinfilter that azimuthal rotation by $30^{\circ}$ leads to a reversal of the spin asymmetry, being the most elegant way of asymmetry "switching” (E. Schäfer et al., to be published).

\section{Acknowledgements}

The authors would like to thank all people being involved in the developments discussed in this paper, in particular to the coworkers and colleagues S. Chernov, D. Kutnyakhov, R. Wallauer, F. Schertz, D. Panzer, and S. A. Nepijko. Very deep thanks go to our dear colleagues in Halle, Prof. J. Kirschner and his coworkers C. Tusche and A. Krasyuk, without whom the developments in Mainz would not have been possible. Further thanks are due to A. Oelsner (Surface Concept $\mathrm{GmbH}$ ) for supplying info about the delay-line detector, to O. Schaff (SPECS GmbH) for providing info about the first commercial multichannel spin detector and to B. Schönhense (Imperial College London) for a critical reading of the manuscript. For fruitful cooperation we thank the staff of BESSY (in particular C. Schüssler and A. Föhlisch), R. Claessen and M. Scholz (Univ. Würzburg) for providing the $\mathrm{Bi}_{2} \mathrm{Te}_{3}$ crystal as well as Y. Acremann (ETH Zurich) and W. Wurth (CFEL at DESY, Hamburg) for cooperation at FLASH. Financial support by DFG (Scho 341/9 and EL 172/18-1), BMBF (05K12UM2, 05K12EF1, 05K13UM1, 05K13UM2 and 05K13UM4), Stiftung Innovation (project 1038), the Center for Innovative and Emerging Materials CINEMA and the Graduate School of Excellence MAINZ is gratefully acknowledged. 


\section{References}

[1] K. Siegbahn, C. Nordling, A. Fahlman, R. Nordberg, K. Hamrin, J. Hedman, G. Johansson, T. Bergmark, S.-E. Karlsson, I. Lindgren, B. Lindberg, ESCA, Atomic, Molecular and Solid State Structure Studied by Means of Electron Spectroscopy, Almqvist and Wiksells, Uppsala, Sweden, 1967.

[2] N. Mårtensson, P. Baltzer, P.A. Bruhwiler, J.-O. Forsell, A. Nilsson, A. Stenborg, B. Wannberg, J. Electron. Spectrosc. Relat. Phenom. 70 (1994) 117.

[3] V.N. Strocov, M. Shi, M. Kobayashi, C. Monney, X. Wang, J. Krempasky, T. Schmitt, L. Patthey, H. Berger, P. Blaha, Phys. Rev. Lett. 109 (2012) 086401.

[4] F. Krausz, M. Ivanov, Rev. Mod. Phys. 81 (2009) 163.

[5] A. Rundquist, C.G. Durfee III, Z. Chang, C. Herne, S. Backus, M.M. Murnane, H.C. Kapteyn, Science 280 (1998) 5368.

[6] B.W.J. McNeil, N.R. Thompson, Nat. Photonics 4 (2010) 814

[7] E.I. Rashba, Fiz. Tver. Tela (Leningrad) 2 (1960) 1224; Y.A. Bychkov, E.I. Rashba, JETP Lett. 39 (1984) 78.

[8] M.Z. Hasan, J.E. Moore, Annu. Rev. Condens. Matter Phys. 2 (2011) 55.

[9] G. Schönhense, A. Oelsner, A. Krasyuk, C.M. Schneider, M. Bauer, M. Aeschlimann, Recent Trends in Charged Particle Optics, Czechoslovak Microscopy Society, Brno, 2002, 80-238-8986-9, 90.

[10] S.-B. Choe, Y. Acremann, A. Scholl, A. Bauer, A. Doran, J. Stöhr, H.A. Padmore, Science 304 (2003) 420.

[11] J. Vogel, W. Kuch, M. Bonfim, J. Camarero, Y. Pennec, F. Offi, K. Fukumoto, J. Kirschner, A. Fontaine, S. Pizzini, Appl. Phys. Lett. 82 (2003) 2299.

[12] A. Krasyuk, A. Oelsner, S.A. Nepijko, A. Kuksov, C.M. Schneider, G. Schönhense, Appl. Phys. A 76 (2003) 863.

[13] O. Schmidt, M. Bauer, C. Wiemann, R. Porath, M. Scharte, O. Andreyev, G. Schönhense, M. Aeschlimann, Appl. Phys. B 74 (3) (2002) 223

[14] M. Cinchetti, A. Gloskovskii, S.A. Nepjiko, G. Schönhense, H. Rochholz, M. Kreiter, Phys. Rev. Lett. 95 (2005) 047601.

[15] L. Chelaru, F.-J. Meyer zu Heringdorf, Surf. Sci. 601 (2007) 4541.

[16] A. Kubo, N. Pontius, H. Petek, Nano Lett. 7 (2007) 470.

[17] M. Bauer, C. Wiemann, J. Lange, D. Bayer, M. Rohmer, M. Aeschlimann, Appl. Phys. A 88 (3) (2007) 473.

[18] M. Kolbe, P. Lushchyk, B. Petereit, H.J. Elmers, G. Schönhense, A. Oelsner, C. Tusche, J. Kirschner, Phys. Rev. Lett. 107 (2011) 207601

[19] O. Schaff (SPECS GmbH), private communication.

[20] C. Tusche, M. Ellguth, A.A. Uenal, C.-T. Chiang, A. Winkelmann, A. Krasyuk, M. Hahn, G. Schönhense, J. Kirschner, Appl. Phys. Lett. 99 (2011) 032505.

[21] D. Vasilyev, C. Tusche, F. Giebels, H. Gollisch, R. Feder, J. Kirschner, J. Electron. Spectrosc. Relat. Phenom. 199 (2015) 10.

[22] C. Tusche, A. Krasyuk, J. Kirschner, Ultramicroscopy (2015), 0.1016/j.ultramic. 2015.03.020. (in print).

[23] S. Suga, C. Tusche, Volume 200.

[24] N. Müller, R. David, G. Snell, R. Kuntze, M. Drescher, N. Böwering, P. Stoppmanns, S.-W. Yu, U. Heinzmann, J. Viefhaus, U. Hergenhahn, U. Becker, J. Electron. Spectrosc. Relat. Phenom. 72 (1995) 187.

[25] K. Gotlieb, Z. Hussain, A. Bostwick, A. Lanzara, C. Jozwiak, Rev. Sci. Instrum. 84 (2013) 093904.

[26] M. Cinchetti, M. Sánchez Albaneda, D. Hoffmann, T. Roth, J.-P. Wuestenberg, M. Krauß, O. Andreyev, C.H. Schneider, M. Bauer, M. Aeschlimann, Phys. Rev. Lett. 97 (2006) 177201.

[27] A. Fognini, T.U. Michlmayr, G. Salvatella, C. Wetli, U. Ramsperger, T. Bahler, F. Sorgenfrei, M. Beye, A. Eschenlohr, N. Pontius, C. Stamm, F. Hieke, M. Dell'Angela, S. de Jong, R. Kukreja, N. Gerasimova, V. Rybnikov, A. AlShemmary, H. Redlin, J. Raabe, A. Föhlisch, H.A. Dürr, W. Wurth, D. Pescia, A. Vaterlaus, Y. Acremann, Appl. Phys. Lett. 104 (2014) 032402.

[28] G. Schönhense, A. Oelsner, O. Schmidt, G.H. Fecher, V. Mergel, O. Jagutzki, H. Schmidt-Böcking, Surf. Sci. 480 (2001) 180.

[29] G. Öhrwall, P. Karlsson, M. Wirde, M. Lundqvist, P. Andersson, D. Ceolin, B. Wannberg, T. Kachel, H. Dürr, W. Eberhardt, S. Svensson, J. Electron. Spectrosc. Relat. Phenom. 183 (2011) 125.

[30] P. Dziawa, B.J. Kowalski, K. Dybko, R. Buczko, A. Szczerbakow, M. Szot, E. Łusakowska, T. Balasubramanian, B.M. Wojek, M.H. Berntsen, O. Tjernberg, T. Story, Nat. Mater. 11 (2012) 1023.

[31] S. Eich, A. Stange, A.V. Carr, J. Urbancic, T. Popmintchev, M. Wiesenmayer, K. Jansen, A. Ruffing, S. Jakobs, T. Rohwer, S. Hellman, C. Chen, P. Matyba, L. Kipp, K. Rossnagel, M. Bauer, M.M. Murnane, H.C. Kapteyn, S. Mathias, M. Aeschlimann, J. Electron. Spectrosc. Relat. Phenom. 195 (2014) 231.

[32] K. Perzlmaier, M. Buess, C.H. Back, V.E. Demidov, B. Hillebrands, S.O. Demokritov, Phys. Rev. Lett. 94 (2005) 057202.

[33] A. Krasyuk, A. Oelsner, S.A. Nepijko, N.N. Sedov, A. Kuksov, C.M. Schneider, G. Schönhense, Appl. Phys. A 79 (2004) 1925.

[34] A. Krasyuk, F. Wegelin, S.A. Nepijko, H.J. Elmers, G. Schönhense, M. Bolte, C.M. Schneider, Phys. Rev. Lett. 95 (2005) 207201.

[35] J. Raabe, C. Quitmann, C.H. Back, F. Nolting, S. Johnson, C. Buehler, Phys. Rev. Lett. 94 (2005) 217204.

[36] C.M. Schneider, A. Kuksov, A. Krasyuk, A. Oelsner, D. Neeb, S.A. Nepijko, G. Schönhense, J. Morais, C. de Nadai, N.B. Brookes, Appl. Phys. Lett. 85 (2004) 2562.

[37] H. Stoll, A. Puzic, B. van Waeyenberge, P. Fischer, J. Raabe, M. Buess, T. Haug, R. Höllinger, C. Back, D. Weiss, G. Denbeaux, Appl. Phys. Lett. 84 (2004) 3328.

[38] B. van Waeyenberge, A. Puzic, H. Stoll, K.W. Chou, T. Tyliszczak, R. Hertel, M. Fähnle, H. Brückl, K. Rott, G. Reiss, I. Neudecker, D. Weiss, C.H. Back, G. Schütz, Nature 444 (2006) 461.
[39] A. Neudert, Y.W. Lai, R. Schäfer, M. Kustov, L. Schultz, J. McCord, Adv. Eng. Mater. 14 (2012) 601.

[40] J.P. Park, P. Eames, D.M. Engebretson, J. Berezovsky, P.A. Crowell, Phys. Rev. B: Condens. Matter 67 (2003) 020403.

[41] J. Levy, V. Nikitin, J.M. Kikkawa, A. Cohen, N. Samarth, R. Garcia, D.D. Awschalom, Phys. Rev. Lett. 76 (1996) 1948.

[42] S.A. Nepijko, A. Oelsner, A. Krasyuk, A. Gloskovskii, N.N. Sedov, C.M. Schneider, G. Schönhense, Appl. Phys. A 78 (2004) 47.

[43] K. Hild, J. Maul, G. Schönhense, H.J. Elmers, M. Amft, P.M. Oppeneer, Phys. Rev. Lett. 102 (2009) 057207.

[44] G.K.L. Marx, H.J. Elmers, G. Schönhense, Phys. Rev. Lett. 84 (2000) 5888.

[45] F. Wegelin, A. Krasyuk, D.A. Valdaitsev, S.A. Nepijko, H.J. Elmers, G. Schönhense, C.M. Schneider, Phys. Stat. Sol. B: Basic Solid State Phys. 246 (2009) 1476.

[46] D.A. Valdaitsev, A. Kukunin, J. Prokop, H.J. Elmers, G. Schönhense, Appl. Phys. A 80 (2005) 731.

[47] A. Hubert, R. Schäfer, Magnetic Domains, Springer, Berlin, 1998.

[48] H. Yu, R. Huber, T. Schwarze, F. Brandl, T. Rapp, P. Berberich, G. Duerr, D. Grundler, Appl. Phys. Lett. 100 (2012) 262412.

[49] C. Wiemann, D. Bayer, M. Rohmer, M. Aeschlimann, M. Bauer, Surf. Sci. 601 (2007) 4714

[50] F. Schertz, M. Schmelzeisen, R. Mohammadi, M. Kreiter, H.-J. Elmers, G. Schönhense, Nano Lett. 12 (2012) 1885.

[51] F. Schertz, M. Schmelzeisen, M. Kreiter, H.-J. Elmers, G. Schönhense, Phys. Rev. Lett. 108 (2012) 237602.

[52] P. Biagioni, J.S. Huang, L. Duo, M. Finazzi, B. Hecht, Phys. Rev. Lett. 102 (2009) 256801.

[53] S.W. Hell, Science 316 (2007) 1153.

[54] L. Novotny, B. Hecht, Principles of Nano-Optics, Cambridge Univ. Press, Cambridge, 2006.

[55] T. Guenther, C. Lienau, T. Elsaesser, M. Glanemann, V.M. Axt, T. Kuhn, S. Eshlaghi, A.D. Wieck, Phys. Rev. Lett. 89 (2002) 057401.

[56] M. Aeschlimann, T. Brixner, A. Fischer, C. Kramer, P. Melchior, W. Pfeiffer, C. Schneider, C. Strüber, P. Tuchscherer, D. Voronine, Science 333 (2011) 1723.

[57] P. Kahl, S. Wall, C. Witt, C. Schneider, D. Bayer, A. Fischer, P. Melchior, M. Hornvon Hoegen, M. Aeschlimann, F.-J. Meyer zu Heringdorf, Plasmonics 9 (2014) 1401.

[58] P. Klaer, G. Razinskas, M. Lehr, K. Krewer, F. Schertz, X.-F. Wu, B. Hecht, G. Schönhense, H.-J. Elmers, submitted.

[59] C. Lemke, C. Schneider, T. Leißner, D. Bayer, J.W. Radke, A. Fischer, P. Melchior, A.B. Evlyukhin, B.N. Chichkov, C. Reinhardt, M. Bauer, M. Aeschlimann, Nano Lett. 13 (3) (2013) 1053.

[60] T. Schmidt, S. Heun, J. Slezak, J. Diaz, K.C. Prince, G. Lilienkamp, E. Bauer, Surf. Rev. Lett. 5 (1998) 1287.

[61] F. Meier, H. Dil, J. Lobo-Checa, L. Patthey, J. Osterwalder, Phys. Rev. B: Condens. Matter 77 (2008) 165431

[62] T. Okuda, K. Miyamoto, A. Kimura, H. Namatame, M. Taniguchi, J. Electron. Spectrosc. Relat. Phenom. 201 (2015) 23.

[63] D. Kutnyakhov, P. Lushchyk, A. Fognini, D. Perriard, M. Kolbe, K. Medjanik, E. Fedchenko, S.A. Nepijko, H.J. Elmers, G. Salvatella, C. Stieger, R. Gort, T. Bähler, T. Michlmayer, Y. Acremann, A. Vaterlaus, F. Giebels, H. Gollisch, R. Feder, C. Tusche, A. Krasyuk, J. Kirschner, G. Schönhense, Ultramicrosc 130 (2013) 63.

[64] J. Kirschner, F. Giebels, H. Gollisch, R. Feder, Phys. Rev. B: Condens. Matter 88 (2013) 125419.

[65] M. Jourdan, J. Minar, J. Braun, A. Kronenberg, S. Chadov, B. Balke, A. Gloskovskii, M. Kolbe, H.J. Elmers, G. Schönhense, H. Ebert, C. Felser, M. Kläui, Nat. Commun. 5 (2014) 3974.

[66] M. Hahn, G. Schönhense, E. Arbelo Jorge, M. Jourdan, Appl. Phys. Lett. 98 (2011) 232503.

[67] O.Schaff (SPECS GmbH), Private communication; Patent no. 102010052088.8 and PCT/EP2011/070226.

[68] K. Miyamoto, A. Kimura, T. Okuda, M. Donath, J. Electron. Spectrosc. Relat. Phenom. 201 (2015) 53 (Available online 24 October 2014).

[69] J. Kessler, Polarized Electrons, Springer Tracts in Mod. Phys., Berlin, Heidelberg, 1985.

[70] C. Tusche, M. Ellguth, A. Krasyuk, A. Winkelmann, D. Kutnyakhov, P. Lushchyk, K. Medjanik, G. Schönhense, J. Kirschner, Ultramicroscopy 130 (2013) 70

[71] V. Strocov, V.N. Petrov, J.H. Dil, J. Synchrotron Radiat. 22 (2015) 708.

[72] D. Kutnyakhov, H.J. Elmers, G. Schönhense, C. Tusche, S. Borek, J. Braun, J. Minár, H. Ebert, Phys. Rev. B: Condens. Matter 91 (2015) 014416.

[73] (a) M. Kolbe, S. Chadov, E. Arbelo Jorge, G. Schönhense, C. Felser, H.-J. Elmers, M. Kläui, M. Jourdan, Phys. Rev. B: Condens. Matter 86 (2012) 024422; (b) J. Braun, M. Jourdan, A. Kronenberg, S. Chadov, B. Balke, M. Kolbe, A. Gloskovskii, H.J. Elmers, G. Schönhense, C. Felser, M. Kläui, H. Ebert, J. Minár، Phys. Rev. B: Condens. Matter 91 (2015) 195128.

[74] G. Müller, G. Eilers, Z. Wang, M. Scherff, R. Ji, K. Nielsch, C. Ross, M. Münzenberg, New J. Phys. 10 (2008) 123004.

[75] B. Koopmans, F. Dalla Longa, G. Malinowski, D. Steiauf, M. Fähnle, T. Roth, M. Cinchetti, M. Aeschlimann, Nat. Mater. 9 (2010) 259-265.

[76] E. Beaurepaire, J.C. Merle, A. Daunois, J.Y. Bigot, Phys. Rev. Lett. 76 (1996) 4250.

[77] C. Stamm, T. Kachel, N. Pontius, R. Mitzner, T. Quast, K. Holldack, S. Khan1, C. Lupulescu, E.F. Aziz, M. Wietstruk, H.A. Dürr, W. Eberhardt, Nat. Mater. 6 (2007) 740 .

[78] B. Koopmans, J.J.M. Ruigrok, F. Dalla Longa, W.J.M. de Jonge, Phys. Rev. Lett. 95 (2005) 267207. 
[79] A. Eschenlohr, M. Battiato, P. Maldonado, N. Pontius, T. Kachel, K. Holldack, R Mitzner, A. Föhlisch, P.M. Oppeneer, C. Stamm, Nat. Mater. 12 (2013) 332

[80] E. Turgut, D. La-o-vorakiat, J.M. Shaw, P. Grychtol, H.T. Nembach, D. Rudolf, R. Adam, M. Aeschlimann, C.M. Schneider, T.J. Silva, M.M. Murnane, H.C. Kapteyn, S. Mathias, Phys. Rev. Lett. 110 (2013) 197201.

[81] M. Battiato, K. Carva, P.M. Oppeneer, Phys. Rev. Lett. 105 (2010) 027203.

[82] B.Y. Mueller, B. Rethfeld, Phys. Rev. B: Condens. Matter 90 (2014) 144420.

[83] M. Plihal, D.L. Mills, Phys. Rev. B: Condens. Matter 58 (1998) 14407.

[84] A. Weber, F. Pressacco, S. Günther, E. Mancini, P.M. Oppeneer, C.H. Back, Phys. Rev. B: Condens. Matter 84 (2011) 132412.

[85] C. La-O-Vorakiat, M. Siemens, M.M. Murnane, H.C. Kapteyn, S. Mathias, M. Aeschlimann, P. Grychtol, R. Adam, C.M. Schneider, J.M. Shaw, H. Nembach, T.J. Silva, Phys. Rev. Lett. 103 (2009) 257402.

[86] S. Mathias, C. La-O-Vorakiat, P. Grychtol, P. Granitzka, E. Turgut, J.M. Shaw, R. Adam, H.T. Nembach, M.E. Siemens, S. Eich, C.M. Schneider, T.J. Silva, M. Aeschlimann, M.M. Murnane, H.C. Kapteyn, Proc. Natl. Acad. Sci. U.S.A. 109 (2012) 4792.

[87] M. Martins, M. Wellhoefer, J.T. Hoeft, W. Wurth, J. Feldhaus, R. Follath, Rev. Sci. Instrum. 77 (2006) 115108.

[88] A. Fognini, G. Salvatella, T.U. Michlmayr, C. Wetli, U. Ramsperger, T. Bähler, F. Sorgenfrei, M. Beye, A. Eschenlohr, N. Pontius, C. Stamm, F. Hieke, M. Dell'Angela, S. de Jong, R. Kukreja, N. Gerasimova, V. Rybnikov, H. Redlin, J. Raabe, A. Föhlisch, H.A. Dürr, W. Wurth, D. Pescia, A. Vaterlaus, Y. Acremann, New J. Phys. 16 (2014) 043031.

[89] G. Snell, M. Drescher, N. Müller, U. Heinzmann, U. Hergenhahn, J. Viefhaus, F Heiser, U. Becker, N. Brookes, Phys. Rev. Lett. 76 (1996) 3923; G. Snell, B. Langer, M. Drescher, N. Müller, B. Zimmermann, U. Hergenhahn, J. Viefhaus, U. Heinzmann, U. Becker, Phys. Rev. Lett. 82 (1999) 2480

[90] C. Jozwiak, J. Graf, G. Lebedev, N. Andresen, A.K. Schmid, A.V. Fedorov, F. El Gabaly, W. Wan, A. Lanzara, Z. Hussain, Rev. Sci. Instrum. 84 (2010) 053904.

[91] F.U. Hillebrecht, R.M. Jungblut, L. Wiebusch, Ch. Roth, H.B. Rose, D. Knabben, C. Bethke, N.B. Weber, St. Manderla, U. Rosowski, E. Kisker, Rev. Sci. Instrum. 73 (2002) 1229

[92] C. Jozwiak, C.-H. Park, K. Gotlieb, C. Hwang, D.-H. Lee, S.G. Louie, J.D. Denlinger, C.R. Rotundu, R.J. Birgeneau, Z. Hussain, A. Lanzara, Nat. Phys. 9 (2013) 293.

[93] E. Bauer, Ultramicroscopy 17 (1985) 51; E. Bauer, Rep. Prog. Phys. 57 (1994) 895

[94] A. Winkelmann, M. Ellguth, C. Tusche, A.A. Ünal, J. Henk, J. Kirschner, Phys. Rev. B: Condens. Matter 86 (2012) 085427.

[95] A. Winkelmann, A.A. Ünal, C. Tusche, M. Ellguth, C.-T. Chiang, J. Kirschner, N.J. Phys. 14 (2012) 083027

[96] A. Oelsner, O. Schmidt, M. Schicketanz, M. Klais, G. Schönhense, V. Mergel, O. Jagutzki, H. Schmidt-Böcking, Rev. Sci. Instrum. 72 (2001) 3968.

[97] A. Oelsner, M. Rohmer, C. Schneider, Daniela Bayer, G.M. Schönhense, M. Aeschlimann, J. Electron. Spectrosc. Relat. Phenom. 178-179 (2010) 317.

[98] 〈http://www. surface-concept.com〉.

[99] H. Spiecker, O. Schmidt, Ch. Ziethen, D. Menke, U. Kleineberg, R.C. Ahuja, M. Merkel, U. Heinzmann, G. Schönhense, Nucl. Instrum. Methods Phys. Res., Sect. A: Accel. Spectrom. Detect. Assoc. Equip. 406 (1998) 499.

[100] S. V. Chernov, K. Medjanik, C. Tusche, D. Kutnyakhov, S.A. Nepijko, A. Oelsner, J. Braun, J. Minár, S. Borek, H. Ebert, H.J. Elmers, J. Kirschner, and G. Schönhense, under review.

[101] E. Rotenberg, J.W. Chung, S.D. Kevan, Phys. Rev. Lett. 82 (1999) 4066

[102] K. Miyamoto, A. Kimura, K. Kuroda, T. Okuda, K. Shimada, H. Namatame, M. Taniguchi, M. Donath, Phys. Rev. Lett. 108 (2012) 066808.

[103] K. Miyamoto, A. Kimura, T. Okuda, K. Shimada, H. Iwasawa, H. Hayashi, H. Namatame, M. Taniguchi, M. Donath, Phys. Rev. B: Condens. Matter 86 (2012) 161411.

[104] J. Braun, K. Miyamoto, A. Kimura, T. Okuda, M. Donath, H. Ebert, J. Minár, New J. Phys (2014) 015005
[105] 〈https://www.helmholtz-berlin.de (14.11.2014)

[106] J. Hölzl, F.K. Schulte, Work Function of Metals, vol. 85, Springer Tracts in Modern Physics, Berlin, 1979.

[107] G. Schönhense, Phys. Scr. T31 (1990) 255.

[108] N.A. Cherepkov, G. Schönhense, Europhys. Lett. 24 (1993) 79.

[109] G.H. Fecher, Europhys. Lett. 29 (1995) 605.

[110] H.-J. Noh, H. Koh, S.-J. Oh, J.-H. Park, H.-D. Kim, J.D. Rameau, T. Valla, T.E. Kidd, P.D. Johnson, Y. Hu, Q. Li, EPL 81 (2008) 57006.

[111] Y.L. Chen, J.G. Analytis, J.-H. Chu, Z.K. Liu, S.-K. Mo, X.L. Qi, H.J. Zhang, D.H Lu, X. Dai, Z. Fang, S.C. Zhang, I.R. Fisher, Z. Hussain, Z.-X. Shen, Science 325 (2009) 178

[112] S. Hellmann, T. Rohwer, M. Kalläne, K. Hanff, C. Sohrt, A. Stange, A. Carr, M.M Murnane, H.C. Kapteyn, L. Kipp, M. Bauer, K. Rossnagel, Nat. Commun. 3 (2012) 1069.

[113] C. Sohrt, A. Stange, M. Bauer, K. Rossnagel, Faraday Discuss 171 (2014) 243.

[114] T. Rohwer, S. Hellmann, M. Wiesenmayer, C. Sohrt, A. Stange, B. Slomski, A Carr, Y. Liu, L.M. Avila, M. Kallaene, S. Mathias, L. Kipp, K. Rossnagel, M. Bauer Nature 471 (2011) 490

[115] A.X. Gray, C. Papp, S. Ueda, B. Balke, Y. Yamashita, L. Plucinski, J. Minár, J Braun, E.R. Ylvisaker, C.M. Schneider, W.E. Pickett, H. Ebert, K. Kobayashi, C.S Fadley, Nat. Mater. 10 (2011) 759

[116] G. Schönhense, K. Medjanik, C. Tusche, M. de Loos, B. van der Geer, M. Scholz, F. Hieke, N. Gerken, J. Kirschner, W. Wurth, Ultramicroscopy (2015), http:// dx.doi.org/10.1016/j.ultramic.2015.05.015

[117] M. Dell'Angela, T. Anniyev, M. Beye, R. Coffee, A. Föhlisch, J. Gladh, S. Kaya, T. Katayama, O. Krupin, A. Nilsson, D. Nordlund, W.F. Schlotter, J.A. Sellberg, F. Sorgenfrei, J.J. Turner, H. Ostrom, H. Ogasawara, M. Wolf, W. Wurth, Struc Dyn. 2 (2015) 025101.

[118] S.B. van der Geer, O.J. Luiten, M.J. de Loos, G. Pöplau, U. van Rienen, Inst. Phys Conf. Sci. 175 (2005) 101, 〈http://ww.pulsar.nl/gpt〉.

[119] G. Pöplau, U. van Rienen, S.B. van der Geer, M.J. de Loos, IEEE Trans. Magn. 40 (2004) 714.

[120] German Patent DE 102005045622 B4

[121] G. Schönhense, Appl. Phys. A 41 (1986) 39.

[122] (a) T. Duden, E. Bauer, Rev. Sci. Instrum. 66 (1995) 2861;

(b) P. Bauer, R. Feder, N. Müller, Solid State Commun. 36 (1980) 251.

[123] (a) V.N. Petrov, M. Landolt, M.S. Galaktionov, B.V.Yushenkov, Rev. Sci. Instrum. 68 (1997) 4385;

(b) M. Hoesch, T. Greber, V.N. Petrov, M. Muntwiler, M. Hengsberger, W. Auwaerter, J. Osterwalder, J. Electron. Spectrosc. Relat. Phenom. 124 (2002) 263.

[124] G. Schönhense, Phys. Rev. Lett. 44 (1980) 640.

[125] G. Schönhense and H. Spiecker, J. Vac. Sci. Technol. B 20 (2002) 2526; US Pat $6,737,647 \mathrm{~B} 2$

[126] M. Huth, C.-T. Chiang, A. Trützschler, F.O. Schumann, J. Kirschner, W. Widdra, Appl. Phys. Lett. 104 (2014) 061602;

C.-T. Chiang, M. Huth, A. Trützschler, M. Kiel, F.O. Schumann, J. Kirschner, W. Widdra, New J. Phys. 17 (2015) 013035

[127] M. Turcato, P. Gessler, S. Hauf, M. Kuster, M. Meyer, J. Nordgren, J. SztukDambietz, C. Youngman, J. Instrum. 9 (2014) C05063, http://dx.doi.org/10. 1088/1748-0221/9/05

[128] O. Jagutzki, A. Cerezo, A. Czasch, R. Dörner, M. Hattaß, V. Mergel, U. Spillmann, K. Ullmann-Pfleger, T. Weber, H. Schmidt-Böcking, G.D.W. Smith, IEEE-Trans. Nucl. Sci. 49 (2002) 2477.

[129] German Patent DE10335718 B4.

130] A. Oelsner, P. Lushchyk, D. Kutnyakhov, A. Fognini, D. Perriard, G. Salvatella, C. Stieger, R. Gort, T. Bähler, T. Michlmayer, W. Wurth, Y. Acremann, A. Vaterlaus, G. Schönhense, in preparation.

[131] W. Ackermann, G. Asova, V. Ayvazyan, A. Azima, N. Baboi, J. Bahr, V. Balandin, B. Beutner, A. Brandt, A. Bolzmann, Nat. Photon 1 (2007) 336. 